Universidade de SÃo Paulo

Faculdade de Filosofia, Ciências e Letras de Ribeirão Preto Pós-GraduaÇão em Física Aplicada à Medicina e Biologia

\title{
Zeros de Fisher e aspectos críticos do modelo de Ising dipolar
}

Jacyana Saraiva Marthes Fonseca

Ribeirão Preto - SP 


\section{JACYANA SARAIVA MARTHES FONSECA}

\section{Zeros de Fisher e aspectos críticos do modelo de Ising dipolar}

Dissertação apresentada à Faculdade de Filosofia, Ciências e Letras de Ribeirão Preto da Universidade de São Paulo, como parte das exigências para a obtenção do título de Mestre em Ciências

Área de Concentração:

Física Aplicada à Medicina e Biologia

Orientador:

Prof. Dr. Nelson Augusto Alves

Ribeirão Preto - SP 
AUTORIZO A REPRODUÇÃO E DIVULGAÇÃO TOTAL OU PARCIAL DESTE TRABALHO, POR QUALQUER MEIO CONVENCIONAL OU ELETRÔNICO, PARA FINS DE ESTUdO E PESQUISA, DESDE QUE CITADA A FONTE.

Fonseca, Jacyana Saraiva Marthes.

Zeros de Fisher e aspectos críticos do modelo de Ising dipolar/ Jacyana Saraiva Marthes Fonseca; orientador Prof. Dr. Nelson Augusto Alves. Ribeirão Preto, 2011. 127 p.

Dissertação (Mestrado - Programa de Pós-Graduação em Física Aplicada à Medicina e Biologia) - Faculdade de Filosofia, Ciências e Letras de Ribeirão Preto da Universidade de São Paulo.

1. transições de fase. 2. zeros complexos da função de partição. 3. algoritmo multicanônico. 4. modelo de Ising dipolar. 
Nome: FonseCA, Jacyana Saraiva Marthes

Título: Zeros de Fisher e aspectos críticos do modelo de Ising dipolar

Dissertação apresentada à Faculdade de Filosofia, Ciências e Letras de Ribeirão Preto da Universidade de São Paulo, como parte das exigências para a obtenção do título de Mestre em Ciências

Aprovado em:

\section{Banca Examinadora}

Prof. Dr. :

Julgamento:

Prof. Dr. :

Julgamento:

Prof. Dr. :

Julgamento:
Instituição:

Assinatura:

Instituição:

Assinatura:

Instituição:

Assinatura: 
Este trabalho é dedicado aos meus avós Kleib e Mariana. 


\section{AGRADECIMENTOS}

Gostaria neste agradecimento de lembrar das pessoas que contribuiram direta ou indiretamente para a realização deste trabalho. Agradeço em primeiro lugar ao meu orientador Nelson, pela idealização do projeto desta dissertação, por ter me recebido de braços abertos como sua orientanda, pela dedicação que tem ao seu trabalho e aos seus alunos, e principalmente pela paciência e compreensão que teve comigo diante dos problemas que venho enfrentando nestes últimos anos. Agradeço aos professores do Departamento de Física do Ibilce-UNESP, responsáveis pela minha formação interdisciplinar e pelo incentivo em seguir a carreira acadêmica, em especial aos professores doutores Jorge Chahine, que despertou em mim o interesse pelas simulações em física estatística, José Roberto Ruggiero, pelas aulas de física computacional e também pela sua coordenação no curso de Física Biológica, Elso Drigo Filho, pelas agradáveis aulas de física matemática e quântica, pelas orientações, carinho e amizade, e Marcelo Fossey, pelos quase três anos de trabalho juntos e pela amizade. Agradeço a todos os professores do programa FAMB, em especial aos professores doutores Alexandre, grande conselheiro, e Marcelo Mulato, pela ótima coordenação. Aos profs. Drs. Marco Antônio da Silva e Antônio Caliri, da FCFRP. Aos funcionários do Departamento de Física Nilza, Sônia, Ricardo, Fábio, dentre outros e ao pessoal da seção de pós, em especial Maria Inês e Denise. Agradeço com carinho aos meus colegas de laboratório, que acabaram por se tornar grandes amigos: Leandro, grande colaborador desse trabalho, pela ajuda e amizade, Rafael (saudades) pelas cervejadas e conversas de físico, Matheus, pela disponibilidade em me ouvir tagarelar nas horas de almoço e lanche sem reclamar e Guidolin, fundamental para a nossa sobrevivência diante dos problemas do Atlas. Ao meu namorado Francisco, pelo amor, paciência e resistência diante de meus 
afazeres intermináveis. Ao baiano arretado Raimundo, pela sua simpatia e alegria de sempre. A todos os amigos do programa FAMB: Sandro, Raul, Breno, Marcelo, Juliana, Fernanda, Aquino, Lindomar, Rodrigo, Denise, Diogo, Mirko, Tatiane, Michele, Marcelo, Érika, Ebenézer e todos os outros não citados. Ao meu amigão vô. Às minhas eternas amigas Ariadne, Talita, Sthefhany e Fernanda, às quais não são necessárias palavras. Aos grandes amigos que fiz ao morar na casa 13: Iraci, Isabel, Marco, Lourdes, Leila e Flávia. A todos os outros amigos da casa 13. Agradeço aos meus pais e às minhas irmãs, Mariana e Juliana, por sempre acreditarem em meu potencial. Às minhas tias Lígia, Marialba e Kátia, que sempre me ajudaram quando precisei. À reitoria e coordenadores dos cursos de física médica, ISE e engenharias da UNIFEB, em especial ao prof. Dr. Osvaldo Aiello, por me propiciarem a oportunidade de ingressar na carreira de docência e reconhecerem o meu trabalho. Agradeço à CAPES pela bolsa e à FAPESP pelo apoio ao grupo de pesquisa. 
Foi o tempo que dedicastes à tua rosa que fez tua rosa tão importante

Antoine de Saint-Exupéry 


\section{RESUMo}

FONSECA, J. S. M. Zeros de Fisher e aspectos críticos do modelo de Ising dipolar. 127 p. Dissertação (Mestrado) - Faculdade de Filosofia, Ciências e Letras de Ribeirão Preto, Universidade de São Paulo, Ribeirão Preto, 2011.

Estudamos o comportamento crítico do modelo de Ising com interação dipolar, em redes bidimensionais regulares. Este modelo apresenta um cenário fenomenologicamente rico devido ao efeito de frustração causado pela competição entre as interações de troca do Ising puro e a interação dipolar. A criticalidade do modelo foi estudada a partir das relações de escala de tamanho finito para os zeros da função de partição no plano complexo da temperatura. Esta abordagem nunca foi utilizada no estudo do modelo em questão. Nosso estudo se baseia em simulações de Monte Carlo usando o algoritmo multicanônico. O objetivo deste trabalho é obter a temperatura crítica em função do acoplamento $\delta$ (razão entre as intensidades dos acoplamentos ferromagnético e dipolar) e construir uma parte do diagrama de fase do modelo. Diferentes partes do diagrama de fase ainda não apresentam indicações conclusivas a respeito da ordem das linhas de transição. Em particular, há evidências na literatura de um ponto tricrítico para $\delta$ no intervalo [0.90,1.00], mas sua localização precisa não é conhecida. Nossas simulações indicam que o ponto tricrítico não se localiza no intervalo acima. Nossos resultados mostraram que, para $\delta \in[0.89,1.10]$, a fase do tipo faixas com $h=1$ passa para a fase tetragonal através de uma transição de segunda ordem. A análise de FSS para os zeros da função de partição na variável temperatura, apresenta, para $\delta=1.20$, uma transição de fase de segunda ordem e para $\delta=1.30$, uma transição de fase de primeira ordem. Dessa forma, o ponto tricrítico ocorre somente entre $\delta=1.20$ e 1.30. Realizamos um estudo 
complementar baseado na abordagem microcanônica e observamos duas transições de fase de segunda ordem para $\delta=1.20$ e duas transições de fase de primeira ordem para $\delta=1.30$, que indica a presença da fase nemática intermediária. 


\section{Abstract}

FONSECA, J. S. M. Fisher's zeros and critical aspects of the dipolar Ising model. 127 p. Dissertation (M.Sc.) - Faculdade de Filosofia, Ciências e Letras de Ribeirão Preto, Universidade de São Paulo, Ribeirão Preto, 2011.

We study the critical behavior of the dipolar Ising model on two-dimensional regular lattices. This model presents a phenomenologically rich scenario due to the effect of frustration caused by the competition between the pure Ising interaction and the dipolar one. To study the criticality of this model we apply finite size scaling relations for the partition function zeros in the complex temperature plane. The partition function zeros analysis has never been used before to study such model with long-range interactions. Our study relies on Monte Carlo simulations using the multicanonical algorithm. Our goal is to obtain the critical temperature as a function of the coupling $\delta$ (the ratio between the ferromagnetic and dipolar couplings) to construct a part of the phase diagram. Different parts of the phase diagram do not present a conclusive results about the order of the phase transition lines. In particular, there is evidence of a tricritical point for $\delta \in[0.90,1.00]$, but its precise location is unknown. Our simulations indicate that the tricritical point is not located in the above range. Our FSS analysis show that for $\delta=1.20$ the striped-tetragonal transition is a second-order phase transition and for $\delta=1.30$ it is a first-order one. Thus, the tricritical point must occur between $\delta=1.2$ and $\delta=1.3$. We have used a microcanonical approach to study the criticality of this model too. This approach indicates two second-order phase transitions for $\delta=1.20$ and two first-order phase transitions for $\delta=1.30$. Therefore, it presents evidences for the presence of an intermediate nematic phase. 


\section{LISTA DE FigURAS}

1.1 Fase do tipo faixas com largura $h=4 \ldots \ldots \ldots 5$

1.2 Diferentes fases observadas em filmes finos de $\mathrm{Fe}$ em $\mathrm{Cu}$ pela técnica SEMPA (microscopia eletrônica de varredura com análise de poLarização). . . . . . . . . . . . . . . . 6

1.3 Configurações dos spins para uma rede de largura $L=56$ obtidas por simulações de Monte Carlo. . . . . . . . . . . . . . . . 7

1.4 Histogramas da densidade de energia $e_{s}$ obtidos de uma simulação multicanônica juntamente com os histogramas repesados para diferentes temperaturas no ensemble canônico para o modelo de Ising bidimensional em uma rede $20 \times 20 \ldots \ldots$. . . . . . . 9

2.1 (a) Diagrama PT para um sistema fluido. (b) Diagrama HT para um sistema magnético simples. . . . . . . . . . . . . . 13

2.2 (a) Diagrama $\rho T$ para um sistema fluido puro. (b) Diagrama $M T$ para um sistema magnético simples. . . . . . . . . . . . . 14

2.3 Medidas da curva de coexistência para fluidos. . . . . . . . . . . 15

2.4 Ilustração de uma mudança de escala de uma função $f(\mathbf{r})$ para uma função $f(\lambda \mathbf{r}) \ldots \ldots \ldots \ldots \ldots$. . . . . . . . . . . . . . . . . . . . .

2.5 Comportamento do comprimento de correlação $\xi$ para diferentes temperaturas. ........................ . . 21 
3.1 Comportamento analítico a uma dada temperatura das funções termodinâmicas para: (a) um sistema em uma única fase, (b) um sistema que passa por duas transições de fase. . . . . . . . . . . . . 27

3.2 Ilustração da distribuição de zeros da função de partição próximos ao eixo real positivo. . . . . . . . . . . . . . . . 30

3.3 Ilustração de um pequeno pedaço da rede de spins para o modelo de Ising 1D . . . . . . . . . . . . . . . . . . 31

4.1 Configurações de spin nas temperaturas de transição para uma rede $L=56$ e $\delta=2$. (a) fase do tipo faixas, (b) sistema na temperatura de transição da fase de faixas para a fase nemática, (c) fase nemática, (d) sistema na temperatura de transição da fase nemática para o líquido tetragonal, (e) líquido tetragonal. . . . . . . . . . . . . . 40

4.2 Representação esquemática de (a) uma deslocação, (b) e (c) tipos de disclinações que podem ocorrer em fases do tipo faixas. . . . . . . . . 41

4.3 Diagrama de fase em função do acoplamento $\delta$ para o modelo de Ising dipolar. .......................... 42

4.4 Diagrama de fase em função do acoplamento $\delta$ obtido por cálculo de campo médio. . . . . . . . . . . . . . . . . . 4 42

6.1 Representação das condições periódicas de contorno. . . . . . . . . . 55

6.2 Energias calculadas para os estados do tipo faixas $h=1,2$ e $3 \mathrm{em}$ função de $\delta$ para $L=128$. . . . . . . . . . . . . . . 57

6.3 Histogramas de energia produzidos em diferentes repetições $n$ para atualização dos parâmetros multicanônicos $a^{n}(E)$ e $b^{n}(E)$ para $\delta=0.89 .60$

6.4 Parâmetros multicanônicos $a(E)$ finais utilizados para a produção de dados para $L=48 \ldots \ldots \ldots$. . . . . . . . . . . . 61

6.5 Escaneamento dos zeros da função de partição canônica em $\beta$ para

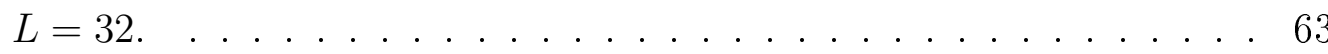


6.6 Zeros em $u=e^{-\beta}$ para $L=32$ obtidos de uma das 16 séries produzidas para (a) $(\delta=0.89)$ todos os zeros obtidos, (b) $(\delta=0.89)$ ampliação da região de interesse, (c) $(\delta=1.30)$ todos os zeros obtidos, (d) $(\delta=1.30)$ ampliação da região de interesse. . . . . . . . . 64

7.1 Regressão linear para $-\ln \operatorname{Im}\left[u_{1}^{0}(L)\right]$ versus $\ln L$ com os respectivos valores de $d \nu$ para diferentes valores de $\delta \ldots \ldots \ldots$. . . . . 67

7.2 Regressão linear para $-\ln \operatorname{Im}\left[u_{1}^{0}(L)\right]$ versus $\ln L$ com os respectivos valores de $d \nu$ para diferentes valores de $\delta \ldots \ldots . \ldots 68$

7.3 Regressão linear para $\operatorname{Re} \beta(L)$ versus $L^{-1 / \nu}$ com os respectivos valores

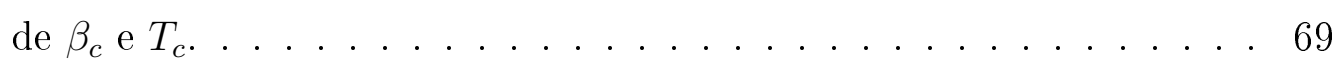

7.4 Regressão linear para $\operatorname{Re} \beta(L)$ versus $L^{-1 / \nu}$ com os respectivos valores

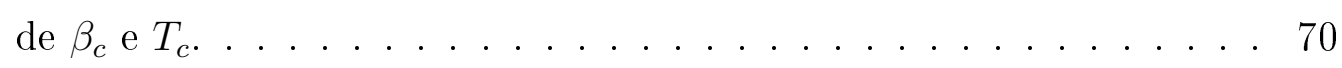

7.5 Diagrama de fase de $T_{c}$ versus $\delta$ construído a partir de nossos resultados. 71

7.6 Diagrama de fase de $T_{c}$ versus $\delta$ retirado da ref.(11) . . . . . . . 71

A.1 Parâmetros multicanônicos $a(E)$ finais utilizados para a produção de dados para $\delta=0.89 \ldots \ldots \ldots \ldots \ldots$

A.2 Parâmetros multicanônicos $b(E)$ finais utilizados para a produção de dados para $\delta=0.89 \ldots \ldots \ldots \ldots$. . . . . . . . . 84

A.3 Parâmetros multicanônicos $a(E)$ finais utilizados para a produção de dados para $\delta=0.91$.

A.4 Parâmetros multicanônicos $b(E)$ finais utilizados para a produção de dados para $\delta=0.91 \ldots \ldots \ldots \ldots \ldots$

A.5 Parâmetros multicanônicos $a(E)$ finais utilizados para a produção de dados para $\delta=0.93 \ldots \ldots \ldots \ldots$. . . . . . . . . 87

A.6 Parâmetros multicanônicos $b(E)$ finais utilizados para a produção de

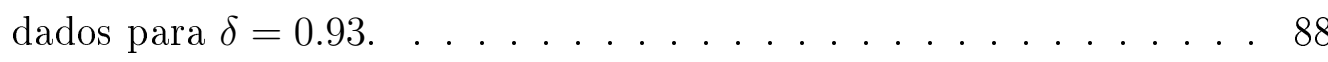


A.7 Parâmetros multicanônicos $a(E)$ finais utilizados para a produção de dados para $\delta=0.95 \ldots \ldots \ldots \ldots$. . . . . . . . . . . . . . . . . .

A.8 Parâmetros multicanônicos $b(E)$ finais utilizados para a produção de dados para $\delta=0.95 \ldots \ldots \ldots \ldots$. . . . . . . . . . . . . . . .

A.9 Parâmetros multicanônicos $a(E)$ finais utilizados para a produção de dados para $\delta=0.97$.

A.10 Parâmetros multicanônicos $b(E)$ finais utilizados para a produção de dados para $\delta=0.97$.

A.11 Parâmetros multicanônicos $a(E)$ finais utilizados para a produção de dados para $\delta=1.00$.

A.12 Parâmetros multicanônicos $b(E)$ finais utilizados para a produção de dados para $\delta=1.00$.

A.13 Parâmetros multicanônicos $a(E)$ finais utilizados para a produção de dados para $\delta=1.10 \ldots \ldots \ldots \ldots . \ldots \ldots$

A.14 Parâmetros multicanônicos $b(E)$ finais utilizados para a produção de dados para $\delta=1.10 \ldots \ldots \ldots \ldots$. . . . . . . . . . . . . .

A.15 Parâmetros multicanônicos $a(E)$ finais utilizados para a produção de dados para $\delta=1.20 \ldots \ldots \ldots \ldots$. . . . . . . . . . . . . . . . . . .

A.16 Parâmetros multicanônicos $b(E)$ finais utilizados para a produção de dados para $\delta=1.20$.

A.17 Parâmetros multicanônicos $a(E)$ finais utilizados para a produção de dados para $\delta=1.30$.

A.18 Parâmetros multicanônicos $b(E)$ finais utilizados para a produção de dados para $\delta=1.30$.

C.1 Calores específicos microcanônicos para (a) $\delta=0.89$ (b) delta $=0.91$, (c) $\delta=0.93,($ d) $\delta=0.95 \ldots \ldots \ldots$. . . . . . . . . . . . . . . . . . 
C.2 Calores específicos microcanônicos para (a) $\delta=0.97$ (b) delta $=1.00$,

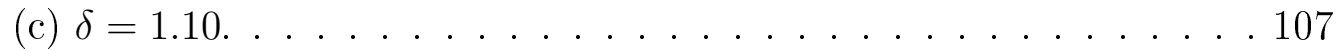

C.3 $\delta=1.20($ a) $b(E)$ versus $E / N$, (b) Calor específico microcanônico. . . 108

C.4 $\delta=1.30($ a) $b(E)$ versus $E / N$, (b) calor específico microcanônico. . 109 


\section{LISTA DE TABELAS}

2.1 Definições de alguns expoentes críticos para sistemas fluido e magnético. 16

2.2 Algumas igualdades entre expoentes críticos. . . . . . . . . . . . . 19

4.1 Dependência da largura das faixas $h$ com acoplamento $\delta \ldots . . . . .39$

4.2 Ordem de transição da fase do tipo faixas para a fase tetragonal obtidas na ref. (12) para diferentes valores de $\delta \ldots . . . . . . .43$

4.3 Resumo das transições de fase observadas para alguns acoplamentos $\delta$ conforme obtidas em diferentes trabalhos. . . . . . . . . . . 43

6.1 Relação entre $\delta, h$ e energia do estado fundamental utilizada nas

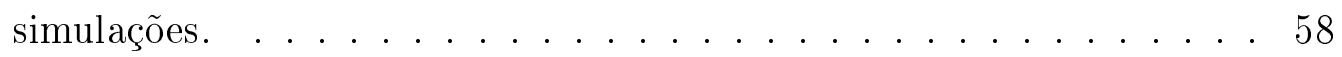

6.2 Histograma para baixas energias com $L=12$ para $\epsilon=0.5$ e $\epsilon=1$. . . 58

$6.3 n_{M C}$ utilizados na produção de dados. . . . . . . . . . . . . 61

7.1 Resultados obtidos a partir da FSS para os zeros da função de partição. 66

7.2 Temperaturas críticas obtidas a partir da FSS para $\operatorname{Re}(\beta) . \ldots . . \quad 72$

7.3 Comparação entre $b_{c}(E)$ e $\beta_{c}$ para $\delta=1.20$ e $1.30 \ldots \ldots . . . . .74$

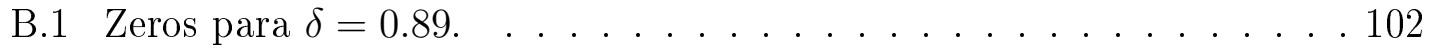

B.2 Zeros para $\delta=0.91 \ldots \ldots \ldots \ldots \ldots \ldots \ldots \ldots$

B.3 Zeros para $\delta=0.93 \ldots \ldots \ldots \ldots \ldots$. . . . . . . . . . . . . . . 
xviii

B.4 Zeros para $\delta=0.95 \ldots \ldots \ldots \ldots \ldots \ldots \ldots$

B.5 Zeros para $\delta=0.97 \ldots \ldots \ldots \ldots$. . . . . . . . . . . . . . . .

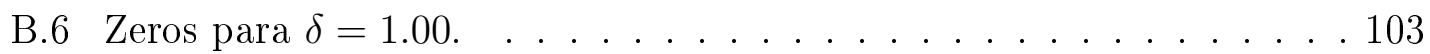

B.7 Zeros para $\delta=1.10 . \ldots \ldots \ldots \ldots \ldots$. . . . . . . . . . . . . . .

B.8 Zeros para $\delta=1.20 \ldots \ldots \ldots \ldots \ldots$. . . . . . . . . . . . . . .

B.9 Zeros para $\delta=1.30 . \ldots \ldots \ldots \ldots$. . . . . . . . . . . . . . . 


\section{SUMÁRIO}

Lista de Figuras $\quad$ xii

Lista de Tabelas $\quad$ xvii

1 Introdução 4

2 Transições de fase $\quad 11$

2.1 Introdução . . . . . . . . . . . . . . . . . . . . . . . . 11

2.2 Os expoentes críticos . . . . . . . . . . . . . . . . 14

2.2.1 Relações entre os expoentes críticos . . . . . . . . . . . 17

2.3 Análise de escala de tamanho finito . . . . . . . . . . . . . 19

3 Zeros da função de partição 22

3.1 Introdução . . . . . . . . . . . . . . . . . . 22

3.2 Zeros da função de partição . . . . . . . . . . . . . . . . . . 24

3.2.1 Zeros de Yang-Lee . . . . . . . . . . . . . . . 24

3.2 .2 Zeros de Fisher . . . . . . . . . . . . . 28

3.3 Escala de tamanho finito para os zeros da função de partição . . . . . 31

3.3.1 Grupo de renormalização . . . . . . . . . . . . . . 31

3.3.2 Escala de tamanho finito . . . . . . . . . . . 33 
4 O modelo de Ising dipolar bidimensional

4.1 Introdução . . . . . . . . . . . . . . . . . . 36

4.2 A hamiltoniana do modelo . . . . . . . . . . . . . . 36

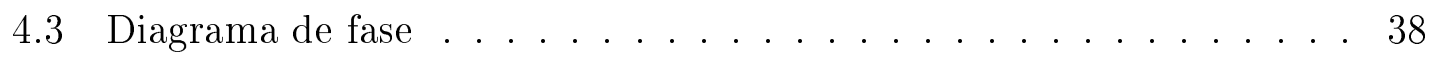

5 Métodos de Monte Carlo $\quad 44$

5.1 Introdução . . . . . . . . . . . . . . . . . . . . 44

5.2 Ensembles generalizados . . . . . . . . . . . . . . 46

5.2 .1 O algoritmo entrópico . . . . . . . . . . 47

5.2 .2 O algoritmo multicanônico . . . . . . . . . . 48

5.2.2.1 Estimativas canônicas . . . . . . . . . . 48

5.2.2.2 Relações de recorrência para obtenção do peso multicanônico ................ 50

6 Simulação do modelo

6.1 Condições periódicas de contorno e o método da soma de Ewald . . . 54

6.2 Simulações multicanônicas . . . . . . . . . . . . . . 56

6.3 Zeros da função de partição canônica . . . . . . . . . . . . . . . . . 61

$\begin{array}{lll}7 & \text { Resultados e discussão } & 65\end{array}$

7.1 Análise de escala de tamanho finito para os zeros da função de partição 66

7.2 Possibilidade de outra transição de fase . . . . . . . . . . . . . . 73

8 Conclusões 
A Parâmetros Multicanônicos

B Zeros complexos da função de partição

C Análise microcanônica 


\section{CApÍtulo}

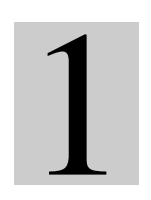

\section{INTRODUÇÃO}

Entende-se por filme fino uma camada de material que pode ter desde frações de nanômetro até vários micrômetros de espessura. As aplicações deste tipo de filme são vastas, e dentre elas estão a fabricação de componentes eletrônicos semicondutores, revestimentos ópticos e memórias de computadores. Filmes de metal sobre metal são utilizados na eletrônica, armazenamento de dados e catálises. Filmes moleculares têm sido aplicados na biotecnologia e farmacologia. Filmes magnéticos ultrafinos, que têm espessuras próximas a uma camada atômica, têm sido largamente estudados. Como exemplo podemos citar dois tipos: filmes de metal sobre metal, como ferro em cobre (Fe/Co) (1) e cobre em ouro (Co/Au) (2), e filmes de cerâmicas supercondutoras como o R-Ba-Cu, onde R é terra-rara da série dos lantanídeos. As mudanças, tanto no conteúdo de oxigênio como na composição dessas cerâmicas, têm um papel importante na definição de seu comportamento supercondutor, isolante ou magnético (3). A produção e utilização de filmes ultrafinos têm sido possível graças ao avanço na manipulação de materiais em escala atômica (nanotecnologia), com técnicas de crescimento e de caracterização cada vez melhores. Embora haja um grande interesse na aplicação tecnológica, o estudo teórico desses filmes permite uma melhor compreensão das interações atômicas. Essa compreensão é sem dúvida de extrema importância. O desenvolvimento de materiais para uso específico requer um entendimento detalhado das interações microscópicas e de como essas são afetadas pela composição e preparação, resultando nas propriedades do material. O uso de filmes magnéticos para armazenamento de dados, por exemplo, exige que a magnetização do filme seja definida e lida com alto grau de acurácia e resolução 


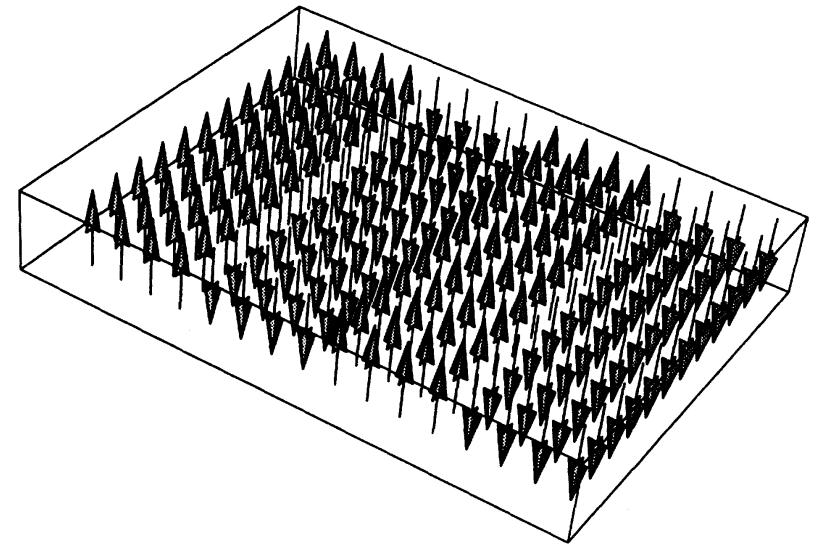

Figura 1.1: Fase do tipo faixas com largura $h=4$. Figura retirada de (4).

espacial (1).

Filmes magnéticos ultrafinos apresentam propriedades físicas não usuais, por exemplo, a existência de uma temperatura acima da qual a magnetização ocorre na direção do plano do filme, e abaixo desta, perpendicular ao plano. Para o intervalo de temperatura em que ocorre a magnetização perpendicular, são observadas fases com domínios tipo faixas, onde o sistema é constituído por faixas alternadas ferromagnéticas (veja a figura 1.1), na forma de labirintos, dentre outras. Essas fases, apesar de apresentarem magnetização total praticamente nula, apresentam um alto grau de ordenamento.

A figura 1.2 ilustra as diferentes fases observadas em filmes de $\mathrm{Fe}$ em $\mathrm{Cu}$ pela técnica SEMPA (microscopia eletrônica de varredura com análise de polarização). As figuras $1.2 \mathrm{a}$ - 1.2d apresentam o padrão de evolução dos domínios magnéticos à temperatura ambiente conforme se diminui a espessura do filme. Nas figuras $1.2 \mathrm{e}-1.2 \mathrm{~h}$, temos o comportamento do filme conforme a temperatura é aumentada. Observe que a diminuição da espessura corresponde ao aumento da temperatura do filme. Veja que para baixas temperaturas temos aproximadamente uma fase do tipo faixas. Se a temperatura for aumentada, o sistema passa para uma fase do tipo labirinto até chegar a uma fase caracterizada por faixas e bolhas. A altas temperaturas encontramos a fase paramagnética (figura 1.2h). Esse tipo de sistema tem sido estudado teoricamente utilizando-se a aproximação em que os filmes 


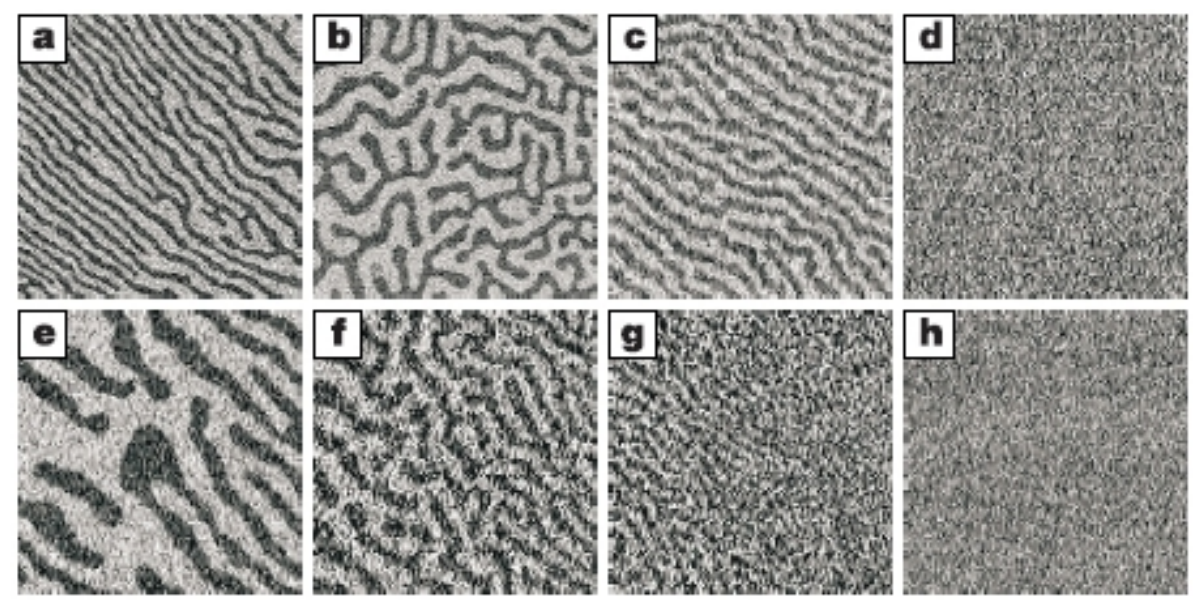

Figura 1.2: Diferentes fases observadas em filmes finos de $\mathrm{Fe}$ em $\mathrm{Cu}$ pela técnica SEMPA (microscopia eletrônica de varredura com análise de polarização). Figura retirada de $(5)$

são constituídos de uma única camada, representado por um modelo de Ising que considera, além da interação de troca entre primeiros vizinhos, a interação dipolar entre todos os spins. O efeito conjunto da interação dipolar de longo alcance com a interação de troca de curto alcance conduz ao fenômeno de frustração e explica a origem de uma variedade de interessantes e incomuns fenômenos magnéticos. A hamiltoniana utilizada para representar as interações entre spins nesse sistema é dada por

$$
E(\sigma)=-\delta \sum_{<i, j>} \sigma_{i} \sigma_{j}+\sum_{i \neq j} \frac{\sigma_{i} \sigma_{j}}{r_{i j}^{3}}
$$

onde $\delta=J / g$ é a razão entre os acoplamentos das interações de troca $(J>0)$ e dipolar $(g>0)$. O primeiro termo representa o termo de Ising simples, que leva em conta somente a interação entre primeiros vizinhos. O segundo termo representa a interação dipolar e leva em conta a interação entre todos os pares de spins. O método da soma de Ewald $(6,7)$, desenvolvido para lidar com interações de longo alcance, já tem sido utilizado por nosso grupo de pesquisa (8) na implementação das condições periódicas de contorno para as interações dipolares. A figura 1.3 ilustra as três fases observadas para diferentes temperaturas em simulações de Monte Carlo usando $\delta=2$. Nesta figura temos as fases do tipo faixas, nemática e tetragonal. Vários autores têm trabalhado na tentativa de caracterizar o diagrama de fase do modelo como função do acoplamento $\delta(9,10,11,12,13)$. Entretanto, muitas 


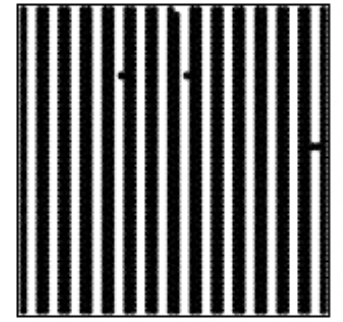

(a) $T=0.710$

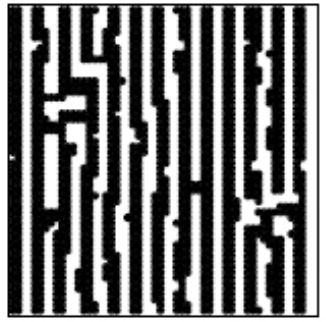

(b) $T=0.790$

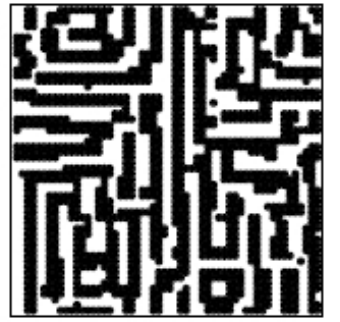

(c) $T=0.840$

Figura 1.3: Configurações dos spins para uma rede de largura $L=56$ obtidas por simulações de Monte Carlo em temperaturas que apresentam: (a) fase tipo faixas, (b) nemática e (c) tetragonal. Figura retirada da ref. (13).

partes do diagrama de fase ainda não apresentam indicações conclusivas da ordem das transições, ou seja, o diagrama de fase do modelo ainda não é completamente conhecido. Em particular, a existência de um ponto tricrítico entre $\delta=0.89$ e $\delta=1$ é prevista por métodos de Monte Carlo (11), mas sua localização precisa ainda não é conhecida. Neste trabalho, pretendemos investigar a existência desse ponto tricrítico e assim, definir essa parte do diagrama de fase.

Divergências entre as predições por métodos de Monte Carlo e cálculos de Campo Médio colocam em dúvida a existência da fase intermediária nemática, existente entre a fase do tipo faixas e a fase tetragonal, para o intervalo do acoplamento $\delta$ no qual estamos trabalhando $(\delta \in[0.89-1.20])$. Os zeros complexos da função de partição permitem caracterizar a criticalidade de um sistema e, porisso, lançamos mão do seu uso na busca da localização do ponto tricrítico e assim poder caracterizar o diagrama de fase na região de interesse. Vale salientar que o modelo em questão ainda não foi estudado via zeros da função de partição.

O comportamento de escala de tamanho finito dos zeros da função de partição tem sido largamente utilizado no estudo da criticalidade dos modelos de Ising $(14,15,16,17,18,19)$ e Potts $(20)$. O estudo dos zeros da função de partição teve sua origem em 1952 com o trabalho de Yang e Lee (21). Estes autores aplicaram o conceito dos zeros da função de partição grande canônica no estudo do modelo de gás monoatômico e mostraram que, apesar de somente valores reais da fugacidade apresentarem interesse físico, o comportamento analítico das funções 
termodinâmicas pode ser revelado se olharmos para o plano complexo. Com esse enfoque, puderam obter uma descrição das fases condensadas do gás e das regiões de transição no limite termodinâmico. No mesmo trabalho foi desenvolvido o Teorema do Círculo. Este teorema diz que os zeros, no plano complexo do campo magnético, externo ocorrem em um círculo unitário para o modelo de Ising ferromagnético. Enquanto Yang e Lee consideraram os zeros no plano complexo do campo magnético para sistemas de spins, Fisher (18) em 1965 chamou atenção para a relevância dos zeros da função de partição no plano complexo da temperatura. Usando o modelo de Ising para redes bidimensionais com geometria quadrada como exemplo, ele mostrou que os zeros da função de partição eram distribuídos em duas curvas fechadas no plano complexo no limite termodinâmico e que a singularidade logarítmica do modelo bidimensional derivava da distribuição desses zeros. Entretanto, não temos um teorema do círculo para os zeros de Fisher, dado que o comportamento desses zeros depende fortemente dos detalhes do sistema. A ausência de um resultado geral para os zeros de Fisher se deve ao fato de que a temperatura crítica é uma quantidade que varia de um sistema para outro (22). Independente do modelo a ser estudado, sabemos que a distribuição da densidade de zeros próximo ao eixo real positivo da temperatura determina o comportamento crítico. Essa abordagem tem sido bastante explorada em inúmeros sistemas termodinâmicos, em particular, para determinar a temperatura e os expoentes críticos.

Para obter a função de partição é necessário que se conheça a densidade de estados do sistema. A densidade de estados do modelo de Ising bidimensional dipolar será obtida por simulações de Monte Carlo em vista da impossibilidade de uma abordagem analítica. Com a abstração via um modelo hamiltoniano, todas as propriedades do sistema físico podem ser determinadas por meio das simulações numéricas com a vantagem de se poder isolar diferentes efeitos físicos existentes no modelo. Dessa forma, simulações numéricas permitem também um maior controle de aspectos dificilmente conseguidos em laboratório e assim, esclarecer os principais fatores que levam aos fenômenos observados. Utilizaremos o algoritmo multicanônico nas simulações de Monte Carlo. O algoritmo multicanônico, proposto por Berg e Neuhaus $(23,24,25)$, foi desenvolvido com o intuito de contornar o problema das barreiras de energia livre encontradas em transições de primeira ordem. $\mathrm{O}$ 


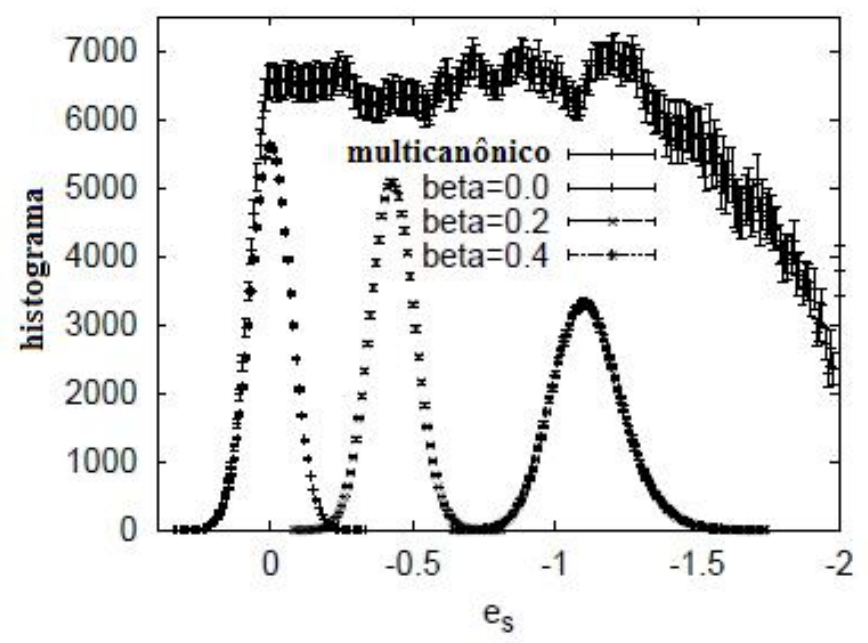

Figura 1.4: Histogramas de energia obtidos de uma simulação multicanônica juntamente com os histogramas repesados para diferentes temperaturas no ensemble canônico para o modelo de Ising $2 \mathrm{~d}$ em uma rede $20 \times 20$. Figura adaptada de (26).

algoritmo multicanônico caracteriza-se por procurar amostrar todas as energias com a mesma probabilidade fazendo com que o histograma obtido seja aproximadamente uniforme. Desta maneira, configurações importantes mas raras no ensemble canônico são amostradas, permitindo assim que barreiras de energia livre sejam ultrapassadas. Outra vantagem do algoritmo multicanônico é que, enquanto as simulações de Monte Carlo no ensemble canônico são efetuadas a uma temperatura fixa e só podem ser extrapoladas pela técnica de repesagem para temperaturas bem próximas à temperatura simulada, as simulações no ensemble multicanônico permitem o cálculo de valores esperados canônicos em qualquer temperatura. A figura 1.4 apresenta os histogramas de densidade de energia $e_{s}(E / N$ - energia total dividida pelo número de partículas do sistema) obtidos para o modelo de Ising bidimensional em uma rede quadrada $20 \times 20$ para uma simulação multicanônica. O histograma aproximadamente uniforme, que abrange um maior intervalo de energia, é o obtido diretamente das simulações multicanônicas e os outros três histogramas são os histogramas repesados para diferentes valores de $\beta=1 / k_{B} T$, dado em unidades de $k_{B}$ onde $T$ é a temperatura do sistema.

Quando falamos em simulações de Monte Carlo, trabalhamos com um número de spins finito e bem distante do limite termodinâmico e esse efeito deve ser 
considerado. Esses efeitos são particularmente importantes nas regiões críticas. Se variarmos o tamanho do sistema nas simulações, podemos utilizar a teoria de escala de tamanho finito (Finite Size Scaling - FSS). Esta teoria é utilizada em simulações de Monte Carlo para estimar os expoentes críticos nas grandezas termodinâmicas como calor específico, por exemplo. No ponto crítico, a taxa de variação das funções termodinâmicas diverge e assume a forma tipo lei de potência com expoentes, chamados de expoentes críticos, que dependem apenas de algumas características do sistema. No presente trabalho utilizaremos relações de escala para os zeros da função de partição no plano complexo da temperatura com o intuito de obtermos a ordem da transição de fase e a temperatura crítica em função de vários valores do acoplamento $\delta$.

O processo de produção dos parâmetros multicanônicos, conforme veremos no capitulo 5.2.2, fornece informações correspondentes ao ensemble microcanônico. A partir da análise do comportamento da entropia microcanônica, podemos obter o calor específico do sistema em função da energia e, a partir deste, inferir a ordem da transição de fase. Esta análise será utilizada com o intuito de complementar a abordagem via zeros da função de partição, objeto principal dessa dissertação. 


\section{Capítulo}

\section{TRANSIÇÕES DE FASE}

\subsection{Introdução}

Neste capítulo veremos a definição de transições de fase de primeira e segunda ordem e a relação com os expoentes críticos. Todo o desenvolvimento deste capítulo foi baseado na referência (27). Veremos também que, apesar das simulações serem efetuadas em sistemas finitos, podemos obter os expoentes críticos e a ordem da transição de fase esperados no limite termodinâmico a partir da análise de FSS. Com relação à ordem de uma transição de fase, consideremos o critério de classificação de Ehrenfest e tomemos como exemplo a energia livre de Helmholtz F. Se sua primeira derivada é descontínua, temos uma transição de fase de primeira ordem. Se a primeira derivada for contínua, mas a segunda for descontínua, temos uma transição de segunda ordem, e assim sucessivamente. Uma transição de fase de segunda ordem, também chamada de transição contínua, corresponde a um ponto crítico no diagrama de fase. Para uma transição de primeira ordem temos geralmente uma descontinuidade na entropia, ou seja, uma descontinuidade na primeira derivada do potencial termodinâmico,

$$
S=\left(\frac{\partial F}{\partial T}\right)
$$

Uma das formas de se obter a ordem da transição de fase é observar o comportamento do parâmetro de ordem do sistema. Um parâmetro de ordem é uma variável extensiva que permite distinguir as diferentes fases do sistema e por conveniência, geralmente é escolhido de forma que seja nulo na fase desordenada. 
Considerando $F(H, T)$ para um sistema magnético, o parâmetro de ordem em relação ao campo magnético aplicado $H$ é dado por

$$
\phi=-\left(\frac{\partial F}{\partial H}\right),
$$

e o parâmetro de ordem em relação a temperatura $T$ é a entropia $S$ (dada anteriomente pela equação 2.1). Se o parâmetro de ordem for descontínuo em relação à variável independente (consideremos como exemplo a variável $T$ ), temos uma transição de primeira ordem. Em uma transição de segunda ordem, o parâmetro de ordem tem singularidades que obedecem relações tipo lei de potência,

$$
\phi \propto\left|\frac{T_{c}-T}{T_{c}}\right|^{\beta},
$$

assim como o calor específico e a susceptibilidade magnética,

$$
\begin{gathered}
c_{H}=-T\left(\frac{\partial^{2} F}{\partial T^{2}}\right)_{H} \propto\left|\frac{T_{c}-T}{T_{c}}\right|^{-\alpha}, \\
\chi=-\left(\frac{\partial^{2} F}{\partial H^{2}}\right)_{T} \propto\left|\frac{T_{c}-T}{T_{c}}\right|^{-\gamma},
\end{gathered}
$$

onde $\beta, \alpha$ e $\gamma$ são os chamados expoentes críticos.

Para ilustrar com maior clareza transições de fase de primeira e segunda ordem, utilizaremos como exemplo dois tipos de sistema: um sistema fluido e um sistema magnético simples. Para o sistema fluido, a equação de estado $f(P, \rho, T)=0$, relacionando a pressão $P$, densidade $\rho$ e a temperatura $T$, define uma superfície em três dimensões correspondente a estados de equilíbrio. Analogamente para um sistema magnético, temos uma equação de estado $g(H, M, T)$ relacionada ao campo magnético $H$, à magnetização $M$ e à temperatura $T$. Vamos considerar agora as projeções das superfícies $P \rho T$ e $H M T$ nos planos $P T$ e $H T$, respectivamente para o sistema fluido e para o sistema magnético (figura 2.1). Para o fluido observamos três regiões separadas correspondentes às fases sólida, líquida e gasosa. As fases sólida e gasosa se equilibram ao longo da curva de sublimação, as fases líquida e gasosa se equilibram ao longo da curva de pressão de vapor e as fases sólida e líquida ao longo da curva de fusão. No ponto triplo os três estados coexistem em equilíbrio. A curva de pressão de vapor termina em um ponto denominado ponto 


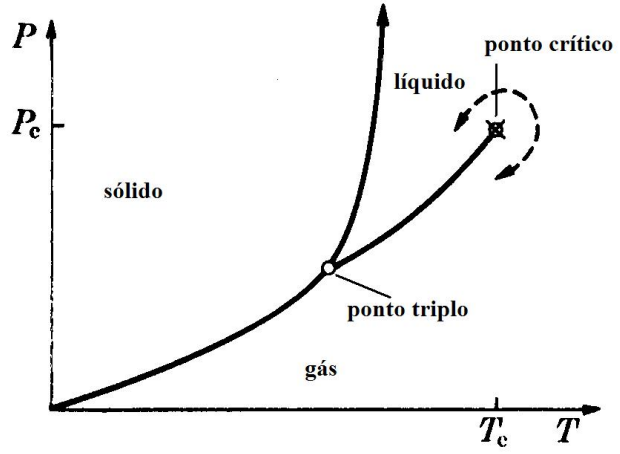

(a)

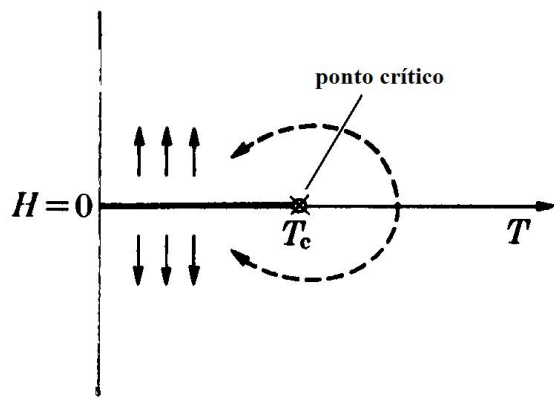

(b)

Figura 2.1: (a) Diagrama PT para um sistema fluido. (b) Diagrama HT para um sistema magnético simples. Figura adaptada de (27).

crítico, dado pelas coordenadas $P_{c}, T_{c}$ e $\rho_{c}$. A partir desse ponto não há diferença fundamental entre as fases líquida e gasosa. No caso magnético, observamos que existem duas fases: a ferromagnética e a antiferromagnética que estão em equilíbrio em uma curva quando $H=0$. Da mesma forma que no sistema fluido, a curva de transição termina no ponto crítico associado às coordenadas $H_{c}, T_{c}$ e $M_{c}$, de modo que a partir de tal ponto não há distinção entre as duas fases. Olhando agora para os planos $\rho T$ e $M T$ (figura 2.2), temos para o sistema fluido uma diferença significativa entre as densidades do líquido $\left(\rho_{L}\right)$ e do gás $\left(\rho_{G}\right)$ para baixas temperaturas. Conforme a temperatura aumenta e se aproxima de $T_{c}$, menor é a diferença entre $\rho_{L}-\rho_{G}$. Analogamente, para o sistema magnético temos que, conforme a temperatura aumenta para próximo de $T_{c}$, a magnetização se aproxima de 0 . Note que $\rho_{L}-\rho_{G}$ e $M$ são os parâmetros de ordem para os sistemas fluido e magnético, respectivamente. Veja que a termodinâmica descreve as propriedades do sistema nas linhas de mudança de fase de primeira ordem (no caso fluido, da fase sólida para a líquida, por exemplo). Entretanto, as propriedades dos sistemas no ponto crítico não são descritas pela termodinâmica. O comportamento de um ponto crítico é determinado pelas flutuações e requer, por isso mesmo, uma descrição microscópica, possível com o uso da Física Estatística. A Física Estatística permite que busquemos a compreensão das transições de fase e dos fenômenos críticos com a utilização de dois conceitos: universalidade e invariância de escala. A universalidade é definida pelo conjunto de expoentes críticos idênticos associados às transições 

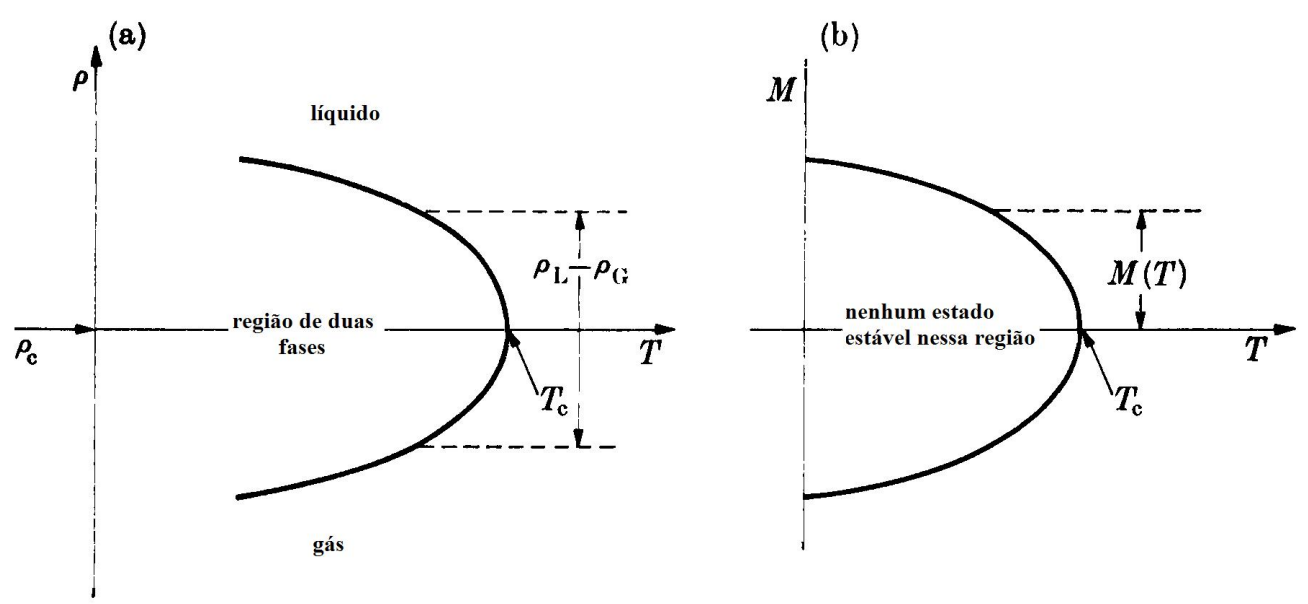

Figura 2.2: (a) Diagrama $\rho T$ para um sistema fluido puro. (b) Diagrama $M T$ para um sistema magnético simples. Figura adaptada de (27).

contínuas de sistemas diferentes. Dizemos que dois sistemas pertencem à mesma classe de universalidade se fornecem o mesmo conjunto de expoentes. Veremos na próxima seção a origem dos expoentes críticos.

\subsection{Os expoentes críticos}

Os estudos dos expoentes críticos iniciaram-se por volta de 1960 com Heller e Benedeck, Jacrot e outros. Até 1945, acreditava-se que a curva de coexistência de um sistema fluido era parabólica. Havia também uma predição de que para sistemas magnéticos $M^{2} \propto\left(T_{c}-T\right)$. Entretanto, as medidas de Heller e Benedeck mostraram que $M^{3} \propto\left(T_{c}-T\right)$ e as medidas de Guggenheim mostraram que $\frac{T^{3}}{T_{c}} \propto \frac{\rho}{\rho_{c}}$ (figura 2.3) para vários gases. Consideremos o caso magnético, sendo $M$ o parâmetro de ordem, este varia com $(-\epsilon)^{\beta}$, onde $\epsilon \equiv \frac{T-T_{c}}{T_{c}}$. Dessa forma, $\beta=1 / 3$ é o expoente crítico associado à magnetização do sistema. Se uma relação da forma $M=$ $B(-\epsilon)^{\beta}$ é válida, então algumas medidas próximas ao ponto crítico são suficientes para determinar o valor de $\beta$. A partir de então, os expoentes críticos começaram a ser utilizados para descrever o comportamento de várias quantidades de interesse próximo ao ponto crítico.

Consideremos uma função geral $f(\epsilon)$ positiva para exemplificar a origem de um 


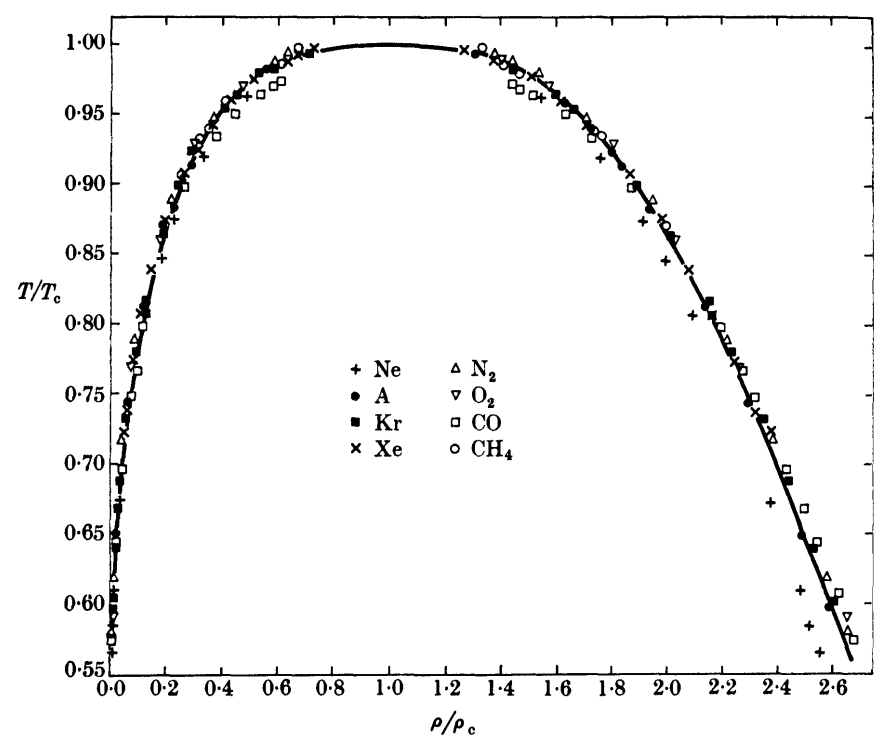

Figura 2.3: Medidas da curva de coexistência para fluidos. A curva sólida corresponde a um ajuste e corresponde de uma equação cúbica. Figura adaptada de $(27)$

expoente crítico. Seja $\lambda \equiv \lim _{\epsilon \rightarrow 0} \frac{\ln f(\epsilon)}{\ln \epsilon}$. Se $\lambda$ existir, este limite será o expoente crítico associado à função $f(\epsilon)$. Então, podemos escrever $f(\epsilon) \propto \epsilon^{\lambda}$.

Voltando ao tema universalidade e olhando para os exemplos utilizados (sistemas fluido e magnético), temos que ambos apresentam comportamento similar perto de uma transição de segunda ordem: a magnetização espontânea $M$ do sistema ferromagnético se aproxima de zero conforme $T \rightarrow T_{c}$ de modo similar que a diferença de densidade entre as fases de vapor e líquido também se torna nula quando $T \rightarrow T_{c}$. Entretanto, o comportamento crítico de uma função termodinâmica não é tão simples e pode ser descrito na forma geral como

$$
f(\epsilon)=A \epsilon^{\lambda}\left(1+B e^{y}+\ldots\right), \quad y<0 .
$$

Dados experimentais mostram que o primeiro termo domina para temperaturas suficientemente próximas ao ponto crítico. Desta forma, um gráfico dilogaritmico permite obter o expoente $\lambda$ por um ajuste linear. A importância dos expoentes críticos reside no fato de que estes podem ser obtidos, enquanto que a função completa não. Além disso, existe um grande número de relações entre os expoentes que levam a fundamentais considerações na Termodinâmica e Mecânica Estatística, 


\begin{tabular}{|c|c|c|c|c|c|}
\hline Expoente & Definição & $\epsilon$ & $P-P_{c}$ & $\rho-\rho_{c}$ & Descrição da quantidade \\
\hline$\alpha^{\prime}$ & $\begin{array}{l}C_{V} \sim(-\epsilon)^{-\alpha^{\prime}} \\
C_{H} \sim(-\epsilon)^{-\alpha^{\prime}}\end{array}$ & $<0$ & 0 & 0 & $\begin{array}{l}\text { calor específico a volume } \\
\text { constante } \\
\text { calor específico com campo } \\
H \text { constante }\end{array}$ \\
\hline$\alpha$ & $\begin{array}{l}C_{V} \sim \epsilon^{-\alpha} \\
C_{H} \sim \epsilon^{-\alpha}\end{array}$ & $\begin{array}{l}>0 \\
>0\end{array}$ & 0 & 0 & $\begin{array}{l}\text { calor específico a volume } \\
\text { constante } \\
\text { calor específico com campo } \\
H \text { constante }\end{array}$ \\
\hline$\beta$ & $\begin{array}{l}\rho_{L}-\rho_{G} \sim(-\epsilon)^{\beta} \\
M \sim(-\epsilon)^{\beta}\end{array}$ & $\begin{array}{l}<0 \\
<0\end{array}$ & 0 & $\begin{array}{l}\neq 0 \\
\neq 0\end{array}$ & $\begin{array}{l}\text { diferença entre as } \\
\text { densidades de líquido e gás } \\
\text { magnetização na ausência } \\
\text { de campo magnético } H\end{array}$ \\
\hline$\gamma^{\prime}$ & $\begin{array}{l}K_{T} \sim(-\epsilon)^{-\gamma^{\prime}} \\
\chi_{T} \sim(-\epsilon)^{-\gamma^{\prime}}\end{array}$ & $\begin{array}{l}<0 \\
<0\end{array}$ & 0 & $\begin{array}{l}\neq 0 \\
\neq 0\end{array}$ & $\begin{array}{l}\text { compressibilidade isotérmica } \\
\text { susceptibilidade isotérmica } \\
\text { na ausência de campo } H\end{array}$ \\
\hline$\gamma$ & $\begin{array}{l}K_{T} \sim \epsilon^{-\gamma} \\
\chi_{T} \sim \epsilon^{-\gamma}\end{array}$ & $\begin{array}{l}>0 \\
>0\end{array}$ & $\begin{array}{l}0 \\
0\end{array}$ & $\begin{array}{l}\neq 0 \\
\neq 0\end{array}$ & $\begin{array}{l}\text { compressibilidade isotérmica } \\
\text { susceptibilidade isotérmica } \\
\text { ausência de campo }\end{array}$ \\
\hline $\begin{array}{l}\delta \\
\delta \\
\end{array}$ & $\begin{array}{l}P-P_{c} \sim\left|\rho_{L}-\rho_{G}\right|^{\delta} \operatorname{sgn}\left(\rho_{L}-\rho_{G}\right) \\
H \sim|M|^{\delta} \operatorname{sgn}(M)\end{array}$ & $\begin{array}{l}0 \\
0 \\
\end{array}$ & $\begin{array}{l}\neq 0 \\
\neq 0\end{array}$ & $\begin{array}{l}\neq 0 \\
\neq 0\end{array}$ & isoterma crítica \\
\hline$\nu^{\prime}$ & $\begin{array}{l}\xi \sim(-\epsilon)^{-\nu^{\prime}} \\
\xi \sim \epsilon^{-\nu}\end{array}$ & $\begin{array}{l}<0 \\
>0\end{array}$ & 0 & $\begin{array}{l}\neq 0 \\
\neq 0\end{array}$ & comprimento de correlação \\
\hline
\end{tabular}

Tabela 2.1: Definições de expoentes críticos para sistemas fluido e magnético (27).

sendo gerais para qualquer sistema. A tabela 2.1 traz um resumo das definições de alguns dos principais expoentes críticos para sistemas fluidos e magnéticos. Os expoentes que levam consigo ' indicam que $T<T_{c}$, ou seja, que a temperatura $T$ tende à temperatura crítica pela esquerda, enquanto que os expoentes que não possuem ' indicam que $T>T_{c}$. O expoente $\beta$, apesar de não ser acompanhado de ', é obtido pelo limite $\epsilon \rightarrow 0^{-}$. 


\subsubsection{Relações entre os expoentes críticos}

Nesta seção veremos que igualdades entre os expoentes críticos podem ser obtidas a partir das relações de escala para potenciais termodinâmicos. A hipótese de escala somente pode ser aplicada às funções homogêneas generalizadas. Portanto, em primeiro lugar é necessário para um bom entendimento, definir o que vem a ser uma função homogênea generalizada e uma transformação de escala para sistemas em equilíbrio. Chamamos de função homogênea de ordem $p$ qualquer função que apresente a seguinte propriedade:

$$
f(\lambda \mathbf{r})=\lambda^{p} f(\mathbf{r}),
$$

com $p$ arbitrário e $\mathbf{r}=(x, y, \ldots)$. A figura 2.4 ilustra uma tranformação de escala de uma função $f(\mathbf{r})$ para uma função $f(\lambda \mathbf{r})$. Entretanto, a hipótese de escala de equilíbrio não assume que os potenciais termodinâmicos apresentem a propriedade de uma função homogênea conforme 2.6, e sim a forma mais geral

$$
f\left(\lambda^{a} x, \lambda^{b} y\right)=\lambda f(x, y),
$$

onde $a$ e $b$ são valores arbitrários. As funções que satisfazem a relação acima são denominadas de funções homogêneas generalizadas.

Agora que definimos o que vem a ser uma tranformação de escala em uma função homogênea generalizada, vamos considerar a energia livre de Gibbs $G(T, H)$ para um sistema magnético. Queremos escrever essa função na forma $G(\epsilon, H)$, onde novamente $\epsilon=\frac{T-T_{c}}{T_{c}}$. A hipótese de escala implica que esta deve ser uma função homogênea generalizada. Utilizando o equação 2.7 temos que devem existir dois parâmetros $a_{\epsilon}$ e $a_{H}$, de forma que

$$
G\left(\lambda^{a_{\epsilon}} \epsilon, \lambda^{a_{H}} H\right)=\lambda G(\epsilon, H),
$$

para qualquer valor de $\lambda$. A partir da equação 2.8 demonstra-se que todos os expoentes críticos podem ser expressos em termos dos dois parâmetros de escala $a_{\epsilon}$ e $a_{H}$. Consequentemente, se se dois expoentes críticos são especificados, é possível obter todos os outros. Diferenciando ambos os lados da equação 2.8 em relação a $H$ e utilizando o fato de que $\partial G / \partial H=M$ temos

$$
\lambda^{a_{H}} M\left(\lambda^{a_{\epsilon}} \epsilon, \lambda^{a_{H}} H\right)=\lambda M(\epsilon, H) .
$$




$$
f(\lambda \mathbf{r})=g(\lambda) \int(\mathbf{r})
$$

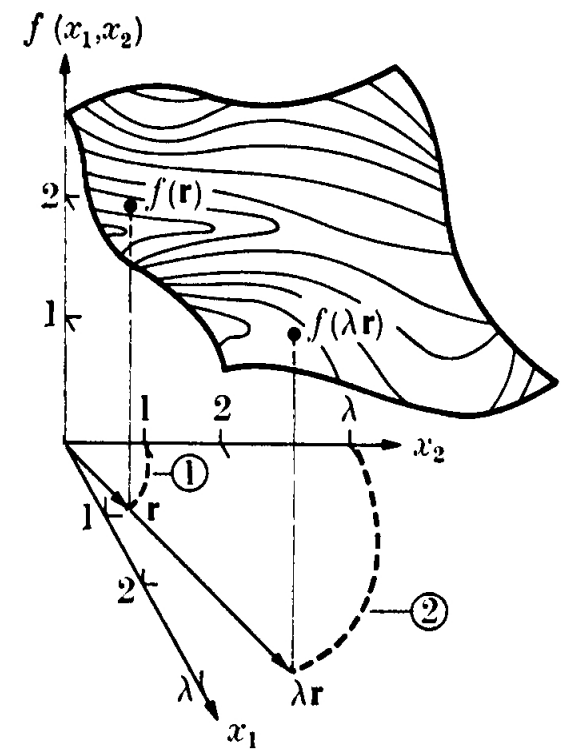

(a)

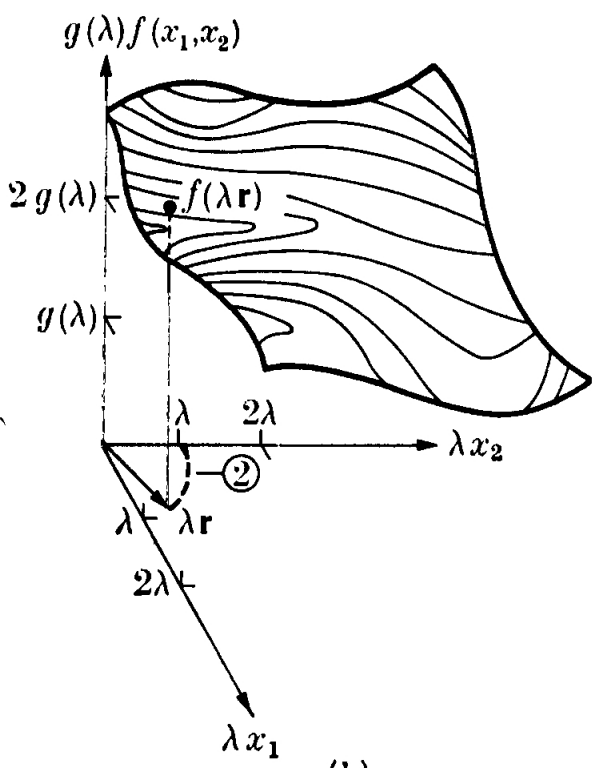

(b)

Figura 2.4: Ilustração de uma mudança de escala de uma função (a) $f(\mathbf{r})$ para uma função (b) $f(\lambda \mathbf{r})$. Figura retirada de (27).

Na ausência de campo magnético, e fazendo $\lambda=(-1 / \epsilon)^{1 / a_{\epsilon}}$, a equação 2.9 se torna

$$
M(\epsilon, 0)=(-\epsilon)^{\left(1-a_{H}\right) / a_{\epsilon}} M(-1,0) .
$$

Da tabela 2.1, sabemos que quando $\epsilon \rightarrow 0^{-}, M(\epsilon, 0) \sim(-\epsilon)^{\beta}$. Desta forma, temos que

$$
\beta=\frac{1-a_{H}}{a_{\epsilon}}
$$

Se, de forma análoga, fizermos $\epsilon=0$ e $H \rightarrow 0$, utilizando $\lambda=\left(-1 / a_{H}\right)$, a equação 2.9 fornece

$$
M(0, H)=H^{\left(1-a_{H}\right) / a_{H}} M(0,1) .
$$

Como, da tabela 2.1, $M(0, H) \sim H^{1 / \delta}$, obtemos

$$
\delta=\frac{a_{H}}{1-a_{H}} .
$$

Resolvendo as equações 2.10 e 2.11 simultaneamente, temos os resultados:

$$
\begin{aligned}
& a_{\epsilon}=\frac{1}{\beta} \frac{1}{\delta+1}, \\
& a_{H}=\delta \frac{1}{\delta+1} .
\end{aligned}
$$




$$
\begin{aligned}
& \alpha+2 \beta+\gamma=2 \\
& \alpha+\beta(\delta+1)=2 \\
& \gamma(\delta+1)=(2-\alpha)(\delta-1) \\
& \gamma=\beta(\delta-1) \\
& \delta=\frac{2-\alpha+\gamma}{2-\alpha-\gamma} \\
& \alpha=\alpha^{\prime} \\
& \gamma=\gamma^{\prime}
\end{aligned}
$$

Tabela 2.2: Algumas igualdades entre expoentes críticos.

Podemos obter também outros expoentes a partir de derivadas de maior ordem de $G$. Como exemplo, derivando duas vezes em relação a $H$ e lembrando que, para $H=0$ e $\epsilon \rightarrow 0^{-}, \chi(\epsilon, 0) \sim(-\epsilon)^{-\gamma^{\prime}}$, obtemos a relação

$$
\gamma^{\prime}=\frac{2 a_{H}-1}{a_{\epsilon}} .
$$

A partir das expressões 2.13 e 2.14 , temos

$$
\gamma^{\prime}=\beta(\delta-1)
$$

Da mesma forma, outras igualdades podem ser obtidas. Na tabela 2.2 temos algumas igualdades preditas pela hipótese de escala.

\subsection{Análise de escala de tamanho finito}

Dado que em uma simulação de Monte Carlo estamos distantes do limite termodinâmico, ou seja, o número de spins é finito e relativamente pequeno, os efeitos de tamanho finito devem ser considerados. Utilizamos a análise de escala de tamanho finito para encontrar valores para os expoentes críticos e para a temperatura crítica a partir da observação do comportamento de quantidades mensuráveis em função do tamanho da rede. Desta forma, podemos variar o tamanho do sistema na simulação e os efeitos de tamanho finito podem ser estimados usando essa teoria.

Ao considerarmos sistemas de tamanho $L$ devemos levar em conta o comportamento do comprimento de correlação $\xi$. Isto faz com que identifiquemos três regiões no espaço dos acoplamentos (14): 
i) uma região onde valores destes acoplamentos estão muito distantes do ponto crítico. Nesta situação podemos afirmar que a rede finita é indistinguível de um sistema associado com o limite termodinâmico, ou seja, $L \gg \xi \approx 1$ (vide figura 2.5a);

ii) pontos na região crítica (ou região de escala) onde a rede finita ainda apresenta o mesmo comportamento de escala que o sistema de volume infinito, ou seja, $L \gg \xi \gg 1$ (vide figura 2.5b). Perto do ponto crítico, o comprimento de correlação é muito grande e somente configurações do sistema da ordem de $\xi$ são importantes;

iii) pontos nos quais $L \approx \xi \gg 1$, e assim observaríamos efeitos significativos (ou efeitos de arredondamento) nas grandezas termodinâmicas de rede finita (vide figura $2.5 \mathrm{c})$.

A teoria de escala de tamanho finito diz que, para uma transição de segunda ordem, a parte singular da energia livre depende somente do tamanho $L$ da rede e do comprimento de correlação $\xi$. Isto conduz às seguintes relações de escala de tamanho finito para a altura do pico do calor específico $C$ e da susceptibilidade $\chi$ :

$$
\begin{aligned}
& C\left(T_{c}(L), L\right) \sim L^{\alpha / \nu} \\
& \chi\left(T_{c}(L), L\right) \sim L^{\gamma / \nu},
\end{aligned}
$$

onde $T_{c}(L)$ são os pontos onde os máximos no calor específico e na susceptibilidade são observados. Temos também que

$$
\begin{aligned}
\Gamma(L) & \sim L^{-1 / \nu} \\
T_{c}(L)-T_{c}(\infty) & \sim L^{-1 / \nu},
\end{aligned}
$$

onde $\Gamma(L)$ é a variância do pico para $C$ ou $\chi$. Veja que, se temos o expoente $\nu$, podemos obter a temperatura crítica para o sistema infinito. Já para uma transição de primeira ordem, o comprimento de correlação $\xi$ se mantém finito. Dessa forma, como $L$ excede $\xi$, as quantidades termodinâmicas próximas a temperatura 


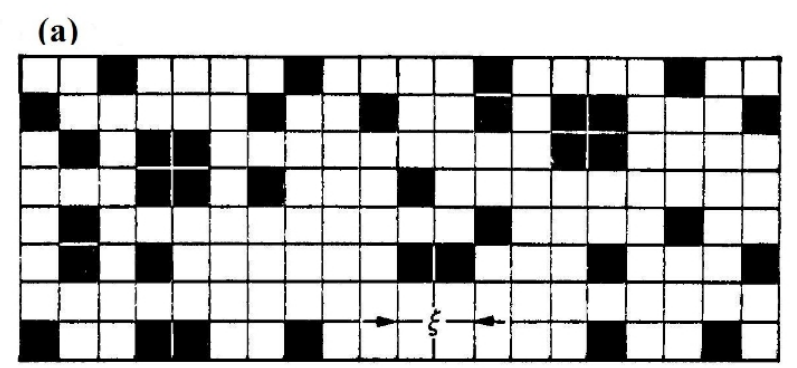

(b)

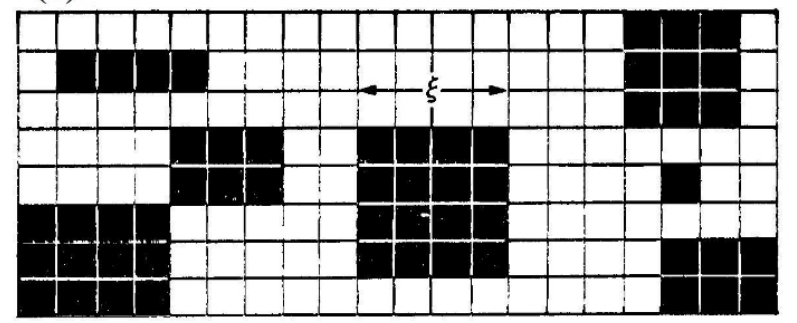

(c)

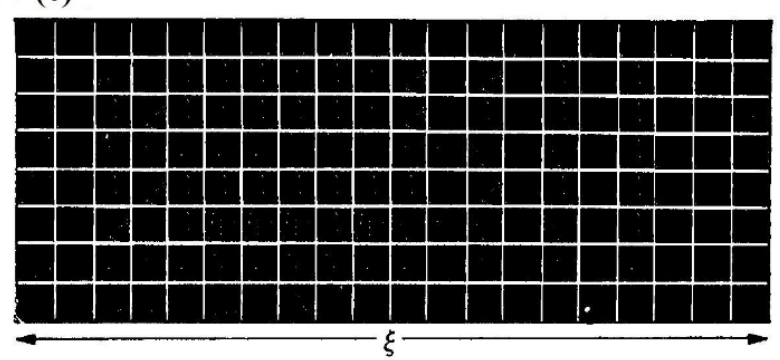

Figura 2.5: Comportamento do comprimento de correlação para temperaturas (a) $T>>T_{c}$, (b) $T \gtrsim T_{c}$, (c) $T=T_{c}$. Figura adaptada de (27).

de transição $T_{c}(L)$ dependem somente do volume do sistema. Então,

$$
\begin{aligned}
C\left(T_{c}(L), L\right) & \sim L^{d} \\
\chi\left(T_{c}(L), L\right) & \sim L^{d} \\
\Gamma(L) & \sim L^{-d} \\
T_{c}(L)-T_{c}(\infty) & \sim L^{-d} .
\end{aligned}
$$

Observe que se $\nu=1 / d$ e $\alpha=\gamma=1$, obtemos as relações para transições de fase de primeira ordem. Isso significa que a análise de escala de tamanho finito pode ser tratada da mesma forma independente da ordem de transição, sendo que a diferenciação da ordem da transição de fase recai sobre os valores dos expoentes críticos. 


\section{Capítulo}

\section{ZEROS DA FUNÇÃO DE PARTIÇÃO}

\subsection{Introdução}

A função de partição canônica $Z$ é uma quantidade importante na obtenção das propriedades estatísticas de um sistema em equilíbrio termodinâmico. Variáveis termodinâmicas do sistema, tais como a energia total, energia livre, entropia e pressão, podem ser expressas em termos da função de partição ou de suas derivadas. A função de partição canônica descreve um sistema que está sujeito a trocar calor com o ambiente à temperatura, volume e número de partículas fixos. Suponhamos ter um sistema deste tipo, vamos rotular com $j(j=1,2,3, \ldots)$ os estados possíveis do sistema e denominar a energia total do microestado $j$ como $E_{j}$. A função de partição canônica é dada por

$$
Z=\sum_{j} e^{-\beta E_{j}}
$$

onde $\beta$ corresponde ao inverso da temperatura, $\beta=\frac{1}{K_{B} T}$, com $K_{B}$ sendo a constante de Boltzmann. A função de partição é dependente das possíveis energias $E_{j}$ do sistema. Por sua vez, cada energia, além de depender de outras grandezas termodinâmicas, tais como o número de partículas e volume, é fortemente dependente dos detalhes microscópicos, como por exemplo, do tipo de interação entre as partículas do sistema. Esta dependência em variáveis microscópicas é o ponto central da Mecânica Estatística, pois a partir de um modelo que forneça informações sobre os componentes microscópicos de um sistema, podemos obter as energias de 
cada microestado e assim obter a função de partição.

Relacionamos a função de partição às propriedades termodinâmicas por meio do seu significado estatístico. A probabilidade $P_{j}$ de que o sistema ocupe o microestado $j$ é

$$
P_{j}=\frac{1}{Z} e^{-\beta E_{j}} .
$$

Note que $Z$ faz o papel de uma constante de normalização da distribuição de probabilidade, ou seja

$$
\sum_{j} P_{j}=\frac{1}{Z} \sum_{j} e^{-\beta E_{j}}=1 .
$$

Dessa forma, o valor esperado para a energia é dado por

$$
\langle E\rangle=\sum_{j} E_{j} P_{j}=\frac{1}{Z} \sum_{j} E_{j} e^{-\beta E_{j}}=-\frac{1}{Z} \frac{\partial}{\partial \beta} Z(\beta)=-\frac{\partial \ln Z}{\partial \beta},
$$

ou, equivalentemente,

$$
\langle E\rangle=K_{B} T^{2} \frac{\partial \ln Z}{\partial T} .
$$

Sendo a entropia

$$
S \equiv-K_{B} \sum_{j} P_{j} \ln P_{j}=K_{B}(\ln Z+\beta\langle E\rangle),
$$

e a energia livre de Helmholtz $F=\langle E\rangle-T S$, obtemos

$$
F=-K_{B} T \ln Z
$$

A variância na energia (ou flutuação de energia) é

$$
(\delta\langle E\rangle)^{2}=\left\langle E^{2}\right\rangle-\langle E\rangle^{2}=\frac{\partial^{2} \ln Z}{\partial \beta^{2}} .
$$

Assim, o calor específico pode ser escrito como

$$
C_{v}=\frac{\partial\langle E\rangle}{\partial T}=\frac{1}{K_{B} T^{2}}(\delta\langle E\rangle)^{2} .
$$

Vimos até agora que a função de partição fornece toda a descrição do sistema e que grandezas como calor específico, entropia e energia livre, podem ser expressas em função desta. Entretanto, a obtenção da função de partição de um sistema não é tão trivial e simples, e para sistemas muito complexos não é factível sua obtenção. Para 
um sistema estatístico de tamanho finito, a função de partição pode ser expressa como um polinômio em um número finito de termos. Uma maneira de visualizar as propriedades desse polinômio é estudar a localização de seus zeros complexos.

Os zeros da função de partição têm sido amplamente utilizados para obter informações sobre transições de fase a partir de simulações de Monte Carlo ou a partir de soluções exatas de sistemas finitos ou mesmo infinitos, mas unidimensionais. Neste contexto, as relações de escala para o primeiro zero permitem a obter o expoente de correlação $\nu$. Também veremos que é possível determinar a ordem da transição de fase olhando para a distribuição dos zeros no plano complexo em sistemas finitos. Para esta finalidade, usaremos neste trabalho a análise de escala de tamanho finito para a obtenção da ordem de transição e da temperatura crítica, com o método proposto em (18) e as aproximações consideradas, por exemplo, em (15) e (19). Nesse método, obtemos o expoente $\nu$ somente a partir da parte imaginária do zero mais próximo do eixo real. Veremos detalhes desse processo mais adiante.

\subsection{Zeros da função de partição}

Vamos iniciar essa seção com o estudo dos zeros complexos da função de partição apresentado no trabalho de Yang e Lee (21), publicado em 1952. Nesse trabalho, os autores mostram que é possível estudar o comportamento analítico de funções termodinâmicas indo ao plano complexo, o qual permite descrever inclusive regiões de transição de fase. A seguir temos uma descrição do trabalho citado, o marco inicial e fundamental que possibilitou esta nova abordagem.

\subsubsection{Zeros de Yang-Lee}

O trabalho de Yang e Lee é dividido em duas partes. A primeira parte (teoria da condensação), inicia-se com o estudo do fenômeno de condensação por meio dos zeros da função de partição no plano da fugacidade. Na segunda parte (gás na rede e modelo de Ising), a equivalência entre o modelo de gás na rede e o modelo de Ising leva ao estudo dos zeros também no plano da fugacidade. As raízes 
complexas da função de partição grande canônica no plano complexo da fugacidade, considerando-se uma classe de condições sobre o potencial de interação entre as partículas, caem sobre um círculo unitário nos dois modelos. Esse resultado ficou conhecido como Teorema do Círculo de Yang e Lee.

Para entender o comportamento analítico de funções termodinâmicas iremos considerar um modelo de gás em uma caixa de volume $V$, mantido a temperatura constante $T$. Se este sistema trocar partículas com um reservatório a um potencial químico $\mu$ por partícula, a probabilidade relativa de se ter $N$ partículas dentro da caixa é dada por

$$
\frac{Z_{N}}{N !} y^{N}
$$

onde

$$
Z_{N}=\int \ldots \int_{V} d \tau_{1} \ldots \tau_{N} \exp \left(-U / K_{B} T\right)
$$

e

$$
y=\left(2 \pi m K_{B} T / H^{2}\right)^{3 / 2} \exp \left(\mu / K_{B} T\right),
$$

onde $h$ é o campo magnético externo.

A função de partição grande canônica $\Xi_{v}$ do gás em um volume $V$ corresponde à soma sobre todos os possíveis números de partículas,

$$
\Xi_{V}=\sum_{N=0}^{M} \frac{Z_{N}}{N !} y^{N},
$$

onde $M$ é o número máximo de partículas que podem ocupar o volume $V$. A pressão e a densidade média do sistema com volume $V$ podem ser calculadas em termos da função de partição grande canônica. Estaremos entretanto interessados em obter equações de estado termodinâmicas e dessa forma tomaremos o limite $V \rightarrow \infty$,

$$
\begin{gathered}
\frac{P}{K_{B} T}=\lim _{V \rightarrow \infty} \frac{1}{V} \ln \Xi_{V}, \\
\rho=\lim _{V \rightarrow \infty} \frac{\partial}{\partial \ln y} \frac{1}{V} \ln \Xi_{V} .
\end{gathered}
$$

Yang e Lee discutem em seu trabalho a existência desses limites. Acreditava-se que na fase gasosa esses limites existiam e que as equações acima forneciam equações 
de estado corretas. Entretanto, os autores provam que tanto 3.12 como 3.13 fornecem uma descrição completa para a equação de estado tanto para o gás quanto para a fase condensada. Para tanto, lançaram mão do fato de que a função de partição pode ser escrita como um polinômio de grau $M$,

$$
\Xi_{V}=\prod_{i=1}^{M}\left(1-\frac{y}{y_{i}}\right),
$$

onde $y_{1}, \ldots, y_{M}$ são as raízes da equação $\Xi_{V}(y)=0$. Como todos os coeficientes do polinômio são positivos, não temos nenhuma raíz real e positiva. A distribuição dessas raízes no limite $V \rightarrow \infty$ fornece o comportamento analítico das funções termodinâmicas no plano complexo $y$.

Se no plano complexo $y$ uma região $R$ contendo um segmento do eixo real positivo é sempre livre de raízes, então, nessa região, no limite $V \rightarrow \infty$ todas as quantidades $\frac{1}{V} \ln \Xi_{V}, \frac{\partial}{\partial \ln y} \frac{1}{V} \ln \Xi_{V}, \frac{\partial^{2}}{\partial \ln y^{2}} \frac{1}{V} \ln \Xi_{V}, \ldots$, são analíticas em relação a $y$. Como consequência temos a seguinte equação de estado

$$
\rho=\frac{\partial}{\partial \ln y}\left(P / K_{B} T\right) \text {. }
$$

Porém, nem sempre o limite $\lim _{V \rightarrow \infty}\left(\frac{\partial}{\partial \ln y}\right)$ existe (isto é claro na condensação, onde a densidade não possui um valor único). Temos dessa forma, dois casos:

i) As raízes não se aproximam ou, caso se aproximem, não cruzam o eixo real positivo no limite de $V \rightarrow \infty$, ou seja, há uma região $R$ que contém todo o eixo positivo e livre de raízes. Nesse caso a pressão e a densidade são funções analíticas de $y$, ou seja, o sistema é composto de uma única fase (veja a figura 3.1a.)

ii) As raízes cruzam o eixo real positivo no limite $V \rightarrow \infty$. Se considerarmos que isso ocorre nos pontos $y=t_{1}, t_{2}$, teremos 3 regiões $R_{1}, R_{2}$ e $R_{3}$ livre de raízes (veja a figura $3.1 \mathrm{~b}$ ). Nos pontos $t_{1}$ e $t_{2}$, teremos em geral uma descontinuidade na densidade $\rho$.

Conforme variamos a temperatura do sistema, os pontos $t_{1}$ e $t_{2}$ se movem ao longo do eixo real. Se, a uma certa temperatura, as raízes cruzam o eixo real em um dos 


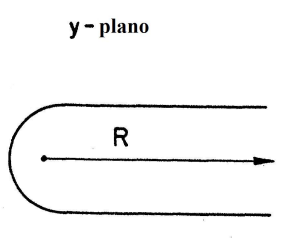

(a)

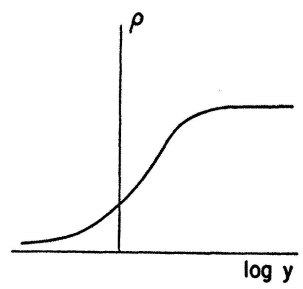

(c)

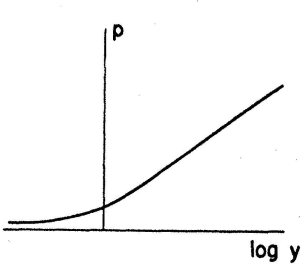

(b)

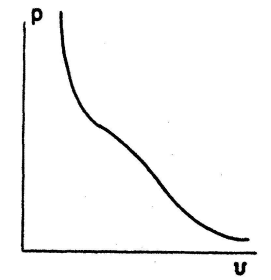

(d)

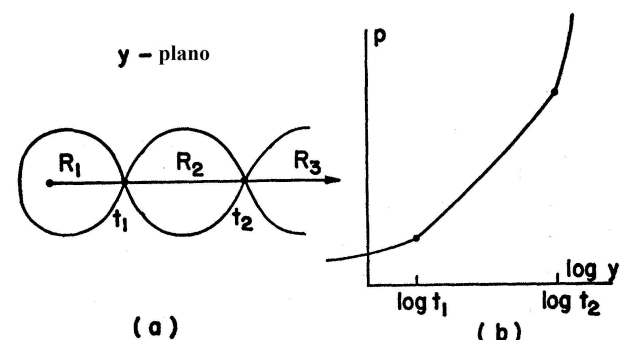

(a)

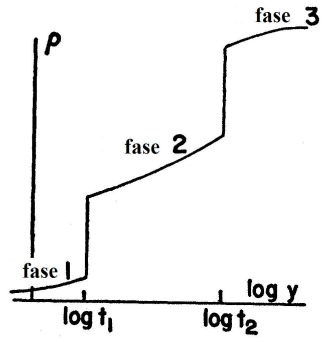

(c)

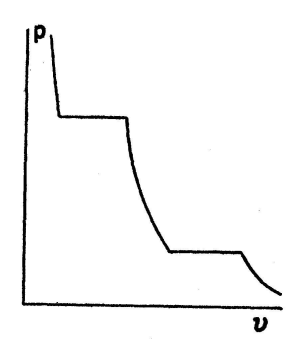

(d)

(a)

(b)

Figura 3.1: Comportamento analítico a uma dada temperatura das funções termodinâmicas para: (a) um sistema em uma única fase. A região $R$ nesse caso é livre de raízes; (b) um sistema que passa por duas transições de fase em $t_{1}$ e $t_{2}$, pontos em que ocorrem as raízes da função de partição no limite termodinâmico. A região $R_{1}, R_{2}$ e $R_{3}$ são livres de raízes e correspondem às diferentes fases do sistema. Figuras adaptadas de (21).

dois pontos, por exemplo, o ponto $t_{1}$, então essa é a temperatura crítica $T_{c}$ para a transição da fase 1 para a 2. Se, entretanto, as raízes cruzarem o eixo real em um ponto $t_{0}$ diferente de $t_{1}$ e $t_{2}$, teremos um ponto triplo para essa temperatura.

Vimos que os zeros da função de partição nem sempre são indicativos de singularidades em quantidades termodinâmicas. Elas podem ser interpretadas como linhas que separam diferentes comportamentos assintóticos da função de partição no limite termodinâmico. Em geral, ao longo dessas linhas a parte real da continuação analítica e da energia livre será contínua enquanto que a descontinuidade da parte imaginária é proporcional à densidade dos zeros (14). Como veremos na próxima seção, o algoritmo multicanônico estima a densidade de estados $\rho(E)$ do sistema, a partir da qual podemos construir a função de partição. Assumindo um espectro de energia discreto, temos

$$
Z(\beta)=\sum_{E} \rho(E) u^{E}
$$


onde $u=e^{-\beta}$. Neste caso, estaremos estudando os zeros em outra variável: a temperatura. A obtenção desses zeros nos permite determinar o comportamento crítico do modelo e estimar assim, a partir de expoentes críticos, a ordem da transição de fase e a temperatura crítica. Veremos a seguir um estudo mais detalhado desses zeros.

\subsubsection{Zeros de Fisher}

Os zeros complexos da função de partição na ausência de campo magnético externo, ou para campo fixo, podem ser obtidos pela extensão complexa da variável temperatura. Não há nenhum resultado simples para antever a localização destes zeros, ou seja, não há nenhum teorema equivalente ao Teorema do Círculo para os zeros de Fisher. Entretanto, suas localizações e comportamentos no plano complexo da temperatura determinam as características críticas do sistema. Diferentes transições de fase podem ser classificadas de acordo com o comportamento da densidade limite de zeros complexos na variável temperatura. Da definição da função de partição em termos dos zeros complexos,

$$
Z(\beta)=\prod_{i=1}^{M}\left(1-\frac{\beta}{\beta_{i}}\right),
$$

e aplicando $\ln$ em ambos os lados

$$
\ln Z(\beta)=\sum_{i=1}^{M} \ln \left(1-\frac{\beta}{\beta_{i}}\right),
$$

obtemos a energia do sistema,

$$
E(\beta)=\sum_{i=1}^{M} \frac{1}{\beta_{i}-\beta} .
$$

Considerando a definição do calor específico 3.7 obtemos

$$
C_{v}=\beta^{2} \sum_{i=1}^{M} \frac{1}{\left(\beta_{i}-\beta\right)^{2}} .
$$

Tais grandezas não exibem singularidades reais, a menos que $V \rightarrow \infty$, ou seja, $M \rightarrow \infty$. Dessa forma, podemos dizer que sistemas finitos não exibem transições de 
fase. Entretanto, a distribuição dos zeros complexos da função de partição pode ser usada para classificar transições de fase em sistemas finitos (28). A energia livre 3.6 é analítica em qualquer região do plano complexo da temperatura, exceto nos zeros de $Z(\beta)$. Como já vimos para o caso dos zeros no plano da fugacidade, se os zeros formam uma linha densa no plano complexo da temperatura, essas linhas separam diferentes fases do sistema. Então, a distribuição de zeros contém toda a informação sobre o sistema e as funções termodinâmicas são derivadas desta distribuição. Os zeros da função de partição são polos de $F$ e do calor específico 3.20. A maior contribuição para singularidades do calor específico vem dos zeros próximos ao eixo real, ou seja, zeros que encostam no eixo real no limite de $V \rightarrow \infty$ causam uma divergência no calor específico. Para caracterizar a distribuição dos zeros próximos ao eixo real, vamos considerar que, nessa região, os zeros complexos na variável temperatura estão sobre uma linha reta e que o ângulo dessa reta com o eixo imaginário (vide figura 3.2) é dado por $\theta=\arctan (\gamma)$, sendo $\gamma$ dado po (28)

$$
\gamma=\frac{\operatorname{Re}\left(\beta_{2}\right)-\operatorname{Re}\left(\beta_{1}\right)}{\operatorname{Im}\left(\beta_{2}\right)-\operatorname{Im}\left(\beta_{1}\right)}
$$

Dessa forma, é possível obter o ponto onde os zeros cruzam o eixo real no limite termodinâmico ,

$$
\beta_{c u t}=\operatorname{Re}\left(\beta_{1}\right)-\gamma \operatorname{Im}\left(\beta_{1}\right) .
$$

Se definirmos a densidade discreta da distribuição dos zeros $\phi$ em torno do $k$-ésimo zero como sendo a média do inverso da distância entre este zero e seus dois vizinhos mais próximos, ou seja

$$
\phi\left(\operatorname{Im}\left(\beta_{k}\right)\right)=\frac{1}{2}\left(\frac{1}{\left|\beta_{k}-\beta_{k-1}\right|}+\frac{1}{\left|\beta_{k+1}-\beta_{k}\right|}\right),
$$

com $k=2,3,4 \ldots$, podemos obter uma aproximação para o expoente $\alpha$. Devido ao fato de que a importância dos zeros para o calor específico diminui com o aumento de $\operatorname{Im}(\beta)$, então, para uma região de $\operatorname{Im}(\beta)$ pequeno podemos propor: $\phi \propto \operatorname{Im}(\beta)^{\alpha}$, onde $\alpha$ é o expoente crítico para o calor específico. Considerando os primeiros zeros temos:

$$
\alpha=\frac{\ln \phi_{3}-\ln \phi_{2}}{\ln \left[\operatorname{Im}\left(\beta_{3}\right)\right]-\ln \left[\operatorname{Im}\left(\beta_{2}\right)\right]} .
$$




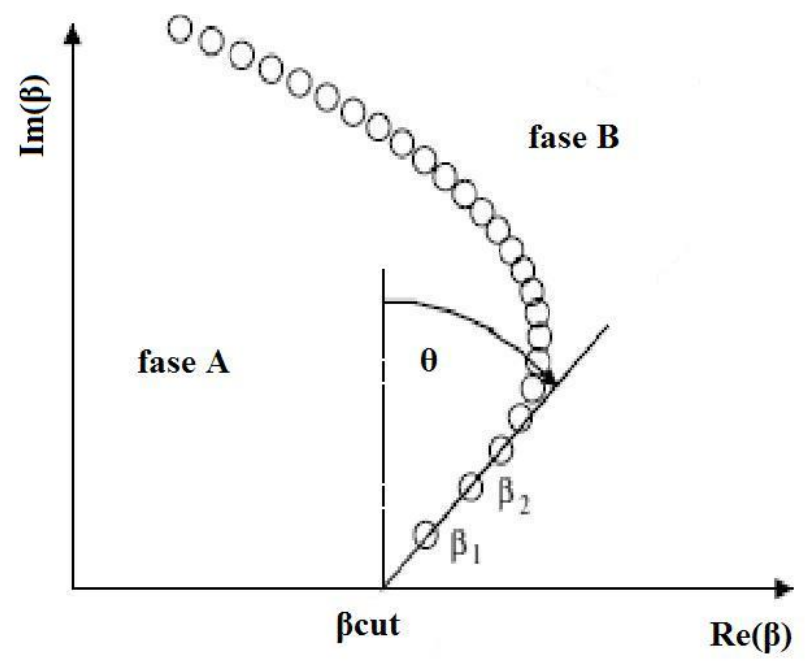

Figura 3.2: Ilustração da distribuição de zeros da função de partição próximos ao eixo real positivo. Os parâmetros utilizados na classificação da ordem de transição são indicados $\left(\beta_{\text {cut }}, \beta_{1}, \beta_{2}\right.$ e o ângulo $\left.\nu\right)$. Figura adaptada de (28).

Note que, na verdade, para utilizarmos tal relação, necessitamos ter em mãos os quatro primeiros zeros, pois

$$
\phi_{2}=\frac{1}{2}\left(\frac{1}{\left|\beta_{2}-\beta_{1}\right|}+\frac{1}{\left|\beta_{3}-\beta_{2}\right|}\right),
$$

$\mathrm{e}$

$$
\phi_{3}=\frac{1}{2}\left(\frac{1}{\left|\beta_{3}-\beta_{2}\right|}+\frac{1}{\left|\beta_{4}-\beta_{3}\right|}\right) .
$$

A partir da parte imaginária $\operatorname{Im}(\beta)$ dos zeros mais próximos ao eixo real e dos parâmetros $\alpha$ e $\gamma$ podemos classificar a ordem da transição considerando o fato de que, para uma transição de fase verdadeira, $\operatorname{Im}\left(\beta_{1}\right) \rightarrow 0$. Dessa forma a transição de fase pode ser classificada a partir de $\alpha$ e $\gamma$ como segue (28):

- se $\alpha=0$ e $\gamma=0$ : primeira ordem,

- se $0<\alpha<1$ e $\gamma$ arbitrário: segunda ordem,

- se $\alpha>1$ e $\gamma$ arbitrário: ordem maior. 


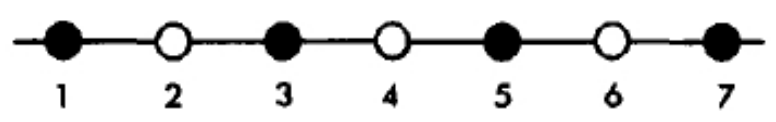

Figura 3.3: Ilustração de um pequeno pedaço da rede de spins para o Modelo de Ising 1D. As somas sobre as variáveis pares de spin (círculos brancos) são efetuadas primeiro. Este procedimento fornece uma nova soma agora em função das variáveis ímpares de spin (círculos pretos).

\subsection{Escala de tamanho finito para os zeros da função de partição}

Vimos que grandezas termodinâmicas apresentam relações de escala com o tamanho do sistema e que podem ser descritas em termos dos zeros complexos da função de partição (18). Consequentemente também existem relações de escala de tamanho finito para os zeros responsáveis pelo comportamento singular destas funções termodinâmicas. Antes de iniciarmos a demonstração das relações de escala de tamanho finito para os zeros, é necessário primeiro entender o que vem a ser o grupo de renormalização.

\subsubsection{Grupo de renormalização}

Apresentaremos esse conceito utilizando um modelo bem mais simples que o nosso: o modelo de Ising unidimensional, como proposto em (29). Se considerarmos a figura 3.3 temos que a função de partição é dada por:

$$
Z=\sum \exp \left[K\left(\sigma_{1} \sigma_{2}+\sigma_{2} \sigma_{3}+\sigma_{3} \sigma_{4}+\sigma_{4} \sigma_{5}+\ldots\right)\right],
$$

onde a soma é feita sobre todos as possíveis configurações do sistema, ou seja sobre todos os possíveis valores para $\sigma_{1}, \sigma_{2}, \ldots, \sigma_{i}$, etc, com $\sigma_{i}= \pm 1$ e $K=J / K_{B} T$. Se escrevermos a função de partição como

$$
Z=\sum e^{K\left(\sigma_{1} \sigma_{2}+\sigma_{2} \sigma_{3}\right)} e^{K\left(\sigma_{3} \sigma_{4}+\sigma_{4} \sigma_{5}\right) \ldots},
$$

é fácil perceber que o termo $\sigma_{2}$ aparece somente na primeira exponencial. Assim, se fizermos a soma em $\sigma_{2}$ teremos

$$
Z=\sum\left[e^{K\left(\sigma_{1}+\sigma_{3}\right)}+e^{-K\left(\sigma_{1}+\sigma_{3}\right)}\right] e^{K\left(\sigma_{3} \sigma_{4}+\sigma_{4} \sigma_{5}\right) \cdots} .
$$


Se continuarmos somando sobre todos $\sigma_{i}$, com $i$ par, obtemos

$$
Z=\sum\left[e^{K\left(\sigma_{1}+\sigma_{3}\right)}+e^{-K\left(\sigma_{1}+\sigma_{3}\right)}\right]\left[e^{K\left(\sigma_{3}+\sigma_{5}\right)}+e^{-K\left(\sigma_{3}+\sigma_{5}\right)}\right] \ldots
$$

A soma agora ficou restrita às variáveis de spin $\sigma_{j}$, com $j$ ímpar. A ideia do grupo de renormalização é obter a função de partição com um acoplamento $K$ em termos de uma nova função de partição tendo acoplamento $K^{\prime}$. Então, vamos procurar um valor de $K^{\prime}$ e uma função $f$ que satisfaça:

$$
e^{K\left(\sigma_{1}+\sigma_{3}\right)}+e^{-K\left(\sigma_{1}+\sigma_{3}\right)}=f(K) e^{K^{\prime} \sigma_{1} \sigma_{3}}
$$

para todos os valores possíveis de $\sigma_{1}$ e $\sigma_{3}$. A solução para o modelo de Ising em uma dimensão é:

$$
\begin{gathered}
K^{\prime}=(1 / 2) \ln [\cosh (2 K)] \\
f(K)=2 \cosh ^{1 / 2}(2 K) .
\end{gathered}
$$

Agora podemos escrever a função de partição em função de $f(K)$ e $K^{\prime}$

$$
Z=\sum f(K) e^{K^{\prime} \sigma_{1} \sigma_{3}} f(K) e^{K^{\prime} \sigma_{3} \sigma_{5}} \ldots=f(K)^{N / 2} e^{K^{\prime}\left(\sigma_{1} \sigma_{3}+\sigma_{3} \sigma_{5}+\ldots\right)},
$$

onde $N$ é o número de partículas do sistema. A partir desse resultado mostramos que a função de partição $Z(N, K)$ para um sistema com $N$ spins interagindo por um acoplamento $K$ pode ser relacionada com a função de partição $Z\left(N / 2, K^{\prime}\right)$ para um sistema com $N / 2$ spins e constante de acoplamento $K^{\prime}$,

$$
Z(N, K)=f(K)^{N / 2} Z\left(N / 2, K^{\prime}\right)
$$

Da mesma forma, como a energia livre $F$ é proporcional ao tamanho do sistema, temos que

$$
\ln Z=N \zeta(K)
$$

onde $\zeta$ é função do acoplamento $K$. Agora que já temos uma idéia do que vem a ser uma transformação por grupo de renormalização podemos prosseguir com a obtenção das relações de escala de tamanho finito para os zeros da função de partição. 


\subsubsection{Escala de tamanho finito}

A dedução a seguir utiliza o fato de já conhecermos o comportamento dos acoplamentos do modelo sob transformações de escala de tamanho $L$. Os acoplamentos $K_{1}$ e $K_{2}$ são, neste caso, relacionados com a variável temperatura e campo magnético, respectivamente. Podemos expressar as propriedades de transformação do grupo de renormalização da função de partição sob uma mudança no comprimento de escala como:

$$
Z_{L}\left(K_{1}, \ldots\right)=Z_{\frac{L}{b}}\left(K_{1} b^{y_{1}}, \ldots\right) c\left(K_{1}, K_{2}, \ldots\right)
$$

onde $K_{1}, K_{2}, \ldots$ são os acoplamentos reduzidos em torno do ponto crítico e $b$ corresponde a uma transformação de escala: $L \rightarrow \frac{L}{b}$. Note que, como o termo $c$ nunca é nulo, os zeros de $Z_{L}$ são os mesmos que os zeros de $Z_{\frac{L}{b}}$. Se definirmos $\lambda$ como uma fração fixa de $L$, ou seja. $\frac{L}{b}=\frac{1}{\lambda}$, obtemos

$$
Z_{\frac{1}{\lambda}}\left(K_{1} L^{y_{1}} \lambda^{y_{1}}, \ldots\right)=Q\left(x_{1}, x_{2}, \ldots\right)
$$

onde $x_{i}=K_{i} L^{y_{i}}$ são novas variáveis de escala e a função de escala $Q$ é uma função analítica de seus argumentos quando estes são muito pequenos (o que é verdade na região de escala). Como já mencionado, se conhecermos os dois campos de escala principais, temperatura e campo magnético $H$, podemos identificar os expoentes como:

$$
\begin{gathered}
y_{t}=\frac{1}{\nu}, \\
y_{h}=\frac{\beta \delta}{\nu}=\frac{(\beta+\gamma)}{\nu} .
\end{gathered}
$$

Assim, desprezando o efeito de outros operadores, nessa aproximação temos um resultado de escala muito simples para os zeros da função de partição,

$$
Q\left(x_{t}, x_{h}\right)=Q\left(K L^{\frac{1}{\nu}}, H L^{\frac{\beta \delta}{\nu}}\right)=0
$$

onde $K=u-u_{c}, u=\exp (-\kappa \beta)$, e $\kappa$ é uma constante dependente do modelo e escolhida de forma a reescalonar a energia para tornar a função de partição um polinômio na variável $u$. Para os modelos de Ising bi e tridimensional, é conveniente 
usar $\kappa=4$. Para o modelo de Ising bidimensional com interação dipolar usamos $\kappa=1$. Dessa forma, se especificarmos nossa hamiltoniana para um modelo de spins com campo magnético externo, a função de partição pode ser escrita como um polinômio da forma

$$
Z(u, y)=\sum_{M} \sum_{E} n(E, M) u^{E} y^{M}
$$

$\operatorname{com} y=e^{-2 \beta H}$.

Como a função de partição é par em $H, Q$ só pode ser função de $H^{2}$. Com a hipótese de que $H^{2}$ é suave, é possível resolver a equação 3.41 e ter como resultado:

$$
H^{2} L^{\frac{2 \beta \delta}{\nu}}=f_{i}\left(K L^{\frac{1}{\nu}}\right)
$$

onde $i$ rotula a $i$-ésima raíz. Se por hipótese $f_{i}(x)$ é uma função analíticas em $x$ podemos inverter a relação acima e obter

$$
K_{i} L^{\frac{1}{\nu}}=f_{i}^{-1}\left(H^{2} L^{\frac{2 \beta \delta}{\nu}}\right)
$$

e no caso de $h=0$,

$$
K_{i}=L^{\frac{-1}{\nu}} f_{i}^{-1}(0)
$$

Temos então que, para $L$ suficientemente grande, o zero mais próximo do eixo real $u$ no plano complexo obedece a seguinte relação (15):

$$
u_{1}^{0}(L)=u_{c}+A L^{-1 / \nu}\left[1+O(L)^{-\omega}\right]
$$

$\operatorname{com} \omega>0$. Uma forma de se obter o valor do expoente $\nu$ é fazer uma regressão linear dos resultados de $u_{1}^{0}(L)$ na forma

$$
\left|u_{1}^{0}(L)-u_{c}\right|=a_{1} L^{-1 / \nu(L)}
$$

para sucessivos valores de $L$ e depois usar esses valores para obter $\nu$ pela seguinte expressão

$$
\nu(L)=\nu+a_{4} L^{-a_{5}} .
$$

Entretanto, para $L$ suficientemente grande podemos utilizar a seguinte regressão linear (30):

$$
-\ln \left|u_{1}^{0}(L)-u_{c}\right|=\frac{1}{\nu} \ln (L)+a .
$$


É óbvio que para muitos modelos, inclusive o modelo do presente trabalho, não conhecemos o valor de $u_{c}$. Dessa forma, uma aproximação válida é substituir $\left|u_{1}^{0}-u_{c}\right|$ pela parte imaginária do primeiro zero $\operatorname{Im}\left(u_{1}^{0}\right)$. Essa aproximação considera que para o primeiro zero, o valor da parte real de $u$ está bem próximo do valor de $u_{c}$. A temperatura crítica pode ser obtida a partir da relação

$$
\operatorname{Re}\left[\beta_{1}^{0}(L)\right]=\beta_{c}+b L^{\frac{-1}{\nu}}
$$

Como vimos no capítulo 3 , se $d \nu=1$, temos uma transição de primeira ordem. Dessa forma, a análise de escala de tamanho finito para os zeros, além de permitir a obtenção da temperatura crítica, também fornece a ordem da transição de fase. 


\section{CAPÍTUlO}

\section{O MODELO DE ISING DIPOLAR BIDIMENSIONAL}

\subsection{Introdução}

Apresentaremos neste capítulo o modelo de Ising dipolar bidimensional utilizado neste trabalho. Conforme discutimos no capítulo 1, este modelo descreve a formação de domínios magnéticos em filmes ultrafinos. A hamiltoniana do modelo apresentada no capítulo 1 contém, além do termo de troca entre os primeiros vizinhos, um termo de interação entre os dipolos magnéticos dos spins. Este termo extra na hamiltoniana fornece um diagrama de fase muito rico, o qual pode ser antecipado por cálculos teóricos. Veremos que existem algumas divergências entre resultados obtidos em diferentes trabalhos envolvendo simulações de Monte Carlo. Dessa forma, além de descrevermos o modelo, também apresentaremos os resultados para o diagrama de fase já existente na literatura. Daremos um enfoque especial à região do diagrama de fase que se estende de $\delta=0.85$ até $\delta=1.3$, pois esta região deve conter um ponto tricrítico cuja determinação é o objetivo deste trabalho.

\subsection{A hamiltoniana do modelo}

Os momentos magnéticos locais de filmes finos podem ser tratados aproximadamente como variáveis de Ising. Uma descrição realística de um filme fino magnético deve incluir interações dipolares de longo alcance. No modelo 
consideramos uma rede formada por uma camada de spins distribuídos regularmente (uma aproximação para monolayers). Os spins possuem configurações limitadas à direção perpendicular ao plano do filme. No modelo de Ising, consideramos somente a interação de troca entre primeiros vizinhos. No modelo de Ising com interação dipolar, um novo termo é adicionado à hamiltoniana do sistema para incluir as interações entre os momentos de dipolo magnético dos spins. A distância entre cada sítio vizinho é considerada igual a 1. A hamiltoniana é dada então pela soma dos dois termos,

$$
H=H_{\text {Ising }}+H_{\text {dipolar }}
$$

onde

$$
\begin{gathered}
H_{\text {Ising }}=-J \sum_{<i, j>} s_{i} s_{j}, \\
H_{\text {dipolar }}=\frac{g}{2} \sum_{i \neq j} \frac{\mu_{i} \mu_{j}}{r_{i j}^{3}} .
\end{gathered}
$$

A variável $s_{i}$ representa o $i$-ésimo spin, $J$ é a constante de interação do Ising puro, $\mu_{i}$ é o momento de dipolo magnético do $i$-ésimo spin e $g$ é a constante de interação entre os momentos de dipolo magnéticos. Os momentos de dipolo magnéticos dos pares de spins $i$ e $j$ localizam-se em $\overrightarrow{r_{i}}$ e $\overrightarrow{r_{j}}$, com $r_{i j}=\left|\overrightarrow{r_{i}}-\overrightarrow{r_{j}}\right|$. Note que o termo dipolar tem o fator $1 / 2$ para que a soma contabilize cada par uma única vez. Se considerarmos a segunda soma efetuada somente entre pares distintos, teremos $H_{\text {dipolar }}=g \sum_{i<j} \frac{\mu_{i} \mu_{j}}{r_{i j}^{3}}$. Podemos ainda escrever o spin como $s_{i}=\sigma_{i} s_{\text {ef }}$ e o momento magnético como $\mu_{i}=$ $\sigma_{i} \mu_{e f}$, sendo o estado do spin especificado pela variável $\sigma_{i}= \pm 1$. As quantidades $s_{e f}$ e $\mu_{e f}$ correspondem ao spin efetivo e ao momento magnético efetivo, respectivamente. Assim, a hamiltoniana passa a ser escrita da seguinte forma:

$$
H=-J s_{e f}^{2} \sum_{<i, j>} \sigma_{i} \sigma_{j}+g \mu_{e f}^{2} \sum_{i<j} \frac{\sigma_{j} \sigma_{j}}{r_{i j}^{3}} .
$$

Dividindo todos os termos da hamiltoniana por $\mu_{e f}^{2}$, teremos a energia na forma adimensional,

$$
E(\sigma)=-\delta \sum_{<i, j>} \sigma_{i} \sigma_{j}+\sum_{i<j} \frac{\sigma_{i} \sigma_{j}}{r_{i j}^{3}}
$$


onde $\delta=\frac{J}{g}\left(\frac{s_{e} f}{\mu_{e} f}\right)^{2}$ é a razão entre os acoplamentos das interações de troca e dipolar. Dessa forma, caracterizamos o sistema em termos de um único parâmetro $(\delta)$ e podemos construir um diagrama de fase em função de um único parâmetro.

\subsection{Diagrama de fase}

Cálculos baseados em aproximações contínuas e sobre redes quadradas levam à conclusão de que o estado fundamental consiste de faixas alternadas de spins caracterizadas por ter todos os spins orientados para baixo ou para cima desde que a interação de troca exceda um valor positivo e pequeno, para $J>0$. Do contrário, o estado ligado é o estado ferromagnético. Esse pequeno valor, acima do qual ocorre a formação da fase do tipo faixas é $\delta=0.425$, conforme cálculos analíticos na ref. (4), enquanto que temos $\delta=0.440313$ na ref. (31). Podemos explicar esse fenômeno de formação de faixas magnéticas conforme segue. As interações ferromagnéticas favorecem o alinhamento dos spins em uma mesma direção. Por outro lado, as interações dipolares (antiferromagnéticas) favorecem o alinhamento antiparalelo entre os spins. A existência de competição entre esses dois tipos de interação causa o fenômeno chamado de "frustração", fornecendo um cenário fenomenologicamente rico. A orientação anisotrópica favorece apenas duas orientações com direções mutuamente perpendiculares para baixas temperaturas. Os resultados analíticos apresentados em (12) sobre a dependência da largura das faixas $h$ com $\delta$ é mostrado na tabela 4.2. A largura $h$ aumenta com a intensidade da interação de troca e é sempre estável em relação à fase ferromagnética, independente de quão grande seja o parâmetro de troca (32).

A partir de simulações de Monte Carlo para $\delta=3$ e 4.45 , Booth e coautores (32) observaram a existência de três fases distintas em função da temperatura para o modelo: a fase do tipo faixas para baixas temperaturas, uma fase intermediária com domínios magnéticos irregulares, mas bem definidos, que denominaram líquido tetragonal, e a fase desordenada paramagnética para altas temperaturas. A transição entre a fase tetragonal e a fase desordenada foi caracterizada como contínua. No mesmo ano, Abanov e coautores (33), por meio de um estudo analítico de limite 


\begin{tabular}{|c|c|}
\hline$h$ & $\delta$ \\
\hline \hline 1 & $0.440313-1.258539$ \\
2 & $1.258539-2.172456$ \\
3 & $2.172456-2.814495$ \\
4 & $2.814495-3.304242$ \\
5 & $3.304242-3.699024$ \\
6 & $3.699024-4.029349$ \\
7 & $4.029349-4.313172$ \\
8 & $4.313172-4.561907$ \\
9 & $4.561907-4.783242$ \\
10 & $4.783242-4.982600$ \\
\hline
\end{tabular}

Tabela 4.1: Dependência entre a largura das faixas e o acoplamento $\delta$.

contínuo para a hamiltoniana, analisaram a dependência na temperatura. Foram observadas as seguintes fases: um cristal esmético com domínio de estruturas do tipo faixas, uma fase intermediária denominada de Ising nemática e o líquido tetragonal (observado também em (32) em simulações de Monte Carlo). As predições teóricas indicaram que a largura das faixas do cristal esmético é altamente dependente da temperatura, a faixa pode adquirir o tamanho de toda a amostra a baixas temperaturas, o que foi verificado também experimentalmente. Para temperaturas maiores que uma temperatura crítica, o sistema passa para uma configuração planar paramagnética. Podemos sintetizar os resultados apresentados em (33), com relação ao tipo de transição de fase em função do aumento da temperatura em duas possibilidades:

i) o cristal esmético passa para a fase nemática através de uma transição do tipo Kosterlitz-Thouless (KT). A fase nemática, por sua vez, passa para a fase de líquido tetragonal por uma transição contínua ou

ii) o cristal esmético passa diretamente para a fase de líquido tetragonal por uma transição de primeira ordem.

A figura 4.1 ilustra as configurações de spin obtidos em diferentes temperaturas 


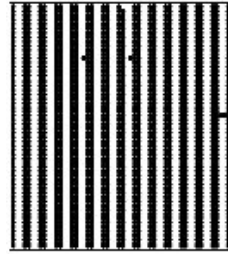

(a)

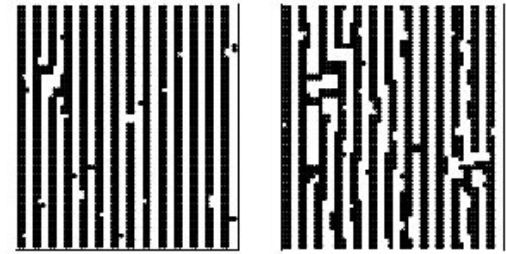

(b) (c)

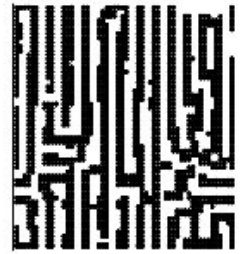

(d)

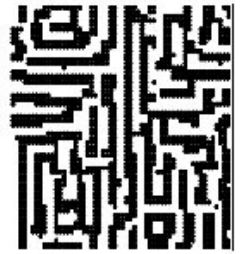

(e)

Figura 4.1: Configurações de spin nas temperaturas de transição para uma rede $L=$ 56 para $\delta=2$. (a) fase do tipo faixas, (b) sistema na temperatura de transição da fase de faixas para a fase nemática, (c) fase nemática, (d) sistema na temperatura de transição da fase nemática para o líquido tetragonal, (e) líquido tetragonal. Figura retirada de (13).

e dando origem às diferentes fases mencionadas acima. Os resultados foram obtidos por simulações de Monte Carlo para a rede de tamanho $L=56$ e $\delta=2$, conforme a ref. (13). Em (34), as fases nemática e tetragonal são definidas a partir dos defeitos topológicos denominados desclinações e deslocações (vide figura 4.2). A desvinculação de pares de deslocações ligados dá origem à fase nemática. Já a origem do líquido tetragonal vem da desvinculação de desclinações. $\quad \operatorname{Em}(9,10)$ Cannas e coautores, utilizando simulações de Monte Carlo, obtiveram resultados condizentes com os já previstos em (33): antes de alcançar o estado paramagnético à altas temperaturas, o sistema passa da fase esmética para a fase tetragonal por uma transição de fase de primeira ordem. Nessa transição da fase esmética para a tetragonal, o sistema pode ainda passar por uma fase intermediária: a fase nemática. A conclusão obtida é que existem os dois cenários já previstos por Abanov, entretanto, com comportamento crítico muito mais complexo, dependente de $\delta$. A transição da fase do tipo faixas para a fase nemática apresenta características incomuns, algumas indicando ser de primeira ordem, outras indicando ser do tipo KT. O diagrama de fase mostrado na fig. 4.3, adaptado de (11), foi construído com base em simulações de Monte Carlo para acoplamentos $\delta$ variando de 1 a 3 , com um foco especial em $\delta=2(h=2)$, e em resultados já existentes na literatura. A fase nemática parece ocorrer em regiões próximas às linhas de transição dentre diferentes fases do tipo faixas, com exceção da transição de $h=1$ para $h=2$. Há nesse diagrama a presença de um ponto tricrítico, cuja literatura indica até o presente momento estar localizado no intervalo $\delta \in[0.85,1]$. Na ref. (10), através 
(a)

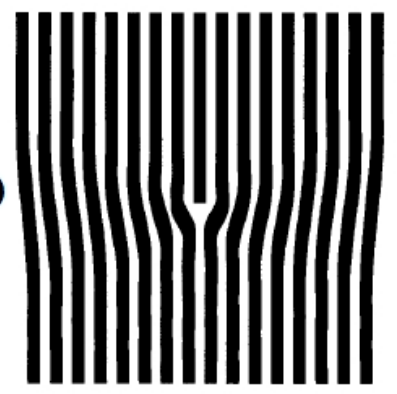

(b)
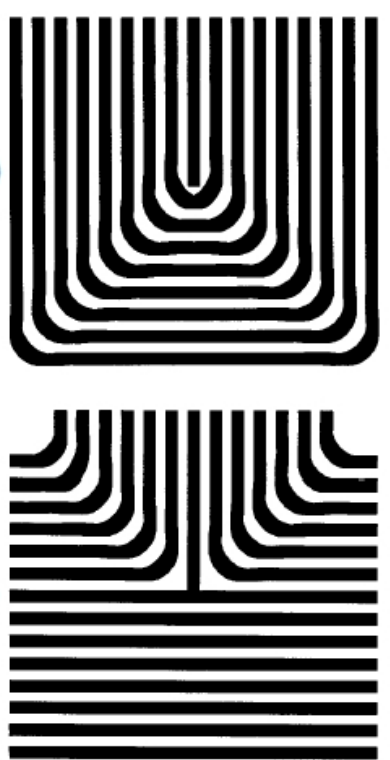

Figura 4.2: Representação esquemática de (a) uma deslocação, (b) e (c) tipos de desclinações que podem ocorrer em fases do tipo faixas. Figura retirada de (34).

de cálculos da energia livre obtida a partir das simulações de Monte Carlo, foram observadas, para $\delta=1$, as fases do tipo faixas e a fase tetragonal com uma transição de primeira ordem entre elas. Os autores ressaltam que apesar de observarem essa transição de primeira ordem para $\delta=1$ em $T=0.4$ a limitação do tamanho da rede $(L=48)$ sugere que esses resultados não sejam tão confiáveis e que devem ser confirmados por outros métodos. Na ref. (12), a partir de simulações de Monte Carlo, são observadas as ordens para a transição da fase do tipo faixas para a de líquido tetragonal. A ordem destas transições em função de $\delta$ está apresentada na tab. 4.2. Em (11) também foi realizado um estudo teórico utilizando a Teoria de Campo Médio. O diagrama de fase teórico difere do obtido por simulações de Monte Carlo (compare as figuras 4.3 e 4.4). O modelo teórico não prevê as fases nemática e tetragonal. Tal divergência deve ser explicada pelo fato de que o método só prevê 


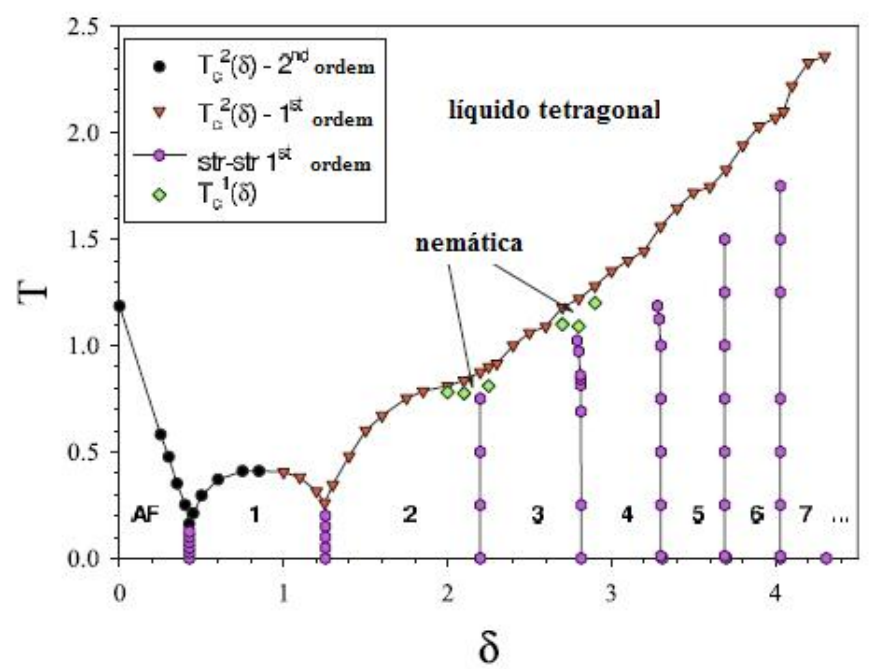

Figura 4.3: Diagrama de fase em função do acoplamento $\delta$ para o modelo de Ising dipolar. Figura adaptada de (11).

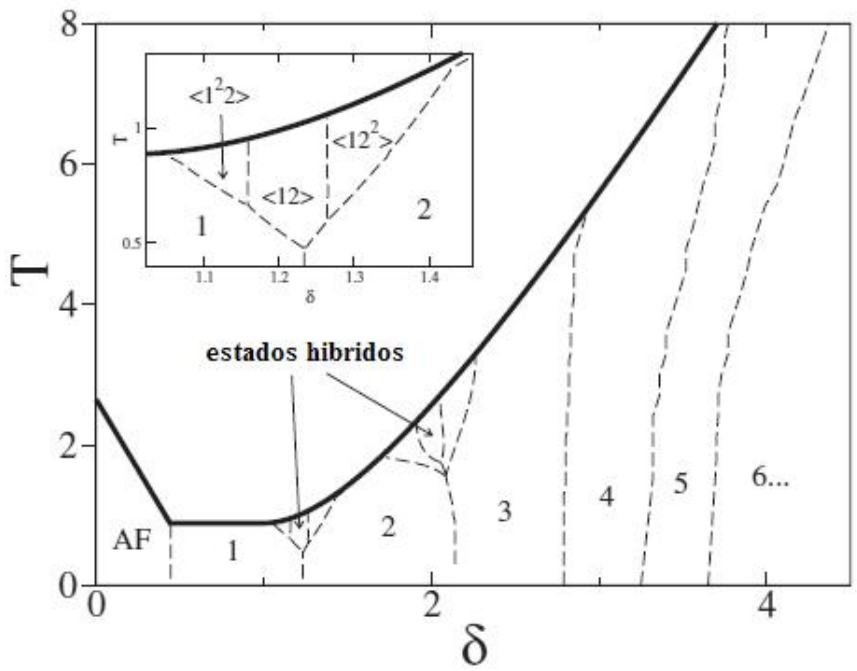

Figura 4.4: Diagrama de fase em função do acoplamento $\delta$ obtido pela Teoria de Campo Médio. Figura retirada de (11).

a fase paramagnética como fase espacialmente desordenada. Entretanto, o modelo prevê soluções híbridas. As regiões de $\delta$ no diagrama de fase onde ocorrem as soluções híbridas coincidem com as regiões em que as simulações numéricas prevêem a fase nemática, com exceção da região entre $h=1$ e $h=2$. Nessa região, as simulações não indicam a presença da fase nemática, entretanto os resultados de campo médio apontam soluções híbridas. Dessa forma, a presença de uma fase nemática nessa 


\begin{tabular}{|c|c|}
\hline$\delta$ & ordem de transição \\
\hline 0.85 & segunda \\
1.7 & primeira \\
2.5 & primeira (fraca) \\
3.0 & segunda \\
\hline
\end{tabular}

Tabela 4.2: Ordem de transição da fase do tipo faixas para a fase tetragonal obtidas na ref. (12) para diferentes valores de $\delta$.

\begin{tabular}{|c|c|c|c|}
\hline$\delta$ & fases observadas & ordem de transição & referência \\
\hline \hline$<0.425$ & anti-ferromagnética $\rightarrow$ tetragonal & contínua & $(9,10,33)$ \\
0.85 & faixas $\rightarrow$ tetragonal & contínua & $(12)$ \\
1.00 & faixas $\rightarrow$ tetragonal & primeira & $(10)$ \\
1.7 & faixas $\rightarrow$ tetragonal & primeira & $(12)$ \\
2.0 & faixas $\rightarrow$ nemática $\rightarrow$ tetragonal & análise não conclusiva e & $(12,11)$ \\
& & primeira, respectivamente & \\
\hline
\end{tabular}

Tabela 4.3: Resumo das transições de fase observadas para alguns acoplamentos $\delta$ conforme obtidas em diferentes trabalhos.

região de acoplamento ainda é obscura.

Os resultados que temos na literatura para o modelo, além de estarem claramente apresentados nas figuras 4.3 e 4.4, também estão resumidos na tabela 4.3 acompanhados das respectivas referências para alguns valores de $\delta$.

A despeito de todo intenso trabalho experimental e teórico, a precisa natureza da ordem das transições entre as fases observadas neste sistema ainda não é bem compreendida e os resultados obtidos não são perfeitamente confiáveis, principalmente aqueles advindos das simulações de Monte Carlo realizadas com redes pequenas. Com nosso trabalho esperamos contribuir para esclarecer o diagrama de fase do modelo na região $\delta \in[0.89,1.30]$. Em específico trabalhamos no intervalo de $\delta$ correspondente a $h=1$ na procura da localização precisa do ponto tricrítico e na obtenção da ordem de transição da fase do tipo faixas para líquido tetragonal. 


\section{Capítulo}

\section{Métodos de Monte Carlo}

\subsection{Introdução}

O método de Monte Carlo (MC) tem a finalidade de construir uma distribuição de microestados característicos (configurações) do sistema descrito por uma hamiltoniana utilizando números (pseudo)-aleatórios. Grandezas físicas são obtidas a partir de médias temporais ${ }^{1}$ envolvendo estas configurações. Esta média estocástica equivale a uma média de ensemble, se o sistema for ergódico. Assim, o tempo desempenha o papel de um rótulo caracterizando os vários estados amostrados e não necessita estar relacionado com o tempo físico (35). As propriedades de um sistema, descritas pela mecânica estatística, é dada de forma não trivial pela presença de interações entre as partículas. Sendo $E(x)$ a energia de uma configuração $x$ (um ponto no espaço de fase do modelo), a média termodinâmica de qualquer observável $O(x)$ é dada por

$$
\langle O\rangle=\frac{\int O(x) \exp [-\beta E(x)]}{\int \exp [-\beta E(x)]} .
$$

A ideia básica do método MC é calcular numericamente as integrais no espaço de fase. Este método foi proposto por Metropolis (36) e é baseado no conceito ideia de amostragem por importância. Aqui, os pontos do espaço de fase não são completamente aleatórios e sim amostrados de acordo com uma probabilidade $\omega(x)$.

\footnotetext{
${ }^{1}$ Neste caso, o tempo corresponde ao número de passos computacionais
} 
Aproximando a integral dada em 5.1 para um somatório, temos:

$$
\langle O\rangle=\frac{\sum_{i=1}^{M} O\left(x_{i}\right) \omega^{-1}\left(x_{i}\right) \exp \left[-\beta E\left(x_{i}\right)\right]}{\sum_{i=1}^{M} \omega^{-1}\left(x_{i}\right) \exp \left[-\beta E\left(x_{i}\right)\right]} .
$$

Escolhendo $\omega\left(x_{i}\right)=\omega_{e q}\left(x_{i}\right) \propto \exp \left[-\beta E\left(x_{i}\right)\right]$, a equação 5.2 se reduz a uma simples média aritmética,

$$
\langle O\rangle=\frac{1}{M} \sum_{i=1}^{M} O\left(x_{i}\right)
$$

A equação 5.3 representa o fato de ser possível construir uma caminhada aleatória de pontos $\left(x_{i}\right)$ através do espaço de fase via um processo markoviano de forma que $\omega\left(x_{i}\right) \rightarrow \omega_{e q}\left(x_{i}\right)$ para $M \rightarrow \infty$. Esse processo markoviano é definido especificando uma probabilidade de transição $W\left(x_{i} \rightarrow x_{i}^{\prime}\right)$ de um ponto no espaço de fase $x_{i}$ para outro $x_{i}^{\prime}$. Para garantir a propriedade de que $\omega\left(x_{i}\right)$ convirja para $\omega_{e q}\left(x_{i}\right)$ é suficiente impor a condição de balanço detalhado

$$
\omega_{e q}\left(x_{i}\right) W\left(x_{i} \rightarrow x_{i}^{\prime}\right)=\omega_{e q}\left(x_{i}^{\prime}\right) W\left(x_{i}^{\prime} \rightarrow x_{i}\right),
$$

o qual implica que a razão entre as probabilidades de transição depende somente da variação na energia, ou seja

$$
\frac{W\left(x_{i} \rightarrow x_{i}^{\prime}\right)}{W\left(x_{i}^{\prime} \rightarrow x_{i}\right)}=e^{-\beta\left[E\left(x_{i}^{\prime}\right)-E\left(x_{i}\right)\right]} .
$$

Consequentemente, podemos escolher a probabilidade de transição entre $x_{i}$ e $x_{i}^{\prime}$ da seguinte forma:

$$
W\left(x_{i} \rightarrow x_{i}^{\prime}\right)= \begin{cases}A^{-1} & , \text { se } E\left(x_{i}^{\prime}\right)<E\left(x_{i}\right) \\ A^{-1} e^{-\beta\left[E\left(x_{i}^{\prime}\right)-E\left(x_{i}\right)\right]} & , \text { se } E\left(x_{i}^{\prime}\right)>E\left(x_{i}\right),\end{cases}
$$

onde $A$ é uma constante qualquer que não afeta o balanço detalhado ${ }^{2}$. A equação 5.6 diz que, se a variação na energia do sistema resultante de uma mudança na configuração de $x_{i} \rightarrow x_{i}^{\prime}$ for negativa, a nova configuração será automaticamente aceita. Do contrário, a nova configuração será aceita com a probabilidadade $e^{-\beta\left[E\left(x_{i}^{\prime}\right)-E\left(x_{i}\right)\right]}$.

\footnotetext{
${ }^{2}$ Usualmente faz-se $A=1$
} 


\subsection{Ensembles generalizados}

A utilização da amostragem por importância escolhendo $\omega(x)$ como o peso de Boltzmann conduz a um algoritmo largamente utilizado. A proposta original com $\omega(x) \propto e^{-\beta E(x)}$ simula o ensemble canônico a uma temperatura fixa $T$ e nos permite calcular grandezas físicas tais como a energia interna em função dessa temperatura. Entretanto, este algoritmo pode ser ineficiente no acesso às possíveis configurações de equilíbrio se existirem grandes barreiras de energia livre entre elas, como por exemplo, quando da ocorrência de transições de fase de primeira ordem. Neste caso, o tamanho da amostragem e/ou o tempo necessário para que se amostre essas configurações se torna muito grande e inviável. Outras escolhas da função peso são possíveis. Na tentativa de se ultrapassar barreiras de energia livre, foi criado o conceito de ensemble generalizado, que fornecem histogramas uniformes na energia. Esses métodos permitem calcular estimar médias canônicas para uma ampla faixa de temperaturas a partir de uma única simulação. A essência dessa aproximação é conseguir amostrar o espaço de estados de um sistema físico de forma que estados particularmente raros, mas importantes, sejam encontrados com frequência.

Para os algoritmos generalizados, um movimento do microestado $x$ para $x^{\prime}$ é aceito com a probabilidade

$$
W\left(x \rightarrow x^{\prime}\right)=\min \left[1, \omega\left(x^{\prime}\right) / \omega(x)\right]
$$

A escolha usual da amostragem por importância leva a um histograma $H(E)$ com distribuição canônica $H(E) \propto \rho(E) e^{-\beta E}$ (onde $\rho(E)$ é a densidade de estados). A escolha $\omega(E) \propto 1 / \rho(E)$ leva a um histograma uniforme e é utilizada por algoritmos tais como os algoritmos multicanônico (23), entrópico (37) e de Wang-Landau (38). Utilizamos neste trabalho o algoritmo multicanônico. Embora as discussões presentes na literatura $(39,40)$ mostrem a equivalência entre os algoritmos entrópico e multicanônico, apresentaremos também a formulação do algoritmo entrópico com o intuito de facilitar o entendimento da formulação do algoritmo multicanônico. 


\subsubsection{O algoritmo entrópico}

Em 1993, posteriormente à apresentação do algoritmo multicanônico por Berg e Neuhaus (23) (que veremos na seção 5.2.2), Jooyoung Lee (37) apresentou o algoritmo entrópico como um novo algoritmo de Monte Carlo com a justificativa de que a amostragem entrópica é um método geral e que qualquer tipo de amostragem é uma particularidade do método proposto. Observando que a função de partição pode ser escrita como

$$
Z(\beta)=\sum_{E} \rho(E) e^{-\beta E}=\sum_{E} \exp [S(E)-\beta E],
$$

onde $S(E)$ é a definção da entropia microcanônica de Boltzmann. Podemos obter uma distribuição arbitrária $\omega(E) \propto \exp [S(E)-J(E)]$ impondo que

$$
\frac{W\left(x \rightarrow x^{\prime}\right)}{W\left(x^{\prime} \rightarrow x\right)}=e^{-\left[J\left(E\left(x^{\prime}\right)\right)-J(E(x))\right]} .
$$

Note que a amostragem por importância convencional nada mais é que o caso particular com $J(E)=\beta E$. Como veremos, o algoritmo multicanônico pode ser considerado dessa forma como um caso particular com $J(E)=b(E) E-a(E)$. O objetivo do algoritmo entrópico é obter a densidade de estados do sistema ou a entropia microcanônica $S(E)$ utilizando o peso $J(E)$. Se $J(E)=S(E)$, a produção fornecerá uma distribuição aproximadamente uniforme sobre todo o intervalo de energia explorado. Dessa forma, é necessário, antes de realizar uma produção com $\omega(E)=e^{-S(E)}$, obter uma boa estimativa para $S(E)$. Essa estimativa é obtida a partir dos seguintes passos:

i) inicialmente, faça $J(E)=S(E)=0$ para todo $E$;

ii) obtenha o histograma $H(E)$ de acordo com a equação 5.9;

iii) uma nova estimativa para $S(E)$ é dada por

$$
S(E)= \begin{cases}J(E) & , \text { se } H(E)=0 \\ J(E)=J(E)+\ln H(E) & , \text { caso contrário }\end{cases}
$$

Os passos ii e iii são repetidos até que o sistema amostre todo o intervalo desejado para a energia interna $E$. 


\subsubsection{O algoritmo multicanônico}

No algoritmo multicanônico o peso $\omega(E) \propto e^{-J(E)}$ é escrito em função de dois parâmetros livres $a(E)$ e $b(E)$, denominados parâmetros multicanônicos, da seguinte forma:

$$
\omega_{1 / \rho(E)}=\frac{1}{\rho(E)}=e^{-S(E)}=e^{-b(E) E+a(E)} .
$$

Como uma extensão da terminologia microcanônica, podemos chamar $b(E)$ de inverso da temperatura microcanônica e $a(E)$ de energia livre microcanônica adimensional. Uma simulação multicanônica fornece, da mesma forma que o algoritmo entrópico, a entropia microcanônica pois os parâmetros multicanônicos procurados fornecem $\omega_{m u}(E)=\omega_{1 / \rho(E)}=\frac{1}{\rho(E)}$. Dessa forma, a entropia microcanônica é obtida a partir de $S(E)=-\ln \rho(E)=b(E) E-a(E)$.

Uma simulação canônica convencional calcula valores esperados na temperatura $T$ e pode, por técnicas de repesagem, somente extrapolar esses valores esperados para a vicinidade dessa temperatura. Em contraste, uma única simulação multicanônica nos permite obter propriedades de equilíbrio no ensemble de Gibbs em qualquer temperatura. De forma análoga ao algoritmo entrópico, o algoritmo multicanônico requer as mesmas duas etapas:

i) obter uma estimativa para $\omega_{m u}(E)$ a partir dos parâmetros multicanônicos $a(E)$ e $b(E)$ para todo $E$;

ii) produzir uma simulação de Monte Carlo com o peso final fixo $\omega_{m u}(E)$.

A partir do momento em que a primeira etapa da simulação multicanônica é efetuada, todas as propriedades termodinâmicas do sistema são obtidas na segunda etapa.

\subsubsection{Estimativas canônicas}

Seguindo o algoritmo multicanônico, a distribuição de configurações corresponde à probabilidade multicanônica é dada por

$$
p_{m u}(E)=c_{m u} \rho(E) \omega_{m u}(E)
$$


onde $c_{m u}=1 / \sum_{E} \rho(E) \omega_{m u}(E)$ para garantir que $\sum_{E} p_{m u}=1$. Lembrando que $\rho(E)=H_{m u}(E) \omega_{m u}(E)^{-1}$, onde $H_{m u}(E)$ é o histograma de energia obtido a partir de uma simulação multicanônica, a equação 5.12 também pode ser escrita para fins práticos $^{3}$ da seguinte forma:

$$
p_{m u}(E)=c_{m u} H_{m u}(E),
$$

Se os parâmetros multicanônicos fornecerem valores apropriados para a entropia microcanônica, então a aproximação

$$
\rho(E) \approx 1 / \omega_{m u}(E)
$$

é válida. Substituindo 5.14 em 5.12, podemos verificar que o histograma produzido deve ser aproximadamente uniforme, pois

$$
p_{m u}(E) \approx c_{m u}
$$

A probabilidade canônica é dada por

$$
p_{\beta}(E)=c_{\beta} \rho(E) e^{-\beta E}
$$

onde a constante $c_{\beta}=1 / Z(\beta)$ é necessária para garantir que $\sum_{E} p_{\beta}(E)=1$. Substituindo a densidade de estados dada em 5.14 na equação 5.16, temos a repesagem da probabilidade $p_{\beta}(E)$ para o ensemble canônico,

$$
p_{\beta}(E)=\frac{c_{\beta}}{c_{m u}} \frac{p_{m u}(E)}{\omega_{m u}(E)} e^{-\beta E}=c_{\beta} \frac{H_{m u}(E)}{\omega_{m u}(E)} e^{-\beta E} .
$$

Também podemos utilizar a aproximação 5.11 e estimar a probabilidade canônica como

$$
p_{\beta}(E) \approx c_{\beta} \omega_{m u}(E)^{-1} e^{-\beta E}
$$

Consequentemente, construímos a função de partição canônica a partir dos histogramas produzidos e dos parâmetros multicanônicos da seguinte forma:

$$
Z(\beta)=\sum_{E} H_{m u}(E) \exp [-\beta E+b(E) E-a(E)]
$$

\footnotetext{
${ }^{3}$ As simulações fornecem o histograma $H_{m u}(E)$ a partir do peso multicanônico $\omega_{m u}(E)$
} 
Assim, grandezas importantes no ensemble canônico, como a energia livre de Helmholtz,

$$
F(\beta)=-\beta^{-1} \ln Z(\beta),
$$

e a entropia

$$
S(\beta)=\beta(E-F),
$$

podem ser estimadas.

O valor esperado $\langle O\rangle$ a uma dada temperatura $T$, sendo $O$ função da energia interna $E$, é dado por

$$
\langle O\rangle(\beta)=\frac{\sum_{E} O(E) H(E) \exp [-\beta E]}{\sum_{E} H(E) \exp [-\beta E]},
$$

onde $H(E)$ o histograma de energia produzido. Na presente simulação utilizamos o histograma $H_{m u}(E)$, e o estimador multicanônico de $\langle O\rangle$ é dado por

$$
\langle O\rangle(\beta)=\frac{\sum_{E} O(E) H_{m u}(E) \exp [-\beta E+b(E) E-a(E)]}{\sum_{E} H_{m u}(E) \exp [-\beta E+b(E) E-a(E)]},
$$

onde as somas são efetuadas sobre todos os valores de $E$ para os quais $H_{m u}(E)$ tem valores não nulos.

\subsubsection{Relações de recorrência para obtenção do peso multicanônico}

A primeira etapa do algorítimo é sem dúvida a mais dispendiosa pois consiste na realização de diversas repetições até que todo o intervalo de energia desejado seja amostrado e de forma uniforme. Para tanto, são utilizadas relações recursivas para os parâmetros multicanônicos. O índice $n$ no peso multicanônico $\omega_{m u}^{n}(E)$ e nos parâmetros $a^{n}(E)$ e $b^{n}(E)$, será utilizado a partir de agora para associá-lo com a $n$-ésima repetição. A simulação é iniciada $\operatorname{com} \omega_{m u}^{0}(E)=1$ para todo $E$, que consiste em uma simulação a $T$ infinito, pois $b(E)=a(E)=0$. Os passos subsequentes são realizados para obter melhores estimativas possíveis para $\omega_{m u}^{n}(E)$, com $n=1,2,3, \ldots$ para todo $E$.

Como já comentado, o parâmetro multicanônico $b(E)$ está relacionado com o inverso da temperatura microcanônica. Da relação termodinâmica $F=E-T S$ 
fazemos a associação $b(E)=1 / T(E)$ e $a(E)=F(E) / T(E)$. Assim, por definição, a temperatura microcanônica é

$$
b(E)=\frac{1}{T(E)}=\frac{\partial S(E)}{\partial E} .
$$

Para energias discretizadas podemos escrever

$$
b(E)=[S(E+\epsilon)-S(E)] / \epsilon .
$$

A identidade $S(E)=b(E) E-a(E)$ implica que

$$
S(E)-S(E-\epsilon)=b(E) E-b(E-\epsilon)(E-\epsilon)-a(E)+a(E-\epsilon) .
$$

Fazendo $S(E)-S(E-\epsilon)=\epsilon b(E-\epsilon)$ temos

$$
a(E-\epsilon)=a(E)+[b(E-\epsilon)-b(E)] E .
$$

Note que, definindo um valor para $a\left(E_{\max }\right), a(E)$ é obtido de forma recursiva a partir do momento que se tem $b(E)$. Os valores de $a^{n}(E)$ dependem apenas de $b^{n}(E)$ na $n$-ésima repetição, ou seja,

$$
a^{n}(E-\epsilon)=a^{n}(E)+\left[b^{n}(E-\epsilon)-b^{n}(E)\right] E .
$$

Falta agora definir como obter os valores para $b^{n+1}(E)$ a partir da $n$-ésima repetição.

O histograma de energias da $n$-ésima repetição é dado por $H_{m u}^{n}(E)$. Para evitar que $H_{m u}^{n}(E)=0$, o substituímos por

$$
H_{m u}^{n}(E)=\max \left[H_{0}, H_{m u}^{n}(E)\right]
$$

onde $H_{0}$ é um número entre 0 e 1 . Definimos

$$
\omega_{m u}^{n+1}(E)=e^{-S^{n+1}(E)}=c \frac{\omega_{m u}^{n}(E)}{H_{m u}^{n}(E)},
$$

onde o valor da constante $c$ é irrelevante para o processo de amostragem. Assim, um estimador da entropia microcanônica é definido como

$$
S^{n+1}(E)=-\ln c+S^{n}(E)+\ln H_{m u}^{n}(E)
$$


Inserindo esta relação em 5.25 obtemos finalmente a seguinte relação para os $b^{n}(E)$,

$$
b^{n+1}(E)=b^{n}(E)+\left[\ln H_{m u}^{n}(E+\epsilon)-\ln H_{m u}^{n}(E)\right] / \epsilon .
$$

Entretanto, é conveniente utilizar a relação introduzida em $(41,26,8)$ onde se define o peso normalizado

$$
\hat{g}_{0}^{n}(E)=\frac{g_{0}^{n}(E)}{g^{n}(E)+g_{0}^{n}(E)},
$$

Os pesos $g^{n}(E)$ são obtidos pela relação $g^{n+1}(E)=g^{n}(E)+g_{0}^{n}(E), \operatorname{com} g^{0}(E)=0$. A partir dos pesos $g_{0}^{n}(E)$ são definidos por

$$
g_{0}^{n}(E)=\frac{H_{m u}^{n}(E+\epsilon) H_{m u}^{n}(E)}{H_{m u}^{n}(E+\epsilon)+H_{m u}^{n}(E)} .
$$

A relação recursiva dada em 5.31 é reescrita na nova forma como

$$
b^{n+1}(E)=b^{n}(E)+\hat{g}_{0}^{n}(E)\left[\ln H_{m u}^{n}(E+\epsilon)-\ln H_{m u}^{n}(E)\right] / \epsilon .
$$

Aqui nós vimos como as atualizações dos parâmetros multicanônicos são realizadas. Entretanto, não mencionamos quando atualizar esses parâmetros. ou seja, após quantos passos de Monte Carlo devemos terminar uma repetição e começar outra, de forma a obter $b^{n+1}(E)$ e $a^{n+1}(E)$ a partir de $b^{n}(E)$ e $a^{n}(E)$. Veremos no próximo capítulo que o número de passos de Monte Carlo de cada repetição dependerá do número de round trips, que definiremos a seguir. 


\section{CAPÍtulo}

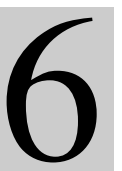

\section{SimUlaÇÃO DO MODELO}

Neste capítulo apresentaremos detalhes do processo de simulação do modelo de Ising dipolar, abordando os métodos utilizados na produção e análise dos dados. Como já visto, o algoritmo multicanônico requer a obtenção dos parâmetros multicanônicos para definir a probabilidade de amostragem das possíveis configurações. Dessa forma, apresentaremos os critérios utilizados para obter esses parâmetros: o número de passos de Monte Carlo e de round trips.

As simulações fornecem a densidade de estado do sistema, a qual é utilizada para obter a função de partição canônica. Os zeros complexos da função de partição canônica foram calculados de duas maneiras: para redes $L=12,20$ e 32 calculamos os zeros de um polinômio lançando mão do aplicativo Mathematica. Para $L>32$ foi utilizado outro método, o qual consiste na separação da função de partição em duas partes: uma parte imaginária e uma parte real. Após isso, é efetuado um escaneamento nas duas partes em busca dos respectivos zeros. Os zeros complexos da função de partição correspondem às soluções simultâneas das suas partes real e imaginária. Em termos gráficos, isso equivale a encontrar os pontos dos gráficos onde os zeros dos termos imaginário e real se cruzam. Cada zero obtido por esse método é então refinado fazendo uso da rotina de otimização Amoeba (42), que necessita de um valor de entrada bem próximo do zero para convergir. Após a obtenção do zero mais próximo do eixo real $u_{1}^{0}(L)$, a ordem da transição é definida pelo expoente $\nu$ a partir das relações de escala para esses zeros.

O processo de obtenção dos resultados consiste das seguintes etapas: 
- obtenção dos parâmetros multicanônicos;

- produção das séries por meio do algoritmo multicanônico;

- obtenção do primeiro zero da função de partição para cada série produzida;

- cálculo dos zeros e de seus erros a partir das séries produzidas;

- estimativa do expoente $\nu$ a partir da relação de escala para o primeiro zero da função de partição e determinação da ordem da transição de fase do tipo faixas para a fase de líquido tetragonal;

- obtenção da temperatura crítica e construção do diagrama de fase.

\subsection{Condições periódicas de contorno e o método da soma de Ewald}

Por causa do caráter de longo alcance do modelo de Ising dipolar as condições de contorno não devem ser restritas somente ao contorno $L+1$ e $L-1$ de uma rede quadrada de volume $L \times L$, onde os spins localizados em $(i, L)$ interagem com os spins localizados em $(i, 1)$ e os spins localizados em $(L, i)$ interagem com os spins $(1, i)$, sendo $i=1,2, \ldots, L$. Esse tipo de condição de contorno é aplicado somente no termo de interação de troca entre primeiros vizinhos. Já para o termo dipolar, utilizamos imagens replicadas infinitamente no plano, onde o momento de dipolo de cada partícula deve interagir com os momentos de dipolo de todas as outras partículas, inclusive com as imagens dessas partículas e com a sua própria imagem. A figura 6.1 representa as condições de contorno periódicas para interações entre primeiros vizinhos (caixas em azul) e as interações dipolares (setas). A seta na cor preta representa uma interação entre a partícula $i$ e a partícula $j$ contida dentro da rede quadrada $L \times L$ (em branco). As setas azul e verde indicam, respectivamente, interações entre a partícula $i$ e as partículas $j^{\prime}$ (imagem da partícula $j$ ) e $j^{\prime \prime}$ (outra imagem da partícula $j$ ). O termo dipolar da energia dado pela equação 4.5, considerando as interações de cada partícula com todas as outras e suas imagens, 


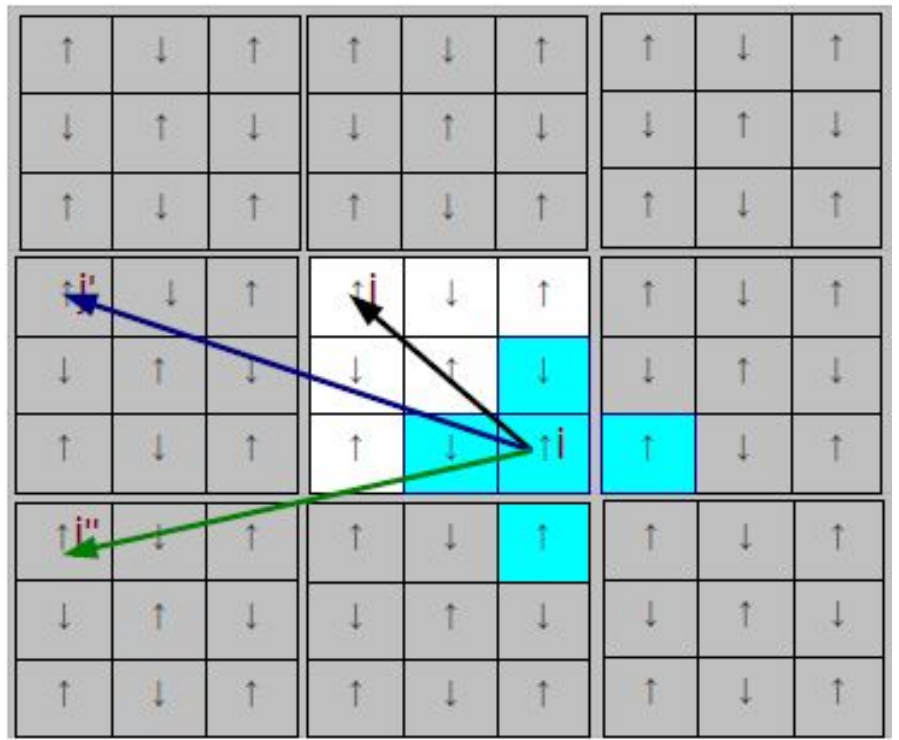

Figura 6.1: Representação das condições periódicas de contorno para interações de troca entre uma partícula $i$ e seus primeiros vizinhos (caixas na cor azul) e interações dipolares (setas) que ocorrem entre todas as outras partículas e suas respectivas imagens.

além de sua própria imagem, fica escrito como

$$
E_{\text {dipolar }}=\frac{1}{2} \sum_{i=1}^{N} \sum_{j=1}^{N} \sum_{|\mathbf{n}|=0}^{\infty}, \frac{\sigma_{i} \sigma_{j}}{\left|\mathbf{r}_{\mathbf{i j}}+\mathbf{n}\right|^{3}},
$$

onde' indica que o vetor $\vec{n}=0$ é excluído do somatório quando $i=j$.

Temos dessa forma dois tipos de condições periódicas de contorno: uma para interações de troca entre primeiros vizinhos e outra para interações dipolares entre

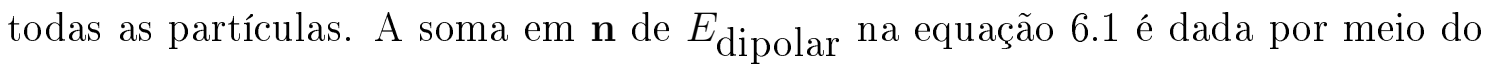
método da soma de Ewald, que converte este somatório, de convergência lenta, em três outros somatórios que convergem mais rapidamente. Esses três termos são os seguintes: um termo no espaço real, um termo no espaço recíproco e um termo de auto-interação. Utilizando o método da soma de Ewald temos uma nova expressão para a energia dipolar:

$$
E_{\text {dipolar }}=E_{\text {real }}+E_{\text {recíproco }}+E_{\text {auto-interação }}
$$


com

$$
\begin{array}{ll}
E_{\text {real }} & =\frac{1}{2} \sum_{i=1}^{N} \sum_{j=1}^{N} \sum_{|\mathbf{n}|=0}^{\infty} \sigma_{i} \sigma_{j}\left[\frac{\operatorname{erfc}\left(\alpha\left|\mathbf{r}_{\mathbf{i j}}+\mathbf{n}\right|\right)}{\left|\mathbf{r}_{\mathbf{i j}}+\mathbf{n}\right|^{3}}+\frac{2 \alpha}{\sqrt{\pi}} \frac{e^{-\alpha^{2}\left|\mathbf{r}_{\mathbf{i j}}+\mathbf{n}\right|^{2}}}{\left|\mathbf{r}_{\mathbf{i j}}+\mathbf{n}\right|^{2}}\right], \\
E_{\text {recíproco }} & =\frac{\pi}{L^{2}} \sum_{i=1}^{N} \sum_{j=1}^{N} \sum_{|\mathbf{G}|=0}^{\infty} \sigma_{i} \sigma_{j}\left[\frac{2 \alpha}{\sqrt{\pi}} e^{\frac{-G^{2}}{4 a^{2}}}-\operatorname{Gerfc}\left(\frac{G}{2 \alpha}\right)\right] \cos \left(\mathbf{G} \cdot \mathbf{r}_{\mathbf{i j}}\right), \\
E_{\text {auto-interação }} & =-\frac{2 \alpha^{3}}{3 \sqrt{\pi}} \sum_{i=1}^{N} \sigma_{i}^{2},
\end{array}
$$

onde o vetor $\mathbf{G}=2 \pi\left(k_{1} \mathbf{e}_{\mathbf{1}}+k_{2} \mathbf{e}_{\mathbf{2}}\right) / L$ com $k_{1}$ e $k_{2}$ inteiros.

Todo o desenvolvimento referente às condições de contorno periódicas já foi discutido na dissertação de mestrado de Leandro G. Rizzi, que também estudou o modelo de Ising dipolar. Maiores detalhes sobre o método vide (8). No estudo desenvolvido sobre a convergência das somas de Ewald, foi observado no trabalho citado acima, que, para este modelo, o parâmetro $\alpha$ deve ser igual a 3.5 em todas as simulações. A interação de longo alcance faz com que as simulações sejam demoradas, sendo que a maior parte do tempo é utilizada na etapa de obtenção dos parâmetros multicanônicos. Foi necessário então limitar o tamanho das redes utilizadas ao valor máximo de $L=64$.

\subsection{Simulações multicanônicas}

Foram geradas séries temporais utilizando o algoritmo multicanônico para redes de tamanho $L=12,20,32$, 48 e 64 . As séries produzidas foram iniciadas a partir de configurações aleatórias. A energia mínima por spin $e_{0}=E_{0} / N$ foi escolhida com base na obtenção teórica da energia do estado fundamental para $L=128$. Para isso, calculamos as energias dos estados do tipo faixa com $h=1,2$ e 3 em função do acoplamento $\delta$ (veja a figura 6.2). Verificamos qual é a configuração de menor energia em função de $\delta$. Note que, para $\delta<1.2585$, o estado com largura de faixas $h=1$ é o de menor energia, com $e_{0}=-0.4677$. Já para o intervalo $\delta \in[1.2585,2.2017]$, as menores energias ocorrem na configuração do tipo faixas $h=2$ e para o intervalo $\delta \in[2.2017,2.6]$, ocorrem $\operatorname{com} h=3$. A tabela 6.1 mostra a dependência entre a largura das faixas $h$ e o acoplamento $\delta$ para $L=128$ com base nesse cálculo. Assim, 


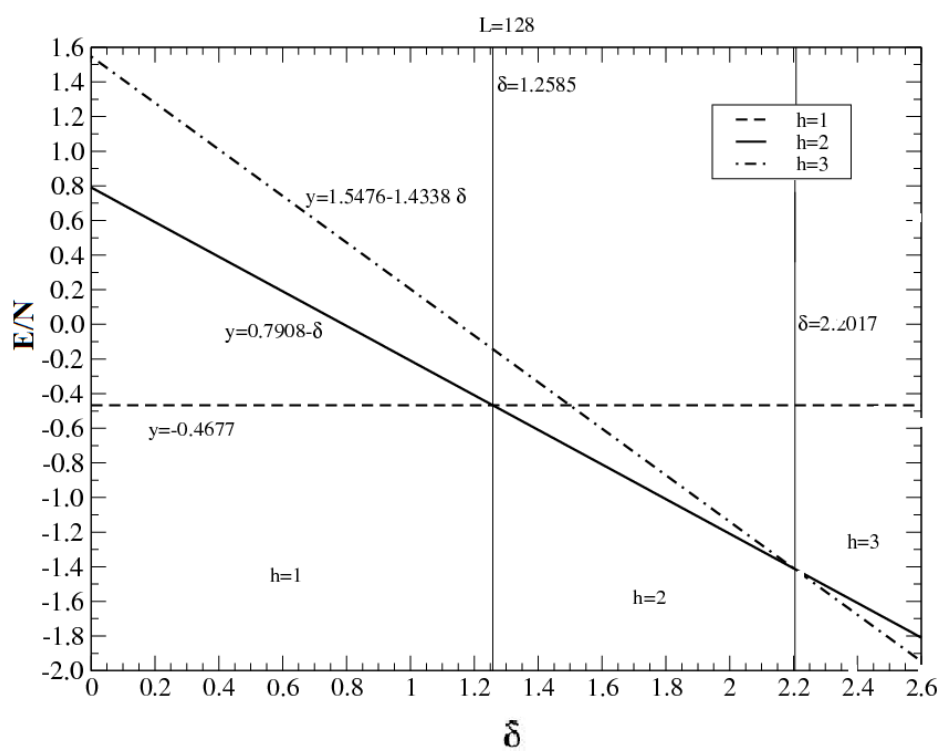

Figura 6.2: Energias calculadas para os estados do tipo faixas $h=1,2$ e 3 em função de $\delta$ para $L=128$.

no intervalo em que trabalhamos encontramos o estado fundamental do tipo faixas $h=1$, com exceção do $\delta=1.30$, que já apresenta o estado do tipo faixas $h=2$.

Para a construção dos histogramas de energia tivemos que dividir as energias do sistema em pequenos intervalos de largura $\epsilon$. Assim, a energia característica de cada intervalo de largura $\epsilon=1$ na energia total (ou $\epsilon / L^{2}$ em energia por spin), é dada pela relação

$$
E_{m}=e_{0} L^{2}+m \epsilon
$$

sendo que $m=0,1,2, \ldots, L^{2}$. Dessa forma, a energia mínima total do sistema é dada por $e_{0} L^{2}$. Consequentemente, os parâmetros multicanônicos obtidos e usados na simulação estão relacionados a um determinado intervalo de energia, ou seja $a(E(m))$ e $b(E(m))$ correspondem na verdade a um intervalo de energia discretizado, caracterizado por $m$. Para a determinação dos parâmetros multicanônicos para $L=12$ e $L=20$ inicialmente utilizamos $\epsilon=0.5$ com o objetivo de termos mais pontos no gráfico de $b(E)$ e $a(E)$. Entretanto, observamos vários intervalos de energia de largura $\epsilon$ vazios, ou seja, nenhuma configuração foi amostrada em alguns desses intervalos. Assim, decidimos utilizar como padrão $\epsilon=1$. 


\begin{tabular}{ccc}
\hline$h$ & $\delta$ & $e_{0}$ \\
\hline 1 & $0.44-1.2585$ & -0.4677 \\
2 & $1.2585-2.2017$ & $0.7908-\delta$ \\
3 & $2.2017-2.81$ & $1.5476-1.4338 \delta$ \\
\hline
\end{tabular}

Tabela 6.1: Relação entre $\delta, h$ e energia do estado fundamental utilizada nas simulações.

\begin{tabular}{ccc}
\hline$m$ & \multicolumn{2}{c}{ histograma } \\
\hline & $\epsilon=0.5$ & $\epsilon=1$ \\
\hline 2 & 0 & 282 \\
3 & 0 & 0 \\
4 & 159 & 23292 \\
5 & 0 & 28093 \\
6 & 0 & 25084 \\
7 & 10756 & 23528 \\
8 & 13795 & 23379 \\
\hline
\end{tabular}

Tabela 6.2: Histograma para baixas energias com $L=12$ para $\epsilon=0.5$ e $\epsilon=1$.

O procedimento adotado desde a obtenção dos parâmetros multicanônicos até a produção de dados foi o seguinte:

- Obtenção dos parâmetros multicanônicos $a^{n}(E)$ e $b^{n}(E)$ :

parte A - cobrindo todo o intervalo de energia:

Os parâmetros multicanônicos $a^{n+1}(E)$ e $b^{n+1}(E)$ são atualizados a partir do histograma $H_{m u}^{n}$ produzido a cada 3 idas e voltas entre as energias máxima e mínima amostradas ${ }^{1}$ ou até atingir um número máximo de passos de $M C$ $\left(\max _{M C}\right)$. Chamaremos esse processo do sistema ir da maior para a menor energia $m_{\min }$ amostrada de round trip $(R T)$, termo comumente utilizado em inglês. O número máximo $\operatorname{nmax}_{M C}$ é definido $\operatorname{como}^{2} \operatorname{nmax}_{M C}=3\left\langle n_{M C}\right\rangle$.

\footnotetext{
${ }^{1}$ É suficiente que sejam contadas as idas e voltas entre a energia mediana e a mínima.

${ }^{2} \mathrm{O}$ valor $\left\langle n_{M C}\right\rangle$ é obtido somando o $n_{M C}$ a cada atualização dos parâmetros multicanônicos e
} 
Como na primeira atualização não temos uma energia mínima amostrada, esta energia mínima é determinada após 50000 passos de $M C$ (cada passo de $M C$ consiste de $N=L^{2}$ varreduras). A menor energia amostrada em cada atualização é mantida pelo programa em uma variável que chamamos de $m_{\text {min }}$ e é utilizada na próxima atualização para contar o número de $R T$. Esse procedimento é repetido até que o sistema consiga chegar à energia do estado fundamental.

parte B - definindo o peso multicanônico:

Depois que obtivemos o peso multicanônico para todas as energias, uma nova atualização é feita. Essa nova atualização exige um maior número de $R T$ (utilizamos $20 R T$ ) com o intuito de obter parâmetros multicanônicos melhores, para a definição do peso $\omega(E)=e^{a(E)-b(E) E}$. O número de passos de $M C\left(n_{M C}\right)$ necessários para essa etapa é guardado para ser utilizado na etapa a seguir.

- Produção de dados: com o peso multicanônico definido, produzimos 16 séries com $n_{M C}$ de forma a termos em torno de $20 R T$ por série. Os $n_{M C}$ utilizados podem ser vistos na tabela 6.3. As densidades de estados fornecidas por essas séries são utilizadas na construção da função de partição canônica para a obtenção de seus zeros e as correspondentes estimativas do erro estatístico.

Como estamos interessados em encontrar o ponto tricrítico, a ordem da transição de fase foi obtida para diferentes valores de $\delta$ e dessa forma os resultados serão apresentados para cada um destes. Entretanto, somente para fins ilustrativos, mostramos o processo de atualização dos parâmetros multicanônicos para $\delta=0.89$. $\mathrm{Na}$ figura 6.3 temos os histogramas para diferentes repetições $n$ de atualização desses parâmetros multicanônicos para todos os valores de $L$ utilizados. Note que os histogramas tornam-se aproximadamente uniformes e amostram um intervalo maior de energias à medida que se atualiza os parâmetros $a^{n}(E)$ e $b^{n}(E)$.

Os parâmetros multicanônicos finais utilizados para definir o peso multicanônico são mostrados no apêndice A para todos os valores de $L$ e $\delta$ simulados. Aqui, para dividindo pelo total de atualizações feitas até o momento 


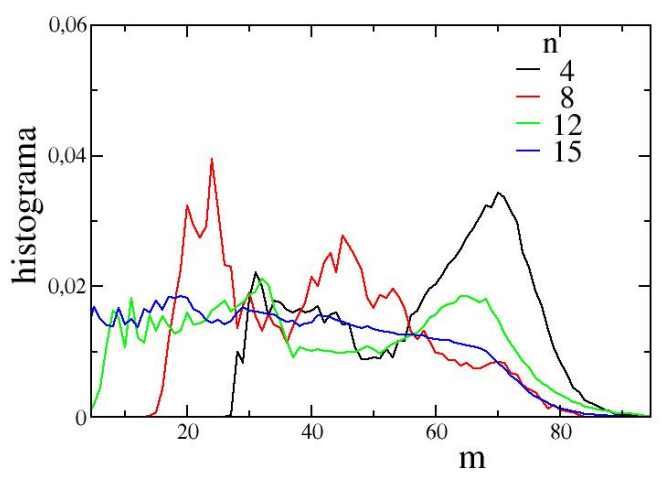

(a) $L=12$

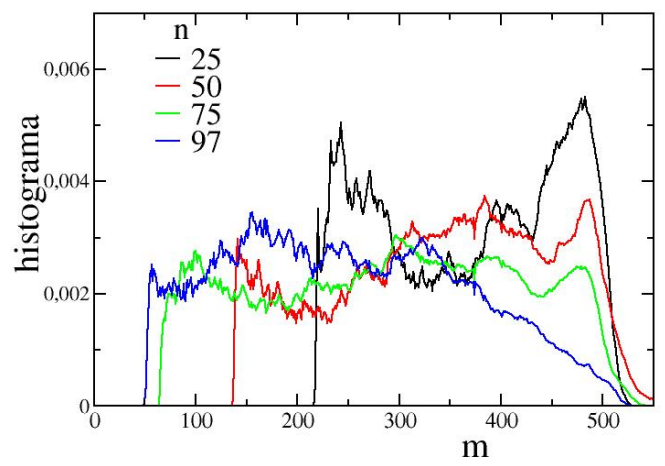

(c) $L=32$

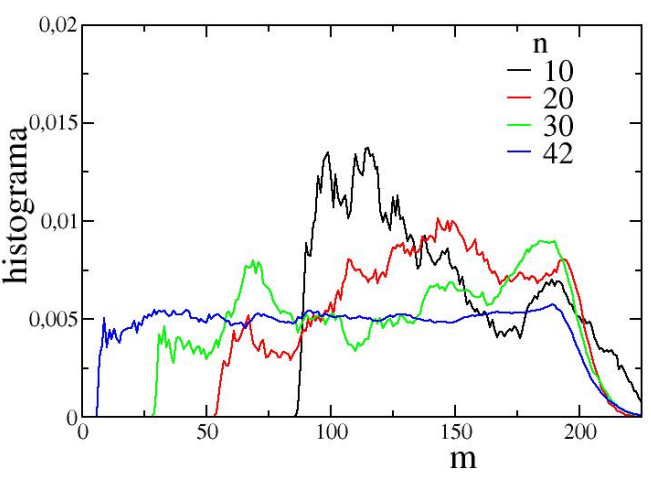

(b) $L=20$

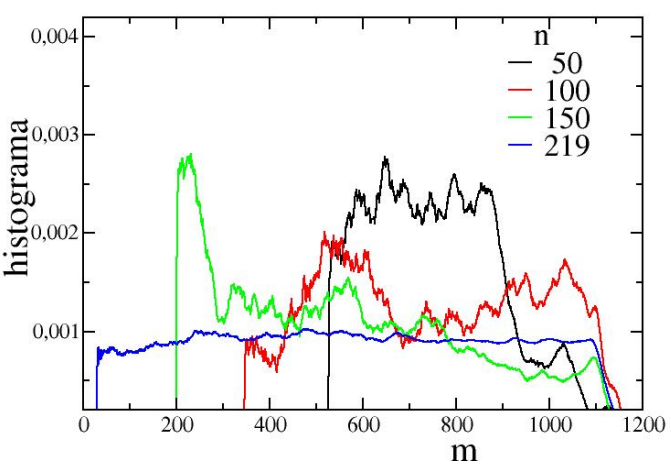

(d) $L=48$

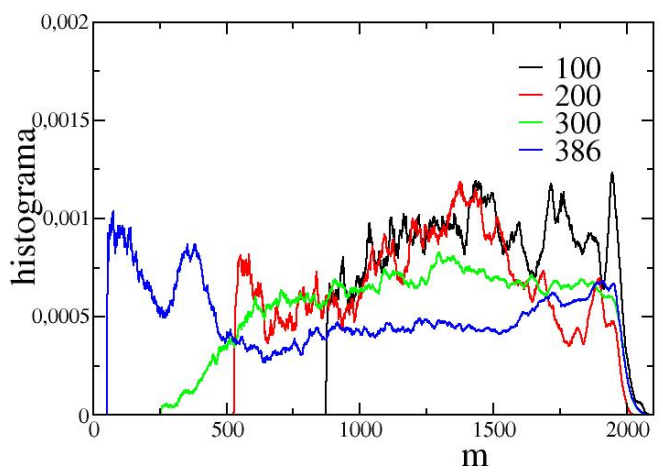

(e) $L=64$

Figura 6.3: Histogramas de energia produzidos em diferentes repetições $n$ para atualização dos parâmetros multicanônicos $a^{n}(E)$ e $b^{n}(E)$ para $\delta=0.89$. 


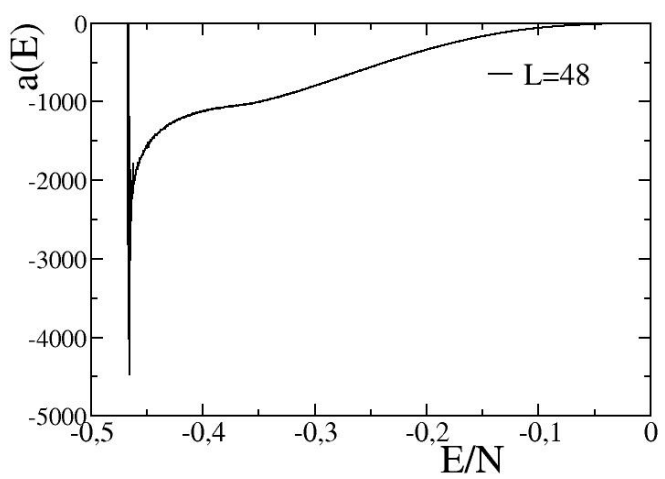

(a) $\delta=0.89$

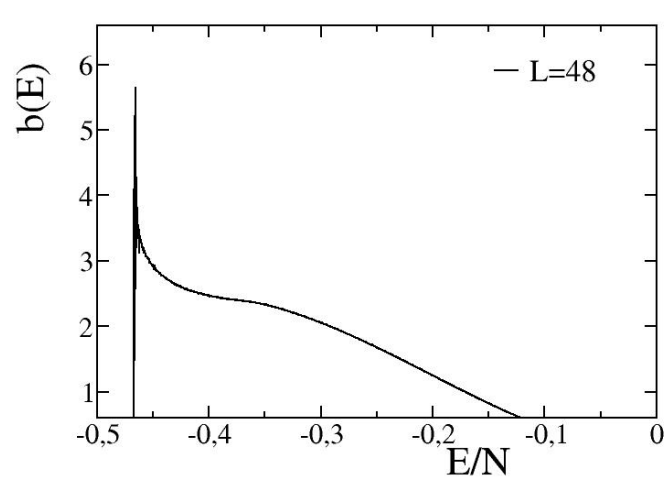

(b) $\delta=0.89$

Figura 6.4: Parâmetros multicanônicos finais utilizados para a produção de dados para $L=48$ : (a) $a(E)$ e (b) $b(E)$.

\begin{tabular}{rrrrrrrrrrr}
$L \backslash \delta$ & 0.89 & 0.91 & 0.93 & 0.95 & 0.97 & 1.00 & 1.10 & 1.20 & 1.30 \\
\hline 12 & 11632 & 12032 & 14026 & 10186 & 16893 & 12279 & 24216 & 51171 & 73362 \\
20 & 47290 & 52098 & 46969 & 50127 & 78380 & 63333 & 91158 & 223934 & 269598 \\
32 & 188620 & 149412 & 105008 & 158214 & 213036 & 179126 & 236436 & 959554 & 1354207 \\
48 & 483287 & 380451 & 645854 & 503469 & 484012 & 582030 & 879740 & 2285886 & 3256588 \\
64 & 824022 & 938475 & 751156 & 1104830 & 1300445 & 1296208 & 2281061 & 5579023 & - \\
\hline
\end{tabular}

Tabela 6.3: $n_{M C}$ utilizados na produção de dados.

fins ilustrativos mostramos esses parâmetros finais para $\delta=0.89$ correspondentes às simulações com redes $L=48$ (figura 6.4).

\subsection{Zeros da função de partição canônica}

A partir dos histogramas produzidos $H_{m u}(E)$ e do peso multicanônico, obtemos a densidade de estados do sistema,

$$
\rho(E) \propto \frac{H_{m u}(E)}{w_{m u}(E)} .
$$

Por sua vez, tendo a densidade de estados, podemos construir a função de partição canônica,

$$
Z(E)=\sum_{E} \rho(E) \exp [-\beta(E)]
$$


Considerando o processo de discretização introduzido introduzido na equação 6.3, $E_{m}=e_{0} L^{2}+m \epsilon$, a equação anterior pode ser escrita como

$$
Z(m)=e^{-\beta e_{0} L^{2}} \sum_{m=0} \rho(m) \exp (-\beta m \epsilon)
$$

Como $\epsilon=1$, os zeros da função de partição são os mesmos do polinômio

$$
Z(m)=\sum_{m=0} \rho(m) u^{m}
$$

onde $u=e^{-\beta}$. Entretanto, por razões numéricas, a obtenção dos zeros com a função de partição escrita nessa forma polinomial somente é viável até $L=32$. Para redes maiores a densidade de estados assume valores além da precisão da máquina, ou seja, valores muito pequenos passam a ser arredondados para zero e valores muito grandes estão além da capacidade da representação numérica. Para $L>32$ os zeros da função de partição foram procurados separadamente nas partes $\operatorname{Re}(Z)$ e $\operatorname{Im}(Z)$ da função de partição. Um zero da função de partição é obtido quando a linha de zeros de $\operatorname{Re}(Z)$ e de $\operatorname{Im}(Z)$ se cruzam. Na figura 6.5 temos a ilustração dos zeros em $\beta$, também para $L=32, \delta=0.89$ e $\delta=1.30$. Os zeros nesse caso são obtidos nos pontos de cruzamento das linhas vermelhas com as pretas. Nesta dissertação estamos interessados na obtenção do zero mais próximo ao eixo real. O estágio posterior desse processo consiste no refinamento desses zeros com o algoritmo de minimização Amoeba. Na figura 6.6 ilustramos os zeros da função de partição em $u$ de uma série produzida para $L=32$, para $\delta=0.89$ e 1.30 , com ampliação na região de interesse. A partir da análise de escala de tamanho finito para a parte imaginária do primeiro zero complexo,

$$
\operatorname{Im}\left[u_{1}^{0}(L)\right] \propto L^{\frac{-1}{\nu}}
$$

obtemos o valor de $\nu$ e consequentemente a natureza da transição. Uma transição de primeira ordem ocorre quando $d \nu=1$. Também obtemos a temperatura crítica a partir da relação de escala

$$
\operatorname{Re}[\beta(L)]=\beta_{c}+a L^{\frac{-1}{\nu}}
$$

Com $\operatorname{Re}[\beta(L)]$ e $\nu$, é possível obter $\beta_{c}$ do gráfico $\operatorname{Re}[\beta(L)]$ versus $L^{\frac{-1}{\nu}}$. 


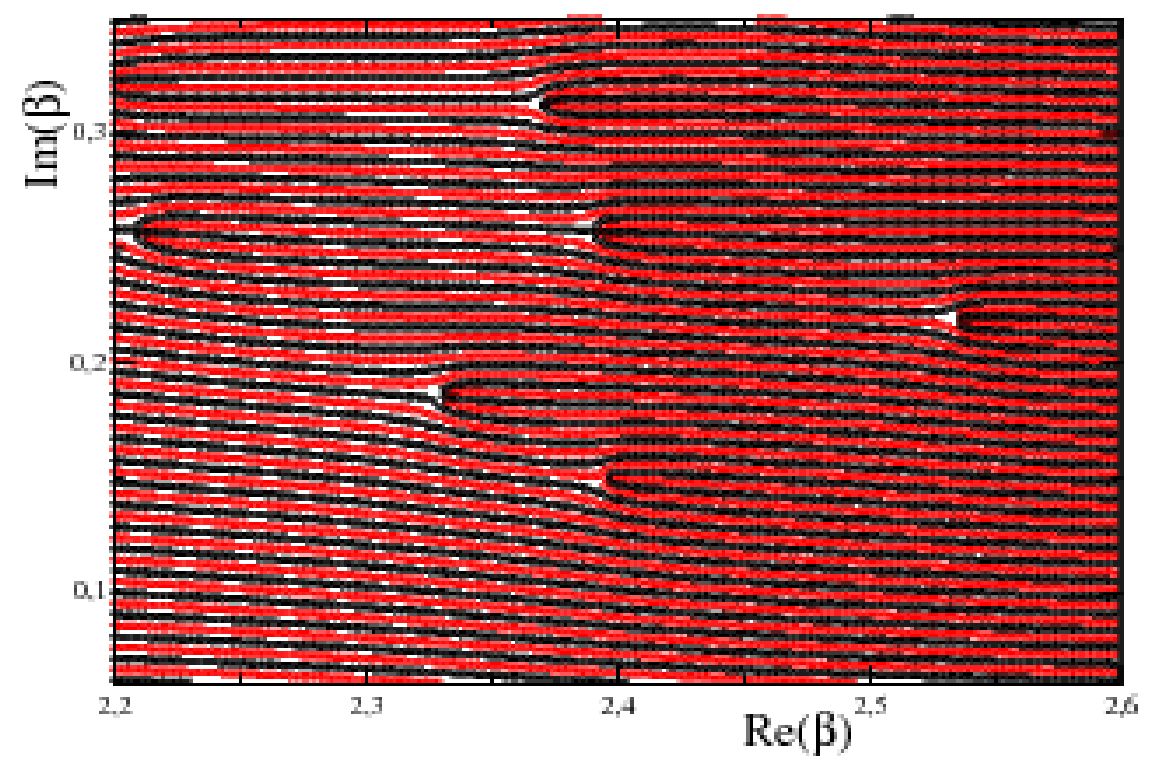

(a) $\delta=0.89$

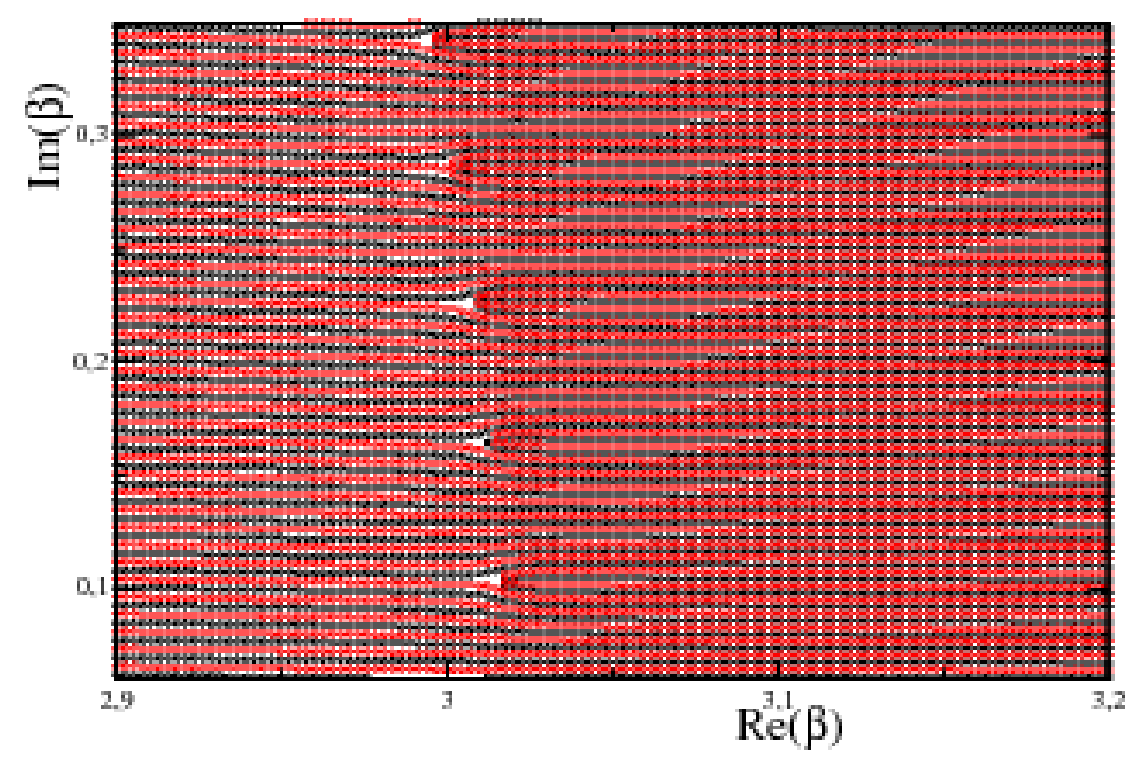

(b) $\delta=1.30$

Figura 6.5: Escaneamento dos zeros da função de partição canônica em $\beta$ para $L=32$. 


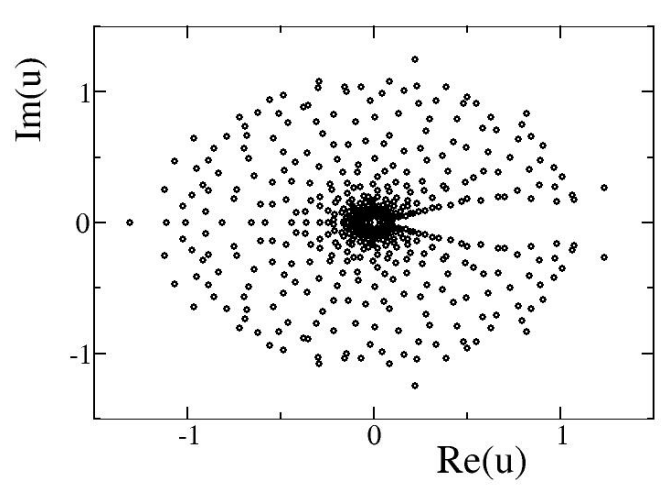

(a)

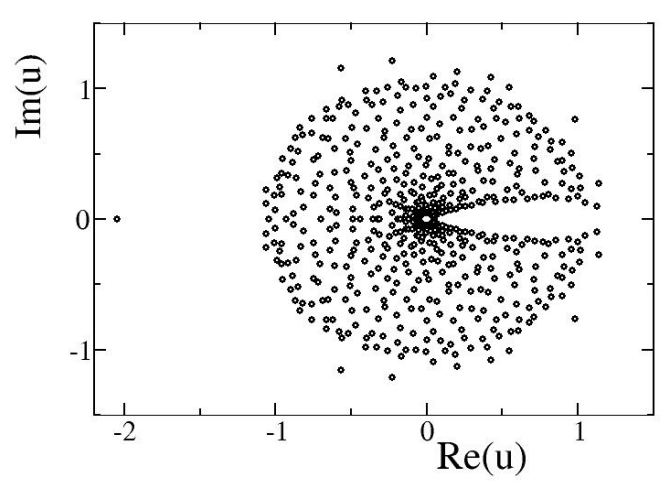

(c)

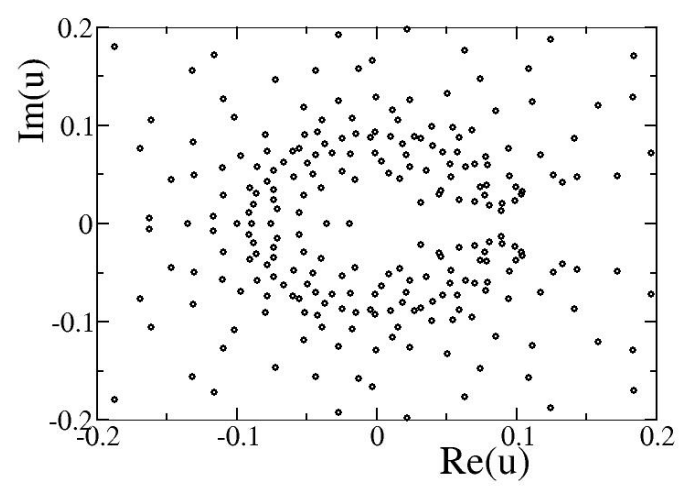

(b)

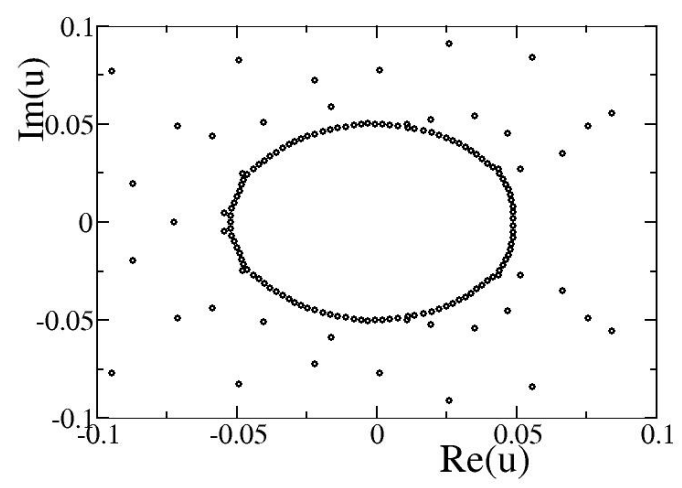

(d)

Figura 6.6: Zeros em $u=e^{-\beta}$ para $L=32$ obtidos de uma das 16 séries produzidas para (a) $(\delta=0.89)$ todos os zeros obtidos, (b) $(\delta=0.89)$ ampliação da região de interesse, $(\mathrm{c})(\delta=1.30)$ todos os zeros obtidos, $(\mathrm{d})(\delta=1.30)$ ampliação da região de interesse. 


\section{CApÍtulo}

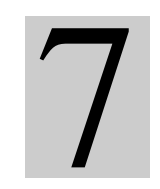

\section{RESUlTAdos E DISCUSSÃO}

Discutiremos neste capítulo os resultados obtidos para diversos valores de $\delta$ na região de interesse do diagrama de fase em função dos tamanhos de rede $L$ que utilizamos. O estudo do modelo estava inicialmente focado na região do diagrama de fase no intervalo $\delta \in[0.89,1.00]$. Tal escolha se deve ao fato, como já visto no capítulo 4, de haver evidências na literatura da existência de um ponto tricrítico nessa região. Entretanto, neste trabalho, o ponto tricrítico não foi localizado nessa região do diagrama de fase mencionada cima. Foi necessário então estender o estudo para $\delta>1.0$ à procura desse ponto. O estudo acabou abrangendo o intervalo $\delta \in[0.89,1.30]$. Com os resultados obtidos foi possível construir o diagrama de fase $T \times \delta$ para o intervalo de $\delta$ estudado e assim, localizar a região em que se encontra o ponto tricrítico. Esse ponto corresponde na literatura, à mudança da ordem de transição da fase do tipo faixas para o líquido tetragonal. Entretanto, encontramos indícios, a partir de uma análise microcanônica, de que a esta linha de transição de fase pode se dividir em duas outras linhas correspondentes às transições da fase do tipo faixas para a fase nemática e da fase nemática para o líquido tetragonal. Assim, o ponto tricrítico, se ocorrer, pode estar localizado na linha de transição da fase nemática para a tetragonal. A partir da análise de FSS para os zeros da função de partição, obtivemos como resultado uma única linha de transição do diagrama de fase, sendo que, para todos os valores do acoplamento $\delta$ abaixo de 1.20, essa linha representa uma transição de segunda ordem e para $\delta=1.30$, uma transição de primeira ordem. 


\begin{tabular}{ccc}
\hline$\delta$ & $d \nu$ & ordem da transição \\
\hline 0.89 & $1.79(8)$ & segunda \\
0.91 & $1.82(7)$ & segunda \\
0.93 & $1.77(7)$ & segunda \\
0.95 & $1.73(6)$ & segunda \\
0.97 & $1.71(6)$ & segunda \\
1.00 & $1.65(5)$ & segunda \\
1.10 & $1.42(3)$ & segunda \\
1.20 & $1.23(2)$ & segunda \\
1.30 & $1.019(5)$ & primeira \\
\hline
\end{tabular}

Tabela 7.1: Resultados obtidos a partir da FSS para os zeros da função de partição.

\subsection{Análise de escala de tamanho finito para os zeros da função de partição}

Vamos apresentar nesta seção os resultados obtidos pela análise de tamanho finito para os zeros da função de partição canônica. Todo o processo de produção de dados e de análise já foi descrito no capítulo 6 . O primeiro zero complexo nas variáveis $u$ e $\beta$ foi obtido a partir da média entre as 16 séries produzidas. A lista completa dos zeros, incluindo os respectivos erros estatisticos, está exposta em forma de tabela, para cada valor de $L$ e $\delta$ no apêndice B. As figuras 7.1 e 7.2 foram construídas a partir da parte imaginária dos zeros na variável $u$. Temos nessas figuras os gráficos de $-\ln \operatorname{Im}\left[u_{1}^{0}(L)\right]$ versus $\ln L$ com as respectivas barras de erro e os valores encontrados para $d \nu$ a partir da regressão linear. Vale lembrar que quando $d=1 / \nu$, temos uma transição de primeira ordem. Isso ocorre, dentre os valores de $\delta$ estudados, somente para $\delta=1.30$. Para todos os outros valores de $\delta$, nossos resultados apontaram para uma transição de segunda ordem. A tabela 7.1 apresenta o resumo desses resultados.

Nas figuras 7.3 e 7.4 temos os gráficos de $\operatorname{Re}[\beta(L)]$ versus $L^{-1 / \nu}$ com os respectivos valores de $\beta_{c}$ e $T_{c}$. Resumimos estes resultados na tabela 7.2. Com nossos valores de $T_{c}$, construimos a diagrama de fase apresentado na figura $7.5 \mathrm{com}$ o objetivo de compararmos com o intervalo correspondente do diagrama apresentado 


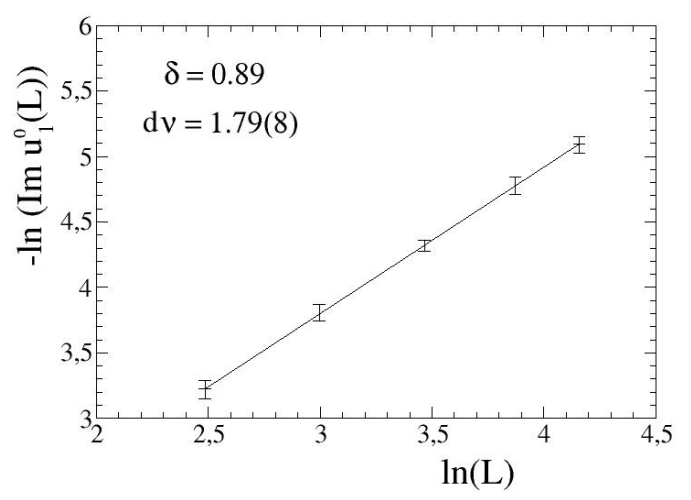

(a) $\delta=0.89$

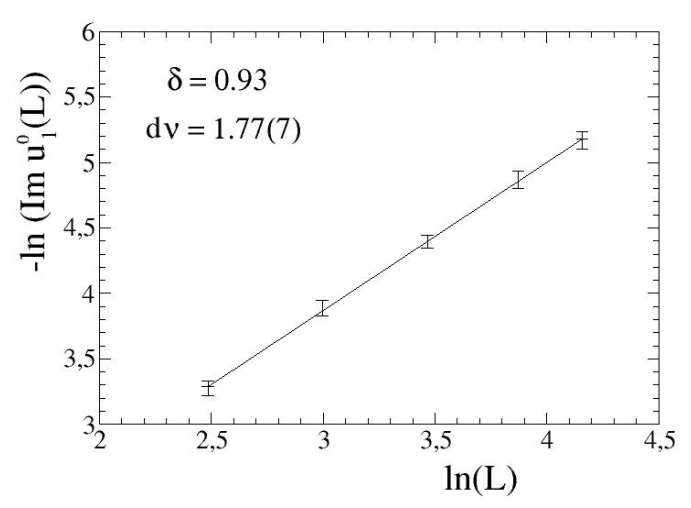

(c) $\delta=0.93$

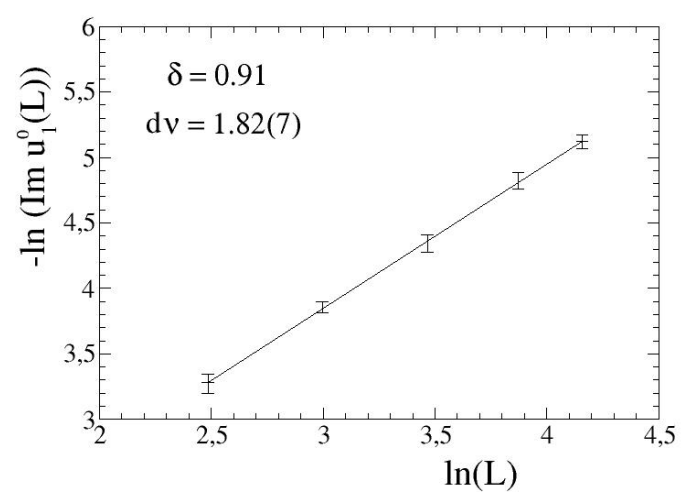

(b) $\delta=0.91$

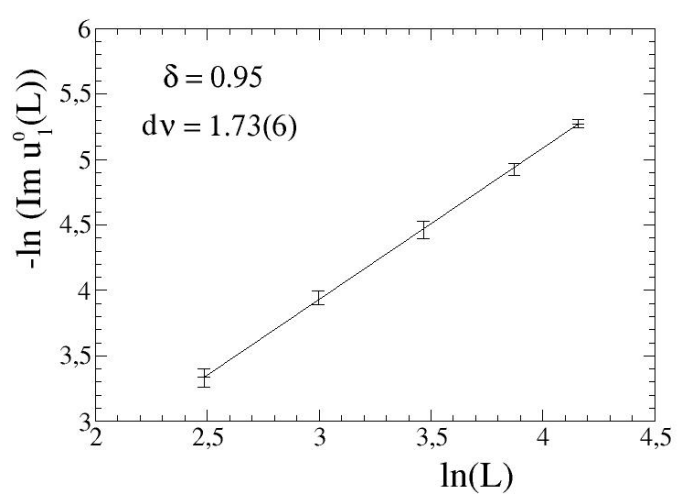

(d) $\delta=0.95$

Figura 7.1: Regressão linear para $-\ln \operatorname{Im}\left[u_{1}^{0}(L)\right]$ versus $\ln L$ com os respectivos valores de $d \nu$ para diferentes valores de $\delta$.

em (11). O diagrama de fase da ref. (11), já mostrado no capítulo 4, foi novamente inserido aqui (figura 7.6) para facilitar ao leitor a comparação entre os resultados. Observamos somente uma linha de transição no plano complexo da temperatura, correspondente à transição da fase do tipo faixas para o líquido tetragonal, em concordância com os resultados de simulações de Monte Carlo apresentados em (11). Diferentemente do que esperávamos observar, o diagrama 7.5 apresenta uma transição de primeira ordem somente em $\delta=1.30$, quando o estado fundamental é o estado do tipo faixas com $h=2$. Os nossos resultados a partir do estudo dos zeros complexos da função de partição indicam que a transição da fase do tipo faixas $h=1$ para a fase tetragonal é sempre contínua no intervalo $\delta \in[0.89,1.20]$. Aumentando 


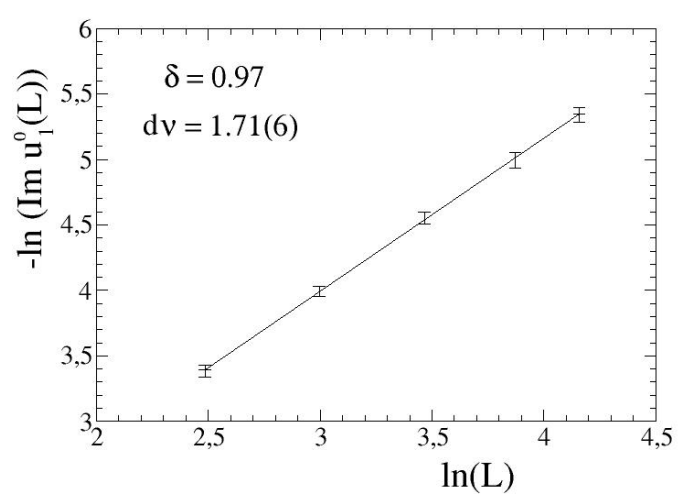

(a) $\delta=0.97$

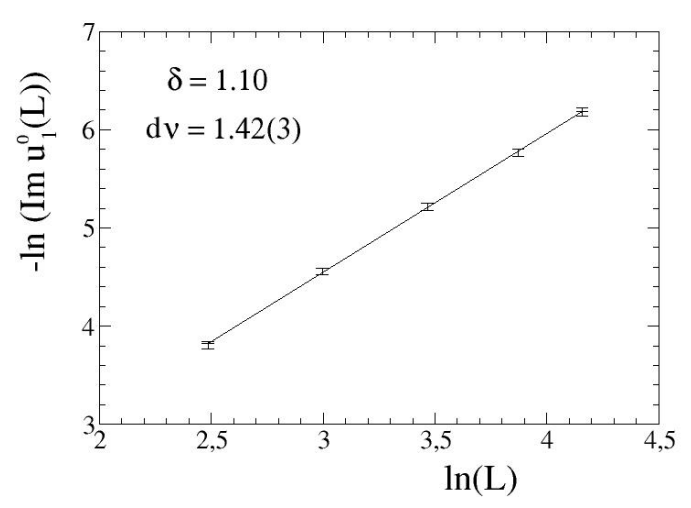

(c) $\delta=1.10$

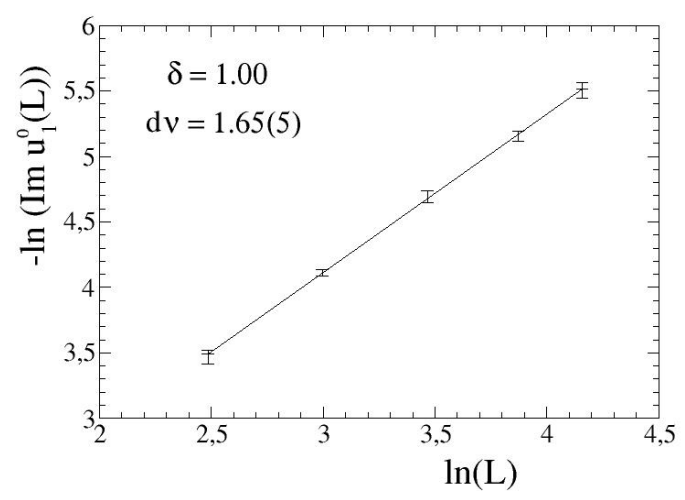

(b) $\delta=1.00$

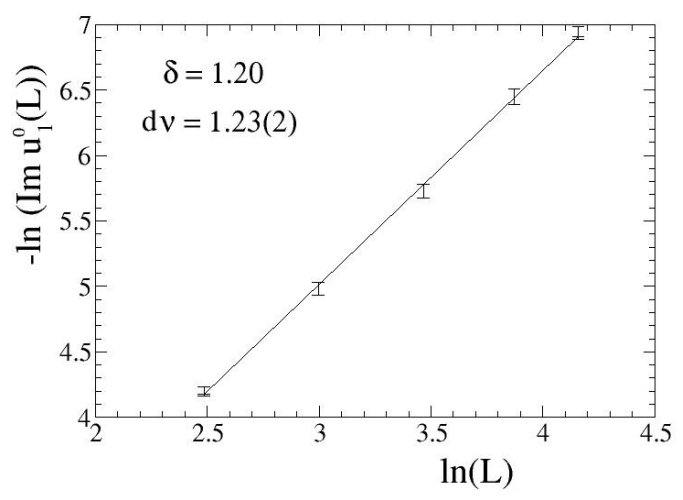

(d) $\delta=1.20$

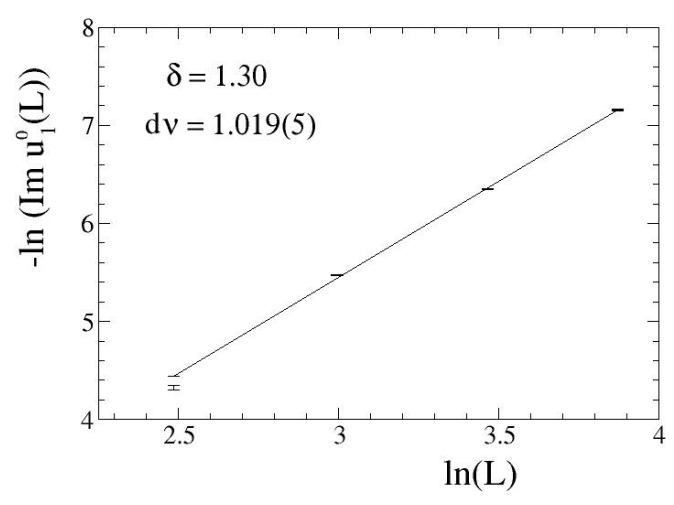

(e) $\delta=1.30$

Figura 7.2: Regressão linear para $-\ln \operatorname{Im}\left[u_{1}^{0}(L)\right]$ versus $\ln L$ com os respectivos valores de $d \nu$ para diferentes valores de $\delta$. 
o valor de $\delta$ para 1.30, a transição da fase do tipo faixas, com $h=2$, para a fase tetragonal passa a ser de primeira ordem. Estamos produzindo novas simulações para o intervalo $\delta \in[1.2,1.3]$ para obter com maior precisão o ponto tricrítico. Podemos pensar na possibilidade deste ponto ocorrer juntamente com a transição de $h=1$ para $h=2$. Vale lembrar que na ref. (10), os autores trabalharam com $L=48$ na obtenção dos resultados para $\delta=1.00$. Apesar de apontarem a existência de uma transição de primeira ordem fraca, deixaram claro a necessidade de novos estudos para confirmar a ordem da transição para esse acoplamento. Lembramos que o diagrama 7.6 contém informações coletadas de diversas referências. A transição de segunda ordem em $\delta=0.85$, por exemplo, é obtida da ref. (12).

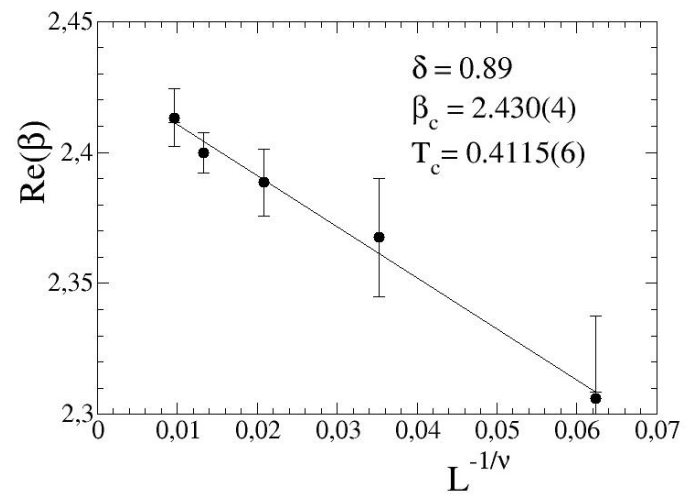

(a) $\delta=0.89$

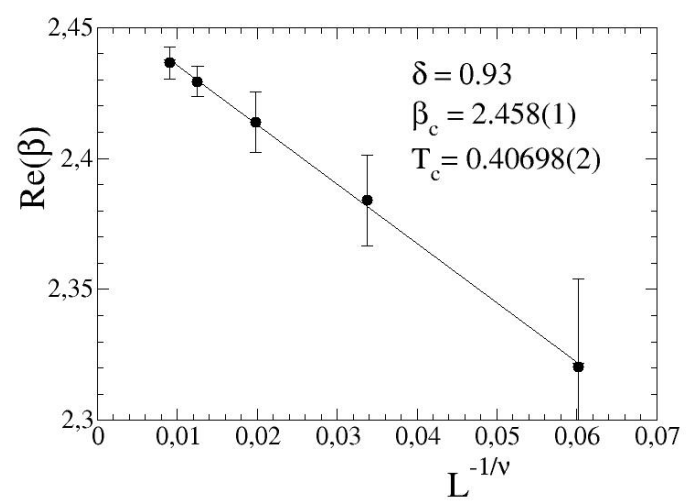

(c) $\delta=0.93$

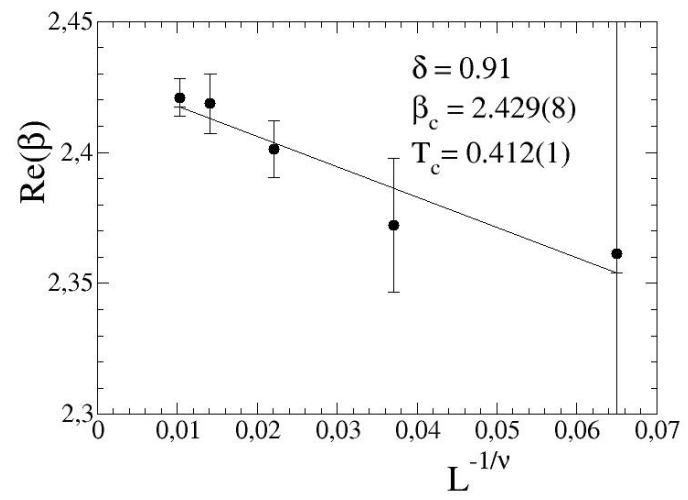

(b) $\delta=0.91$

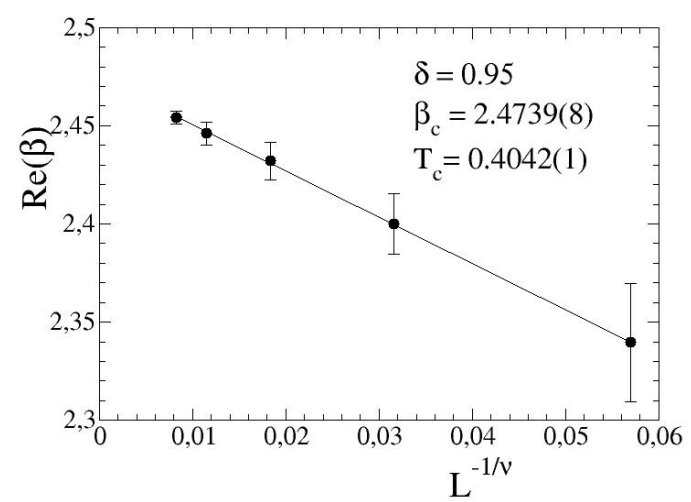

(d) $\delta=0.95$

Figura 7.3: Regressão linear para $\operatorname{Re} \beta(L)$ versus $L^{-1 / \nu}$ com os respectivos valores de $\beta_{c}$ e $T_{c}$. 


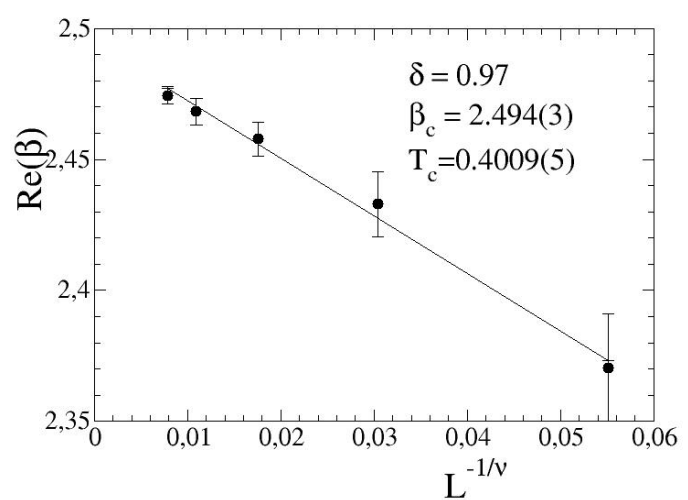

(a) $\delta=0.97$

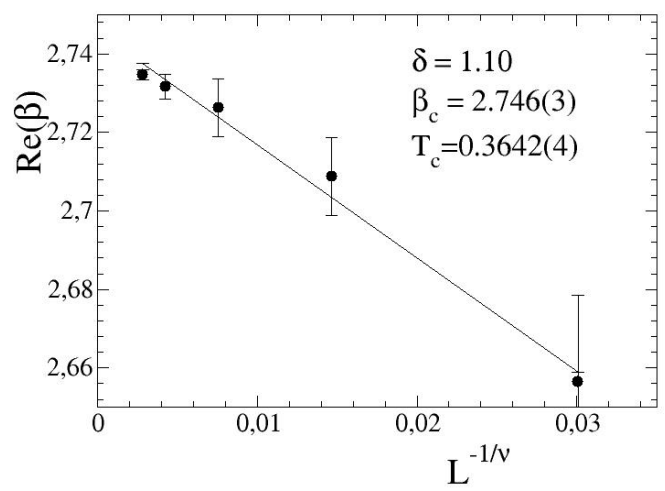

(c) $\delta=1.10$

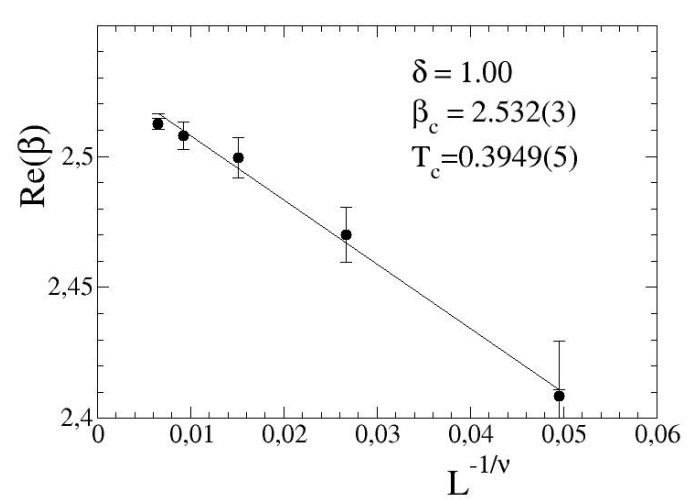

(b) $\delta=1.00$

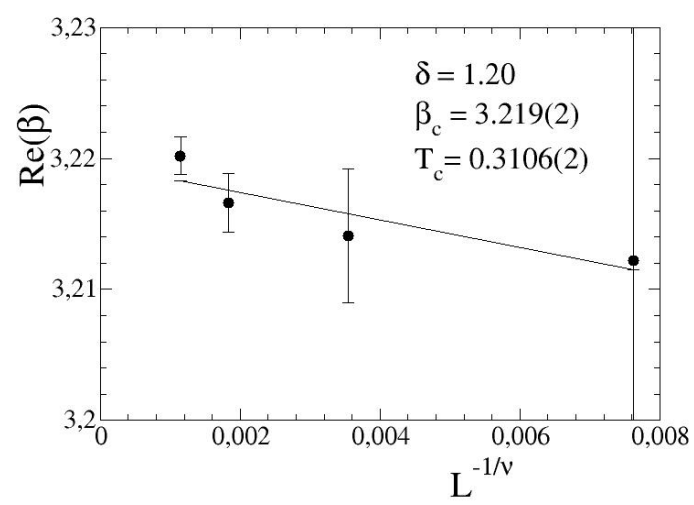

(d) $\delta=1.20$

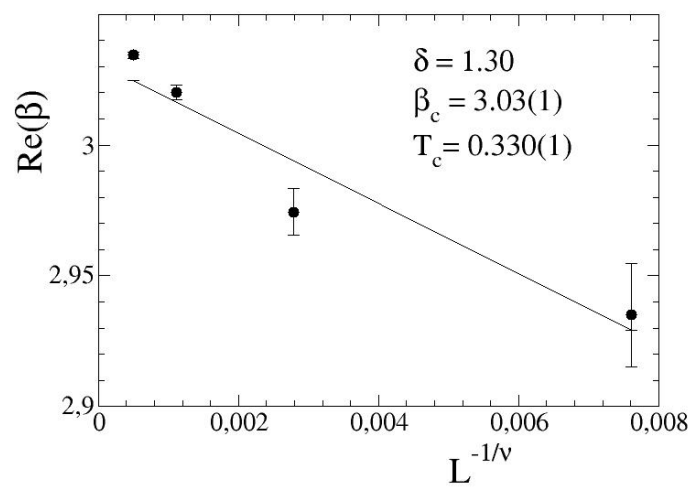

(e) $\delta=1.30$

Figura 7.4: Regressão linear para $\operatorname{Re} \beta(L)$ versus $L^{-1 / \nu}$ com os respectivos valores de $\beta_{c}$ e $T_{c}$. 


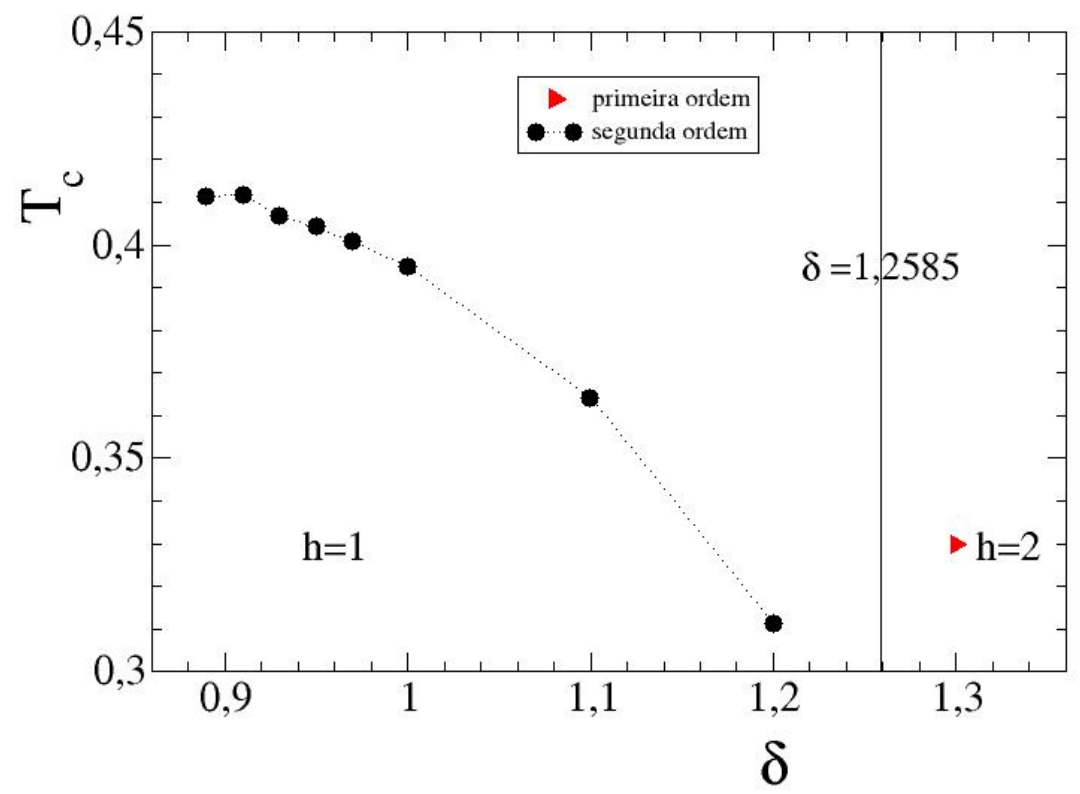

Figura 7.5: Diagrama de fase de $T_{c}$ versus $\delta$ construído a partir de nossos resultados.

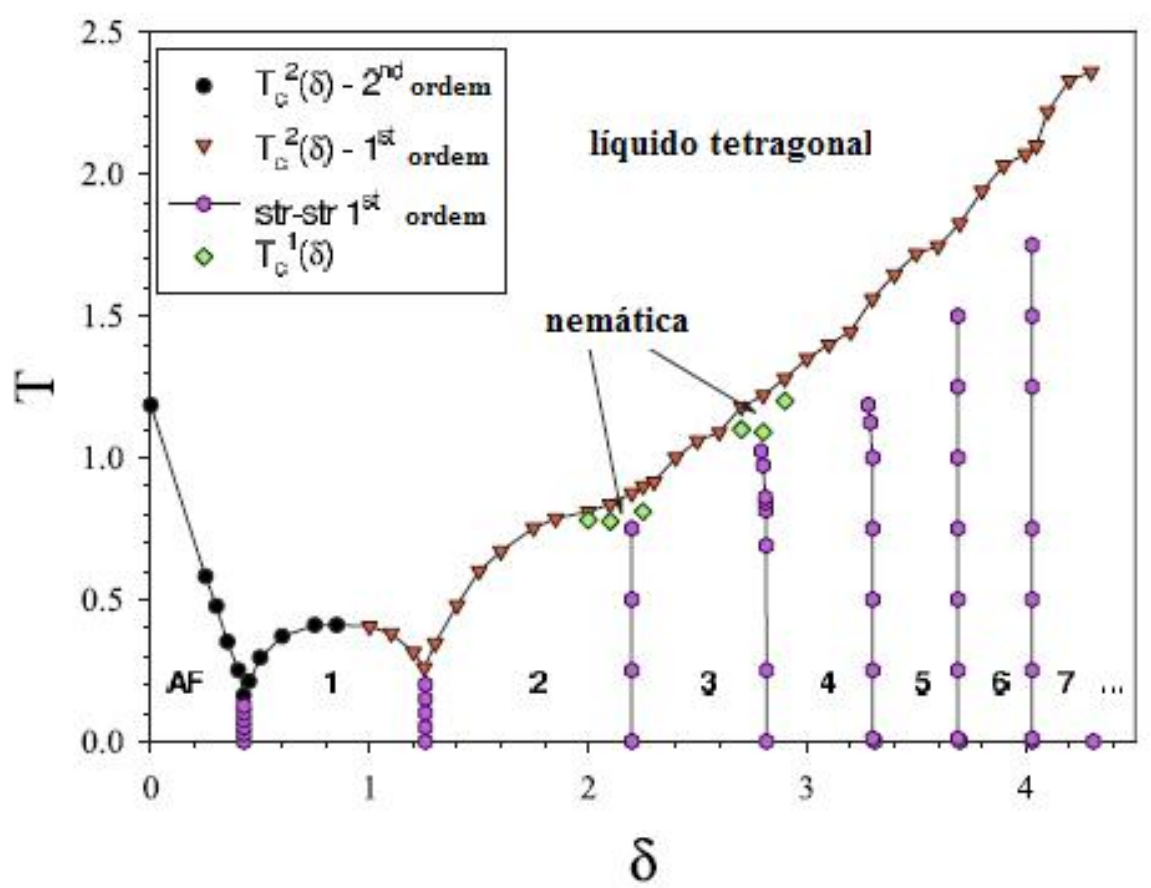

Figura 7.6: Diagrama de fase de $T_{c}$ versus $\delta$ retirado da ref.(11). 
7.1 - Análise de escala de tamanho finito para os zeros da função de partição72

\begin{tabular}{ccc}
\hline$\delta$ & $\beta_{c}$ & $T_{c}$ \\
\hline 0.89 & $2.430(4)$ & $0.4115(6)$ \\
0.91 & $2.429(8)$ & $0.412(1)$ \\
0.93 & $2.458(1)$ & $0.4068(2)$ \\
0.95 & $2.4739(8)$ & $0.4042(1)$ \\
0.97 & $2.494(3)$ & $0.4009(5)$ \\
1.00 & $2.532(3)$ & $0.3949(5)$ \\
1.10 & $2.746(3)$ & $0.3642(4)$ \\
1.20 & $3.219(2)$ & $0.3112(4)$ \\
1.30 & $3.03(1)$ & $0.330(1)$ \\
\hline
\end{tabular}

Tabela 7.2: Temperaturas críticas obtidas a partir da FSS para $\operatorname{Re}(\beta)$.

Comparando os dois diagramas e olhando para a tabela 7.2, observamos que os valores e comportamento das temperaturas críticas em função de $\delta$ são bem semelhantes: $T_{c}$ está em torno de 0.4 para $\delta=0.9$, e diminui progressivamente até a temperatura de transição entre as fases $h=1$ e $h=2$ e depois começa a aumentar. Note que $T_{c}$ em $\delta=1.30$ é ligeiramente maior que em $\delta=1.20$ nos dois diagramas. 


\subsection{Possibilidade de outra transição de fase}

Apesar dos nossos resultados, obtidos a partir das relacoes de FSS para os zeros complexos, indicarem somente uma linha de transição de fase na região de $\delta$ estudada e nos levar a relacionar essa linha com a transição da fase do tipo faixas para a tetragonal, os resultados obtidos com a análise microcanônica, descrita no apêndice $\mathrm{C}$, mostra a existência de duas transições de fase para $\delta=1.20$ e $\delta=1.30$. Os calores específicos observados para $\delta=1.20$ são característicos de transições de segunda ordem, enquanto que, para $\delta=1.30$, são característicos de primeira ordem (valores negativos). Essas duas transições provavelmente não foram observadas pela análise dos zeros complexos na variável temperatura porque as duas temperaturas críticas estão muito muito próximas e assim, não conseguimos identificar duas possíveis curvas de zeros complexos. A proximidade entre essas temperaturas foi verificada da seguinte forma: a partir dos gráficos de $C_{v}$ versus $E / N$ (vide figuras C.3b e C.4b), identificamos os valores de de $E / N$ para os quais ocorrem o pico no calor específico (no caso de $C_{v}<0$, escolhemos o valor intermediário entre o início e o final da ocorrência de valores negativos). Com os valores de $E / N$ em mãos, recorremos agora ao ajuste feito no gráfico de $b(E)$ (vide figura C.3a e C.4a) e localizamos o valor de $b_{c}(E)$ associado. Na tabela 7.3 colocamos os valores aproximados de $b_{c}(E)$, obtidos pela a análise microcanônica, juntamente com os valores de $\beta_{c}$ obtidos pela análise de FSS para os zeros da função de partição canônica. Note que o erro em $\beta$ da análise canônica é da mesma ordem que o algarismo que diferencia os valores $b_{c}(E)$ referentes à transições de fase diferentes. Fica claro que a diferença entre as duas linhas de transição no plano complexo da temperatura é tão tênue em comparação à precisão de nossas amostragens, que não pode ser observada. Faz-se necessário agora uma melhor investigação da região do diagrama de fase $\delta \in[1.20,1.30]$. Para tanto, estamos trabalhando na produção de dados para redes maiores com o intuito de observar duas transições de fase no plano complexo da temperatura e de se conseguir uma análise microcanônica mais apurada. 


\begin{tabular}{ccc}
\hline \multicolumn{3}{c}{$\delta=1.20$} \\
\hline$E / N$ & $b_{c}$ & $\beta_{c}(E)$ \\
\hline-0.432 & 3.224 & $3.219(2)$ \\
-0.416 & 3.221 \\
\hline \hline \multicolumn{3}{c}{$\delta=1.30$} \\
\hline$E / N$ & $b_{c}$ & $\beta_{c}(E)$ \\
\hline-0.474 & 3.01 & $3.03(1)$ \\
-0.440 & 3.05 & \\
\hline
\end{tabular}

Tabela 7.3: Comparação entre $b_{c}(E)$ e $\beta_{c}$ para $\delta=1.20$ e 1.30 . 


\section{Capítulo}

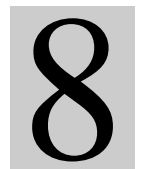

\section{CONCLUSÕES}

A partir dos resultados de FSS para os zeros da função de partição no plano complexo da temperatura, temos, no intervalo $\delta \in 0.89,1.20$, uma transição de segunda ordem, e para $\delta=1.30$, uma transição de primeira ordem. Para os tamanhos de redes utilizados observamos somente uma linha de transição de fase no plano complexo da temperatura. A análise microcanônica fornece indícios da presença de uma transição de fase de segunda ordem para $\delta \in[0.89,1.10]$, em concordância com a análise via zeros da função de partição. Entretanto, observamos duas transições de fase de segunda ordem para $\delta=1.20$ e duas transições de fase de primeira ordem para $\delta=1.30$. Assim, para esses dois valores de $\delta$ mencionados, não podemos identificar à qual transição de fase a linha de zeros observada no plano complexo da temperatura se relaciona, já que as temperaturas identificadas na análise microcanônica estão muito próximas uma da outra. No plano complexo, essas duas linhas de zeros estão tão próximas uma da outra que não conseguimos distinguí-las para os tamanho de redes utilizados nas simulações. Temos na literatura a presença da fase nemática entre as fases do tipo faixas com $h=2$ e $h=3$, entre $h=3$ e $h=4$, e assim por diante. Porém, a ocorrência da fase nemática entre $h=1$ e $h=2$ ainda não havia está relatada na literatura. O ponto tricrítico, objeto deste trabalho, não foi obtido com precisão, por causa da necessidade de explorarmos a região entre $\delta=1.1$ e $\delta=1.3$. Novas simulações, para redes maiores e também para novos valores de $\delta$ nessa região estão em andamento neste momento. Por enquanto, estamos produzindo dados para todos os valores de $\delta$ já estudados, mas com $L>64$ e também para os novos valores $\delta=1.15$ e 1.35 . Temos que o ponto procurado está 
localizado entre $\delta=1.20$ e 1.30 , diferentemente do que esperávamos no início do trabalho com base nas referências já citadas no decorrer da dissertação. O estudo de tal sistema para o intervalo de acoplamento $\delta$ estudado deve ser tratado com redes maiores para verificar a presença ou não da fase nemática nessa região do diagrama de fase e o descrevermos com mais detalhes. A partir das evidências encontradas neste trabalho, a região do diagrama de fase estudada se mostrou mais complexa do que esperávamos e o seu estudo não mais se resume à localização do ponto tricrítico. 


\section{REFERÊNCIAS BIBLIOGRÁFICAS}

1 ALLENSPACH, R.; BISCHOF, A. Magnetization direction switching in $\mathrm{Fe} / \mathrm{Cu}(100)$ epitaxial films: Temperature and thickness dependence. Physical Review Letters, v. 69, n. 23, p. 3385(6), dez. 1992.

2 AllensPACH, R.; STAMPANONI, M.; BISCHOF, A. Magnetic domains in thin epitaxial Co/Au(111) films. Physical Review Letters, v. 65, n. 26, p. 3344(6), dez. 1990.

3 MACiSAAC, A. B.; WhiteheAD, J. P.; DE'BELL, K. Monte Carlo study of two-dimensional Ising dipolar antiferromagnets as a model for rare-earth ordering in the $\mathrm{R}-\mathrm{Ba}-\mathrm{Cu}-\mathrm{O}$ compounds $(\mathrm{R}=$ rare earth $)$. Physical Review B, v. 46, n. 10, p. 6387(8), set. 1992.

4 MACISAAC, A. B.; WHITEHEAD, J. P. Striped phases in two-dimensional dipolar ferromagnets. Physical Review B, v. 51, n. 22, p. 16033(13), jun. 1995.

5 PORTMANN, O.; VATERLAUS, A.; PESCIA, D. An inverse transition of magnetic domain patterns in ultrathin films. Nature, v. 422, p. 701(4), abr. 2003.

6 TOUKMAJI, A. Y.; JR., J. A. B. Ewald summation techniques in perspective: a survey. Computer Physics Communications, v. 95, p. 73(19), jun. 1996.

7 WIDMANN, A. H.; ADOLF, D. B. A comparison of Ewald summation techniques for planar surfaces. Computer Physics Communications, v. 107, p. 167(20), dez. 1997.

8 RIZZI, L. G. Simulações Numéricas de Monte Carlo Aplicadas no Estudo das Transições de Fase do Modelo de Ising Dipolar Bidimensional. Dissertação (Mestrado) — Universidade de São Paulo, 2009. 
9 CAnnaS, S. A.; STARIOLO, D. A.; TAMARIT, F. A. Stripe-tetragonal first-order phase transition in ultrathin magnetic films. Physical Review B, v. 69, n. 9, p. 092409(4), mar. 2004.

10 CANNAS, S. A. et al. Ising nematic phase in ultrathin magnetic films: A Monte Carlo study. Physical Review B, v. 73, n. 18, p. 184425(12), maio 2006.

11 PIGHÍN, S. A.; CANNAS, S. A. Phase diagram of an Ising model for ultrathin magnetic films: Comparing mean field and Monte Carlo predictions. Physical Review B, v. 75, n. 22, p. 224433(9), jun. 2007.

12 RASTELli, E.; REGINA, S.; TASSI, A. Phase transitions in a square Ising model with exchange and dipole interactions. Physical Review B, v. 73, n. 14, p. 144418(11), abr. 2006.

13 RIZZI, L. G.; ALVES, N. A. Phase transitions and autocorrelation times in two-dimensional Ising model with dipole interactions. Physica B: Condensed Matter, v. 405, n. 6, p. 1571(9), mar. 2010.

14 PEARSON, R. B. Partition function of the Ising model on the periodic $4 \times 4 \times 4$ lattice. Physical Review B, v. 26, n. 11, p. 6285(6), dez. 1982.

15 BHANOT, G. et al. Accurate estimate of $\nu$ for the three-dimensional Ising model from a numerical measurement of its partition function. Physical Review Letters, v. 59, n. 7, p. 803(4), ago. 1987.

16 AlVES, N. A.; BERG, B. A.; VILlANOVA, R. Ising-model Monte Carlo simulations: Density of states and mass gap. Physical Review B, v. 41, n. 1, p. 383(12), jan. 1990.

17 Alves, N. A.; FEliCiO, J. R. D. de; HAnSMAnN, U. H. Partition function zeros and leading-order scaling correction of the $3 \mathrm{~d}$ Ising model from multicanonical simulations. Journal of Physics A: Mathematical and General, v. 33, p. 7489(9), jul. 2000 .

18 ITZYKSON, C.; PEARSON, R. B.; ZUBER, J. B. Distribuition of zeros in Ising and Gauge models. Nuclear Physics B, v. 220, p. 415(19), 1983. 
19 AlvES, N. A.; FELICIO, J. R. D. de; HANSMANN, U. H. A new look at the Ising model from exact partition functions zeros for large lattice sizes. International Journal of Modern Physics C, v. 8, n. 5, p. 1063(9), 1997.

20 AlveS, N. A.; BERG, B. A.; VILlAnOVA, R. Potts models: Density of states and mass gap from Monte Carlo simulations. Physical Review B, v. 43, n. 7, mar. 1991.

21 YANG, C. N.; LEE, T. D. Statistical theory of equations of state and phase transitions. i. theory of condensation. ii. lattice gas and Ising model. Physical Review, v. 87, n. 3, p. 404(16), ago. 1952.

22 BENA, I.; DROZ, M.; LIPOWSKI, A. Statistical mechanics of equilibrium and nonequilibrium phase transitions: The Yang-lee formalism. International Journal of Modern Physics B, v. 19, n. 29, p. 4269(61), 2005.

23 BERG, B. A.; NEUHAUS, T. Multicanonical algorithm for first order phase transitions. Physics Letters B, v. 267, p. 249(5), 1991.

24 BERG, B. A.; NEUHAUS, T. Multicanonical ensemble: A new approach to simulate first-order phase transitions. Physical Review Letters, v. 68, p. 9(4), 1992.

25 BERG, B. A.; HANSMANN, U. H.; NEUHAUS, T. Simulation of an ensemble with varying magnetic field: A numerical determination of the order-order interface tension in the $\mathrm{d}=2$ Ising model. Physical Review B, v. 47, p. 497(4), jan. 1993.

26 BERG, B. A. Multicanonical simulations step by step. Computer Physics Communications, v. 153, n. 3, p. 397(10), jul. 2003.

27 STANley, H. E. Introdution to Phase Transitions and Critical Phenomena. [S.l.]: Oxford University Press, 1971.

28 BORRMAnN, P.; MÜlkEn, O.; HARTING, J. Classification of phase transitions in small systems. Physical Review Letters, v. 84, n. 16, p. 3511(4), abr. 2000.

29 MARIS, H. J.; KADANOFF, L. P. Teaching the renormalization group. American Journal of Physics, v. 46, n. 6, p. 652(6), jun. 1978. 
30 ALVES, N. A.; HANSMANN, U. H. Partition function zeros and finite size scaling of helix-coil transitions in a polypeptide. Physical Review Letters, v. 84, n. 8 , p. $1836(4)$, fev. 2000.

31 RASTELLI, E.; REGINA, S.; TASSI, A. Phase transitions in a square Ising model with exchange and dipole interactions: Monte Carlo simulations. Physical Review B, v. 76, n. 5, p. 554438, ago. 2007.

32 BOOTH, I.; MACISAAC, A. B.; WHITEHEAD, J. P. Domain structures in ultrathin magnetic films. Physical Review Letters, v. 75, n. 5, p. 950(4), jul. 1995.

33 ABAnOV, A.; KAlATSKY, V.; POKROVSKY, V. L. Phase diagram of ultrathin ferromagnetic films with perpendicular anisotropy. Physical Review B, v. 51, n. 2, p. 1023(16), jan. 1995.

34 ARLETT, J. et al. Phase diagram for the striped phase in the two-dimensional dipolar Ising model. Physical Review B, v. 54, n. 5, p. 3394(9), ago. 1996.

35 BINDER, K. Introduction: theory an "technical" aspects of Monte Carlo simulations. In: Monte Carlo Methods in Statistical Physics. [S.l.]: Springer, 1979, (Topics in current physics, v. 7). p. 1(45).

36 Metropolis, N.; ROSEnBluth, A. W.; ROSEnBluth, M. N. Equation of state calculations by fast computing machines. Journal of Chemical Physics, v. 21, n. 6, jun. 1953 .

37 LEE, J. New Monte Carlo algorithm: Entropic sampling. Physical Review Letters, v. 71, n. 2, p. 211(4), jul. 1993.

38 WANG, F.; LANDAU, D. P. Efficient, multiple-range random walk algorithm to calculate the density of states. Physical Review Letters, v. 86, n. 10, p. 2050(4), mar. 2001.

39 HAO, M.-H.; SCHERAGA, H. A. Reply to comment on "Monte Carlo simulation of a first-order transition for protein folding". Journal of Physical Chemistry, v. 99, p. 2238(1), fev. 1995. 
40 BerG, B. A.; HANSMAnn, U. H.; OKAmotO, Y. Comment on "Monte Carlo simulation of a first-order transition for protein folding". Journal of Physical Chemistry, v. 99, p. 2236, fev. 1995.

41 BERG, B. A. Introdution to multicanonical Monte Carlo simulations. Fields Institute Communications, v. 26, p. 1, 2000.

42 PRESS, W. H. et al. Numerical recipes: the art of scientific computing. 2. ed. [S.1.]: Cambridge University Press, 1992. 


\section{APÊNDICE}

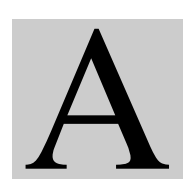

\section{PARÂMETROS MULTICANÔNICOS}

Apresentamos nas próximas páginas os parâmetros multicanônicos obtidos para todos os valores de $L$ em função de $\delta$. Estes parâmetros foram obtidos a partir da parte $\mathrm{B}$ descrita na seção 6.2 e foram utilizados para definir o peso multicanônico

$$
\omega_{m u}(E)=e^{-S}=e^{-b(E) E+a(E)}
$$

utilizado na produção de dados.

Vale lembrar que, na terminologia microcanônica, $S=-\ln \rho(E)$. Da relação termodinâmica, $F=E-T S, b(E)$ corresponde ao inverso da temperatura microcanônica $T(E)$, ou seja, $b(E)=\partial S / \partial E$, e $a(E)$ se associa com a energia livre de Helmholtz por $a(E)=F(E) / T(E)$. 

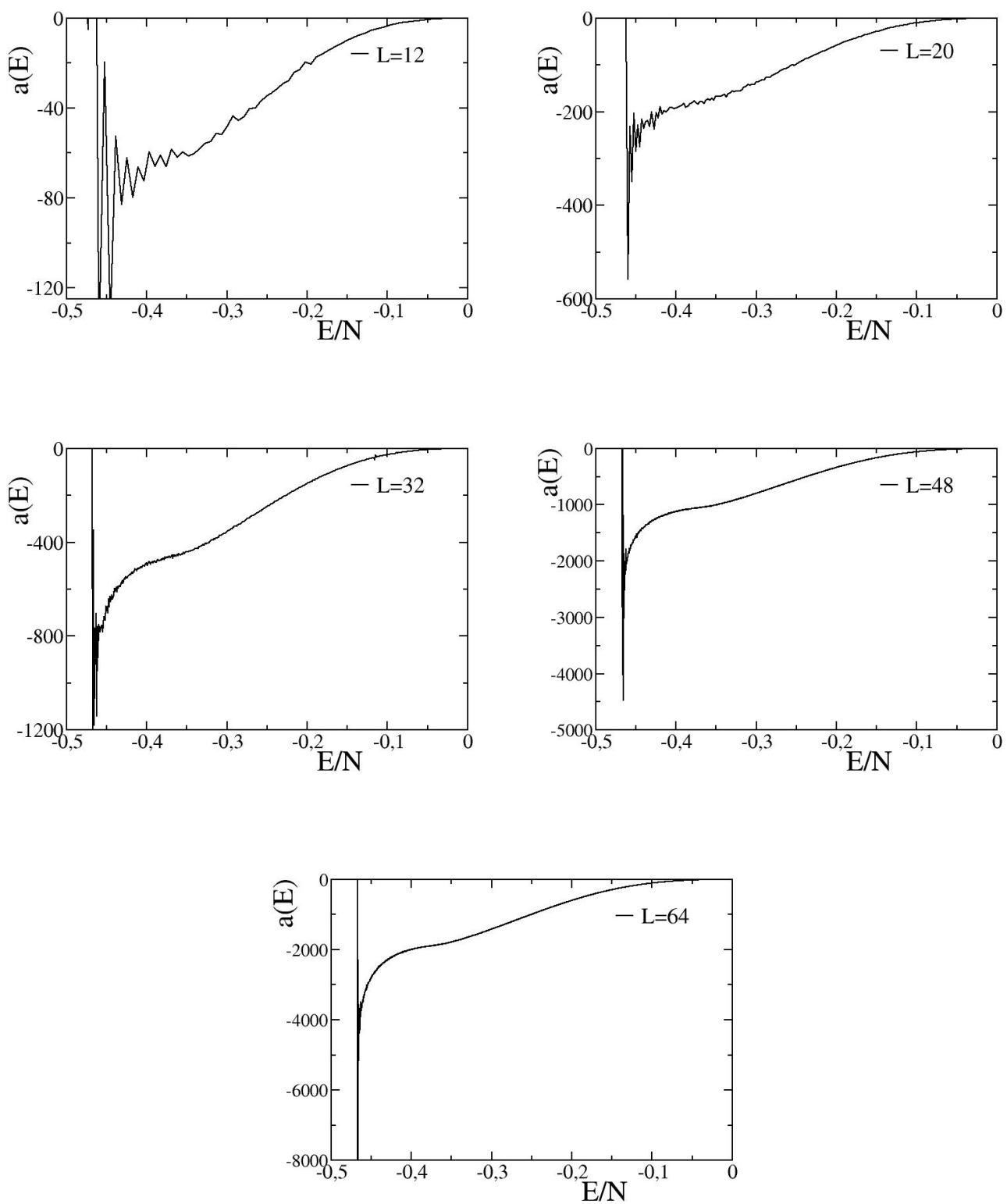

Figura A.1: Parâmetros multicanônicos $a(E)$ finais utilizados para a produção de dados para $\delta=0.89$. 

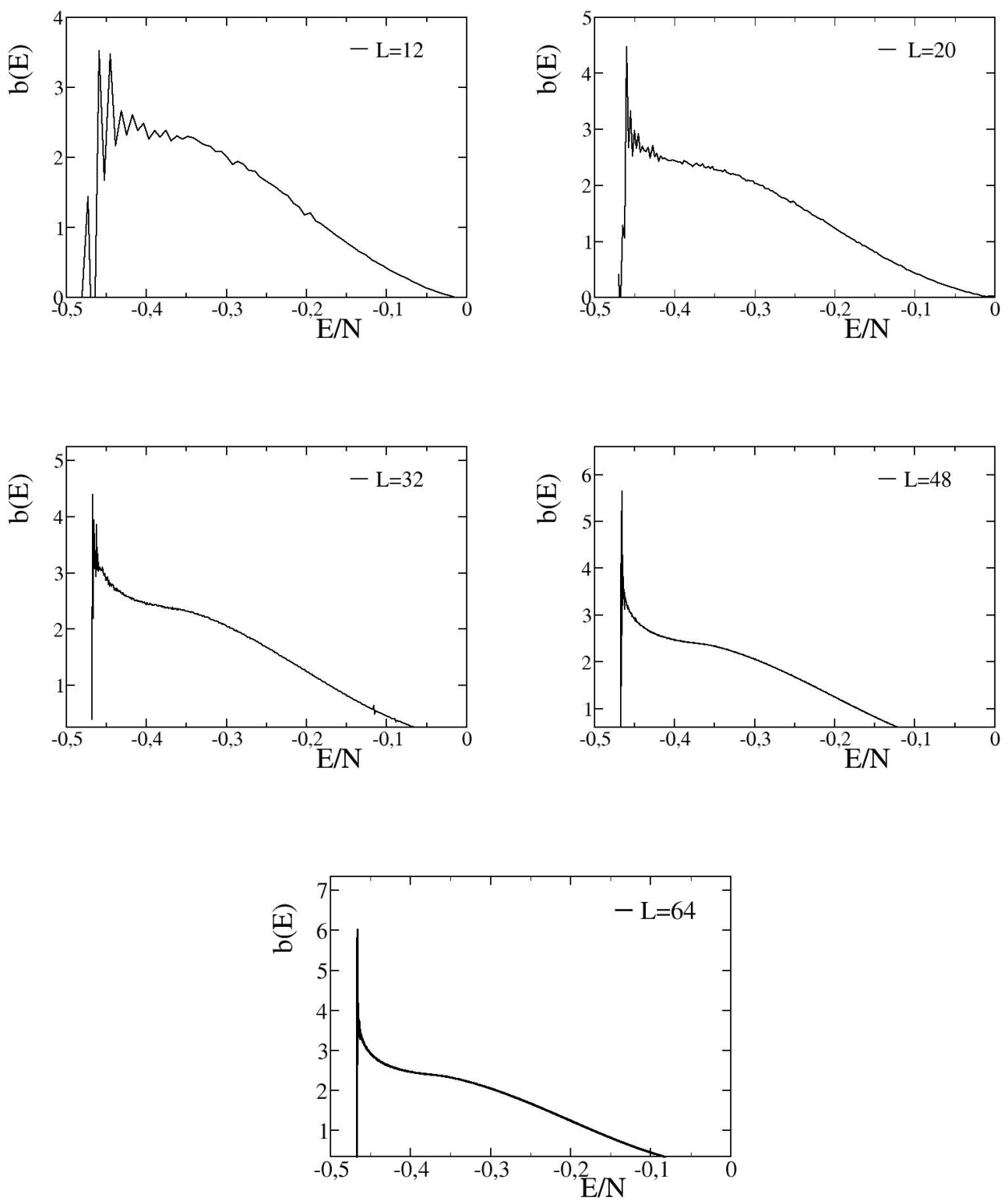

Figura A.2: Parâmetros multicanônicos $b(E)$ finais utilizados para a produção de dados para $\delta=0.89$. 

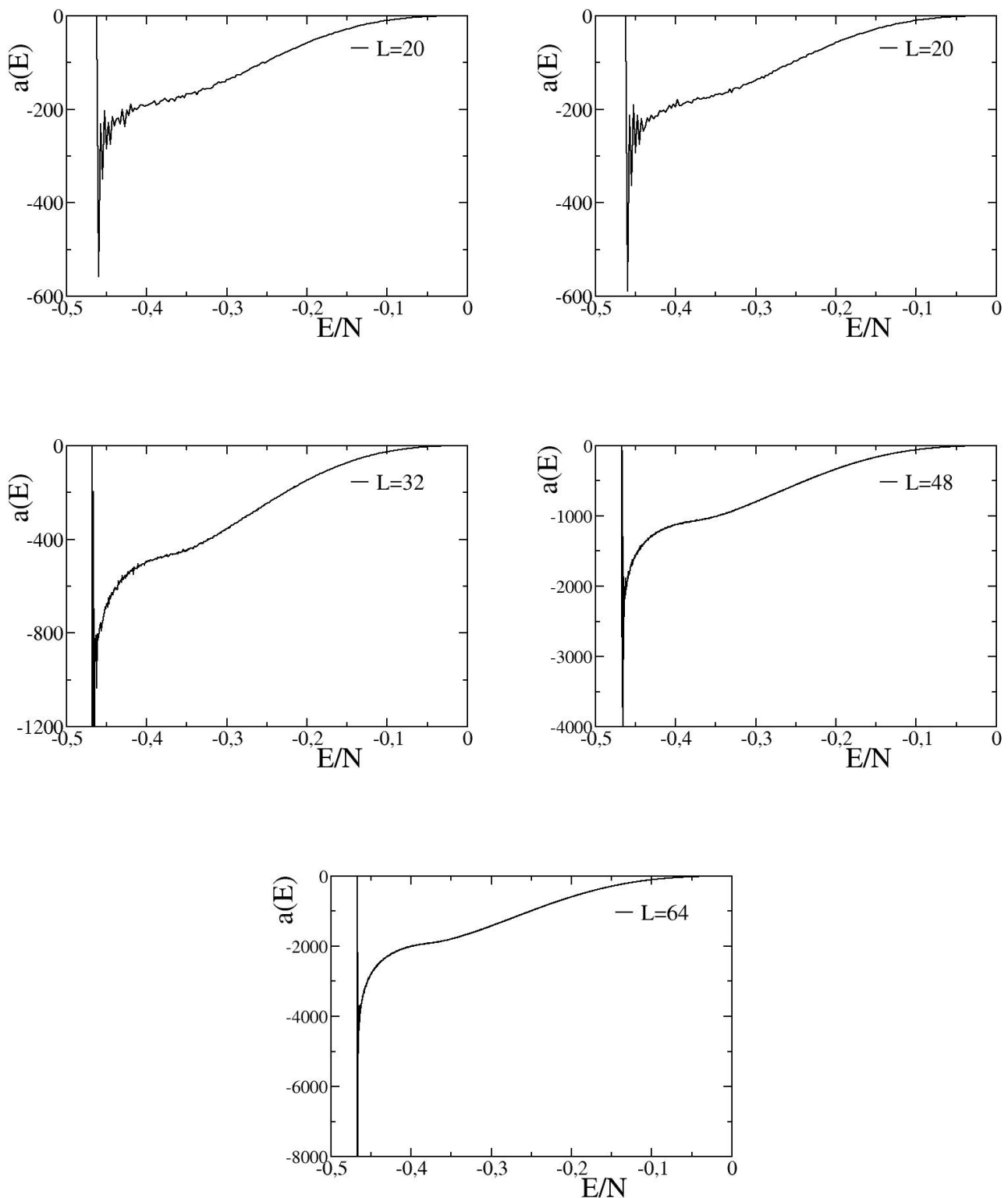

Figura A.3: Parâmetros multicanônicos $a(E)$ finais utilizados para a produção de dados para $\delta=0.91$. 

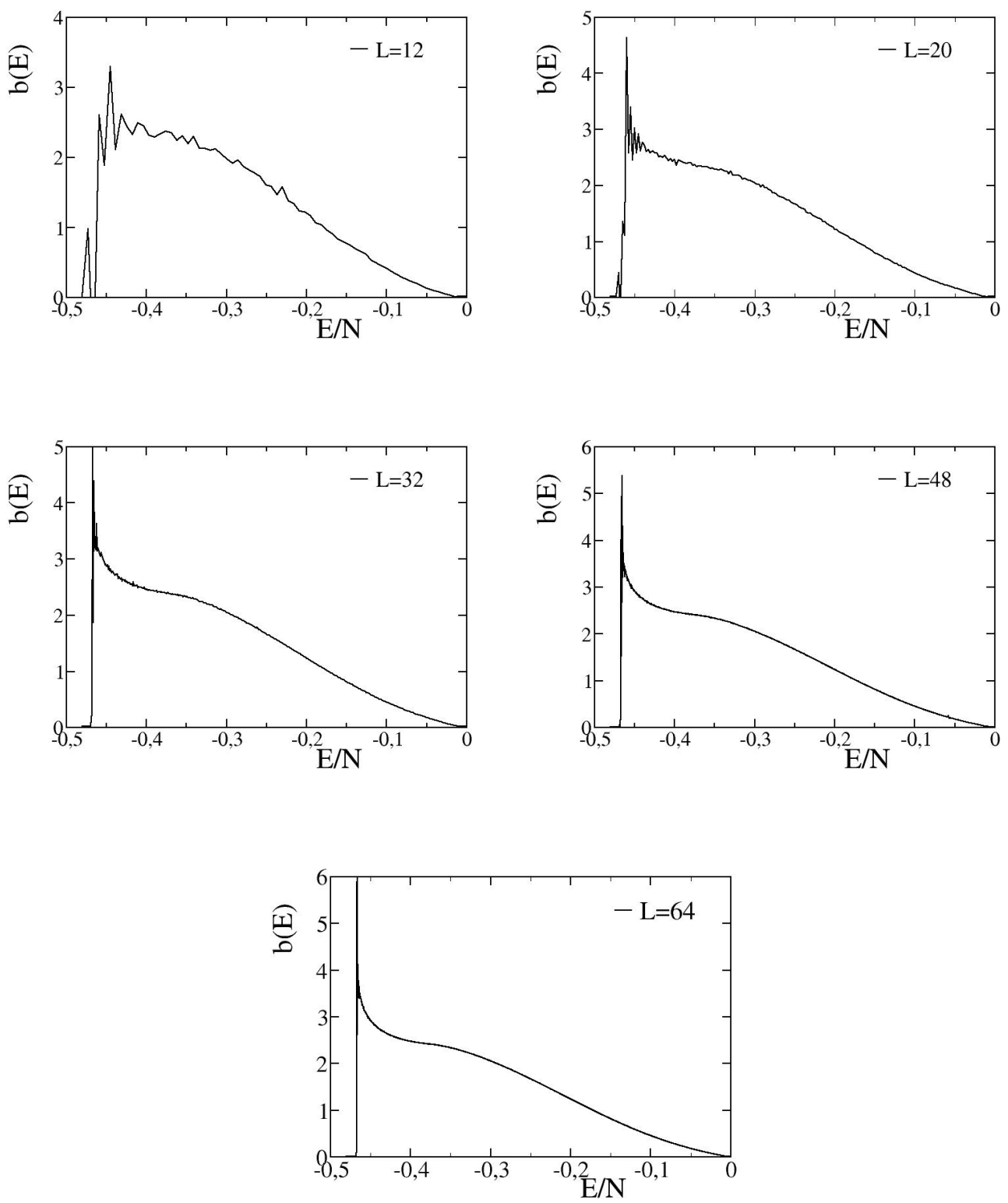

Figura A.4: Parâmetros multicanônicos $b(E)$ finais utilizados para a produção de dados para $\delta=0.91$. 

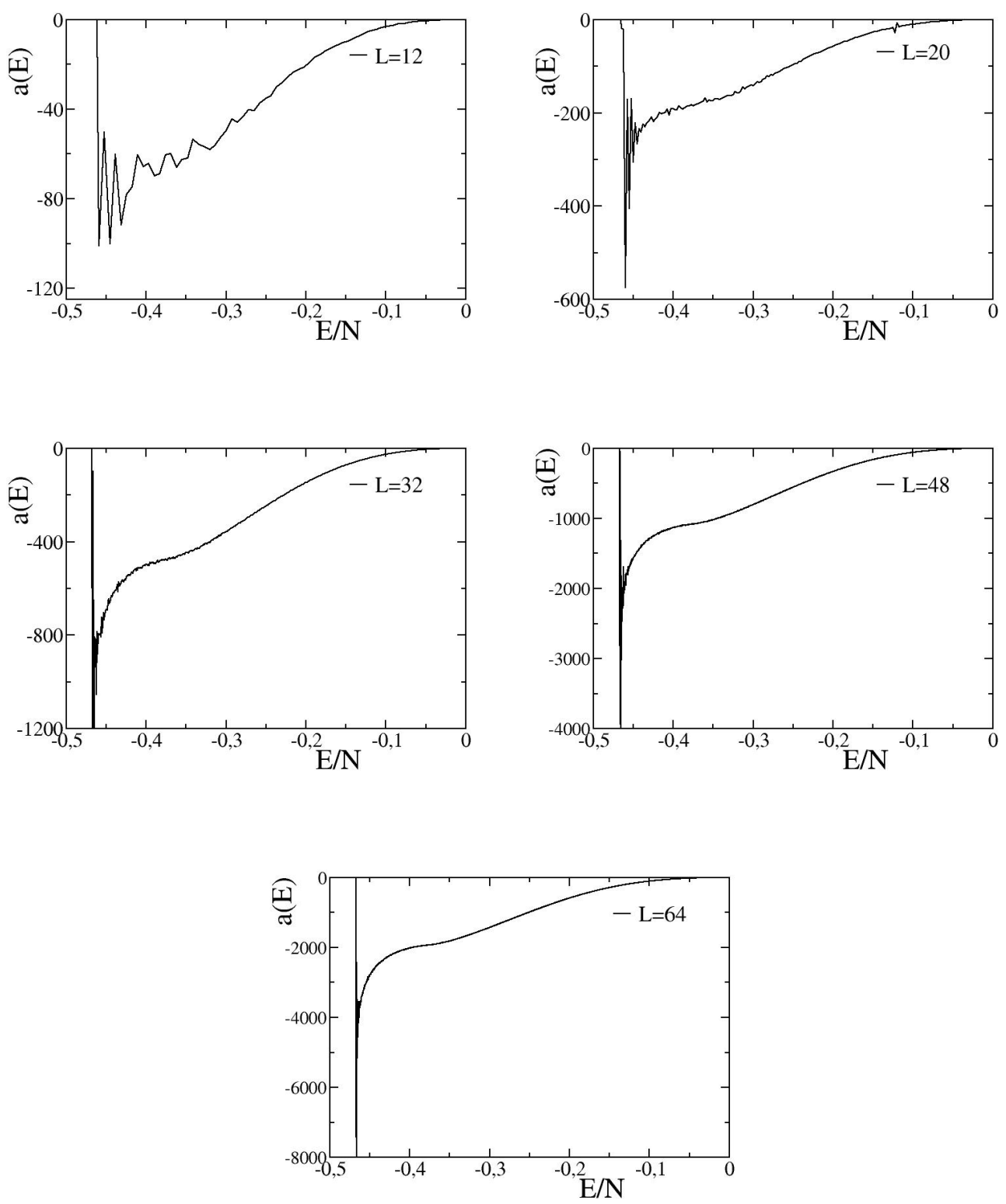

Figura A.5: Parâmetros multicanônicos $a(E)$ finais utilizados para a produção de dados para $\delta=0.93$. 

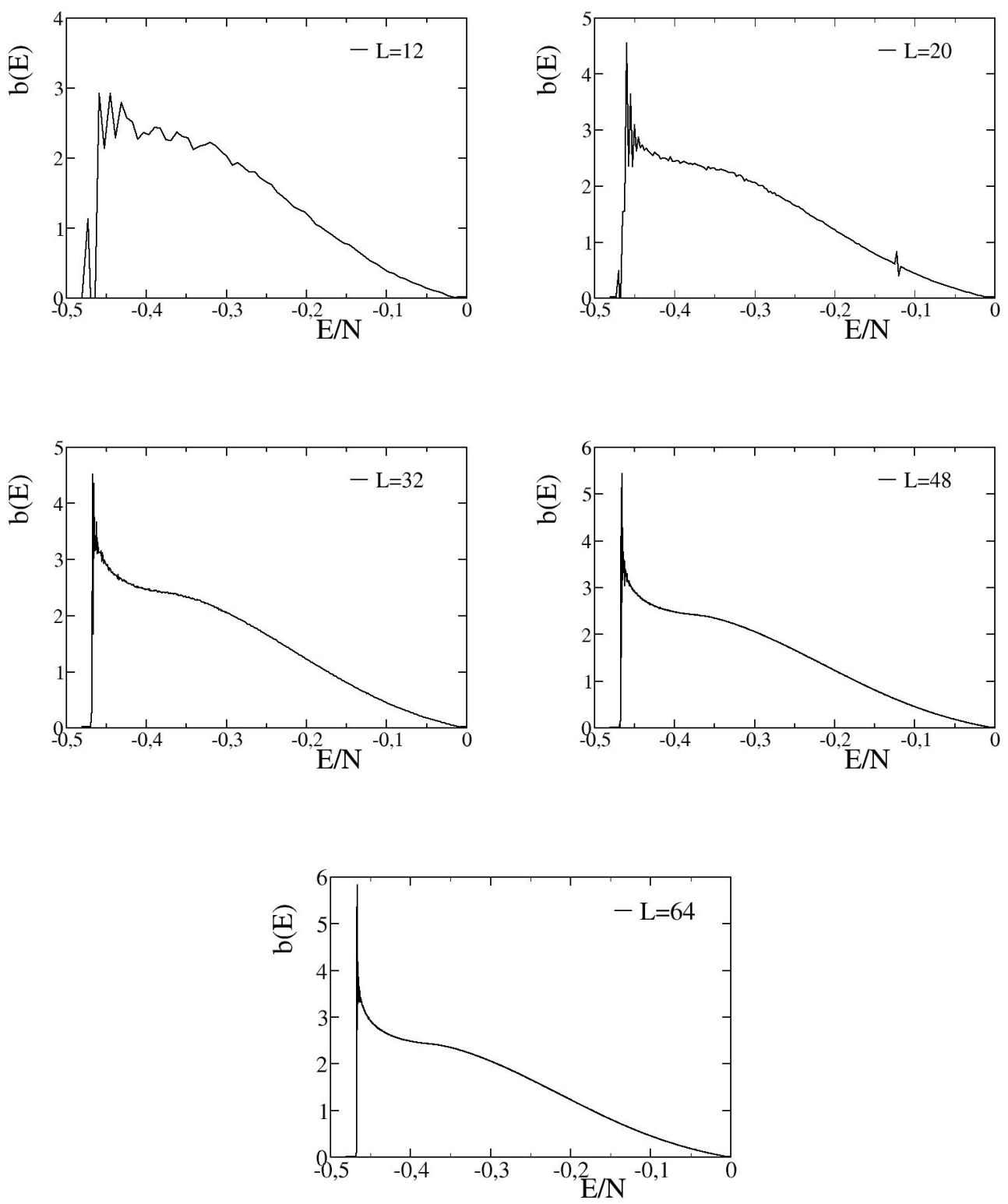

Figura A.6: Parâmetros multicanônicos $b(E)$ finais utilizados para a produção de dados para $\delta=0.93$. 

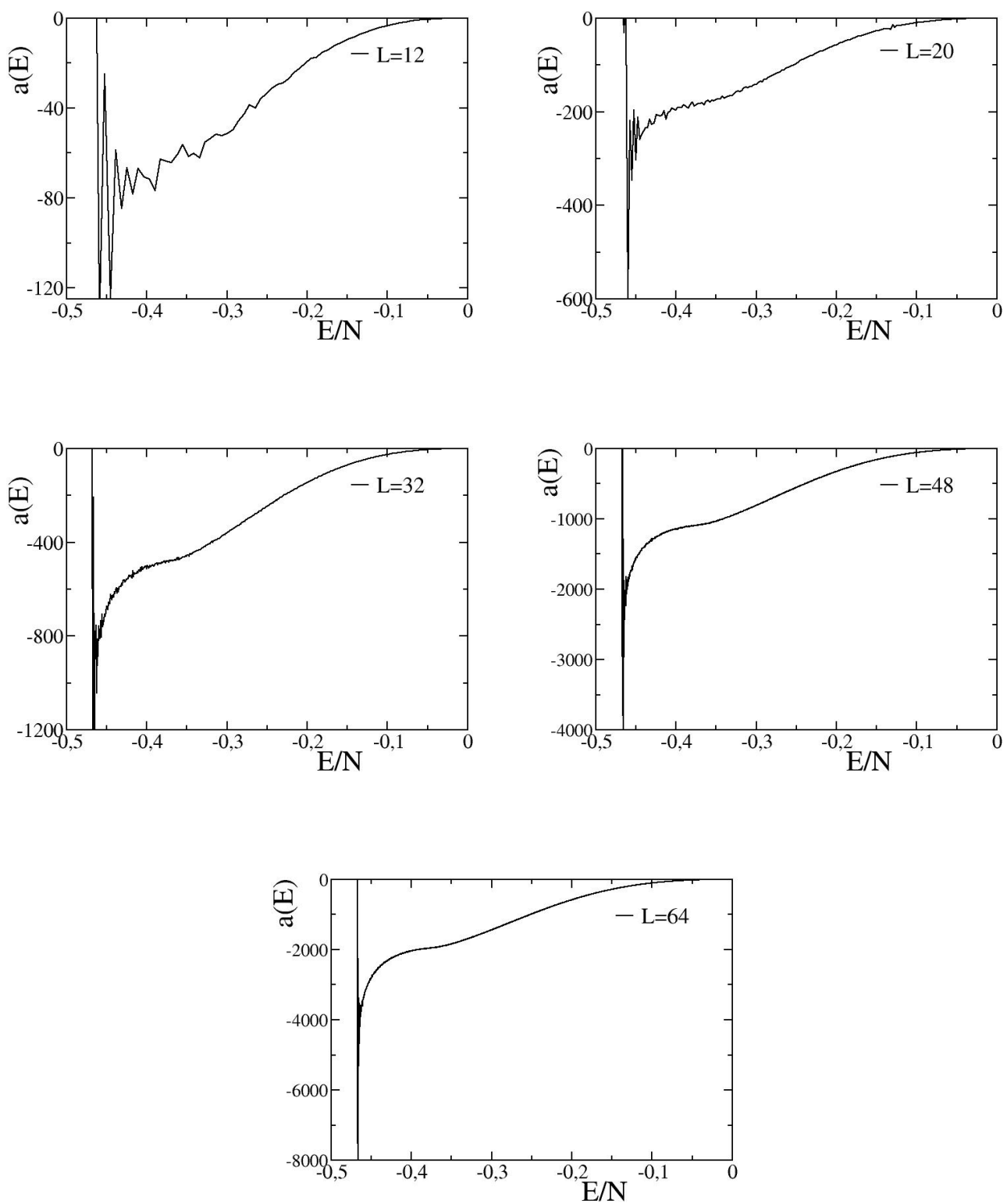

Figura A.7: Parâmetros multicanônicos $a(E)$ finais utilizados para a produção de dados para $\delta=0.95$. 

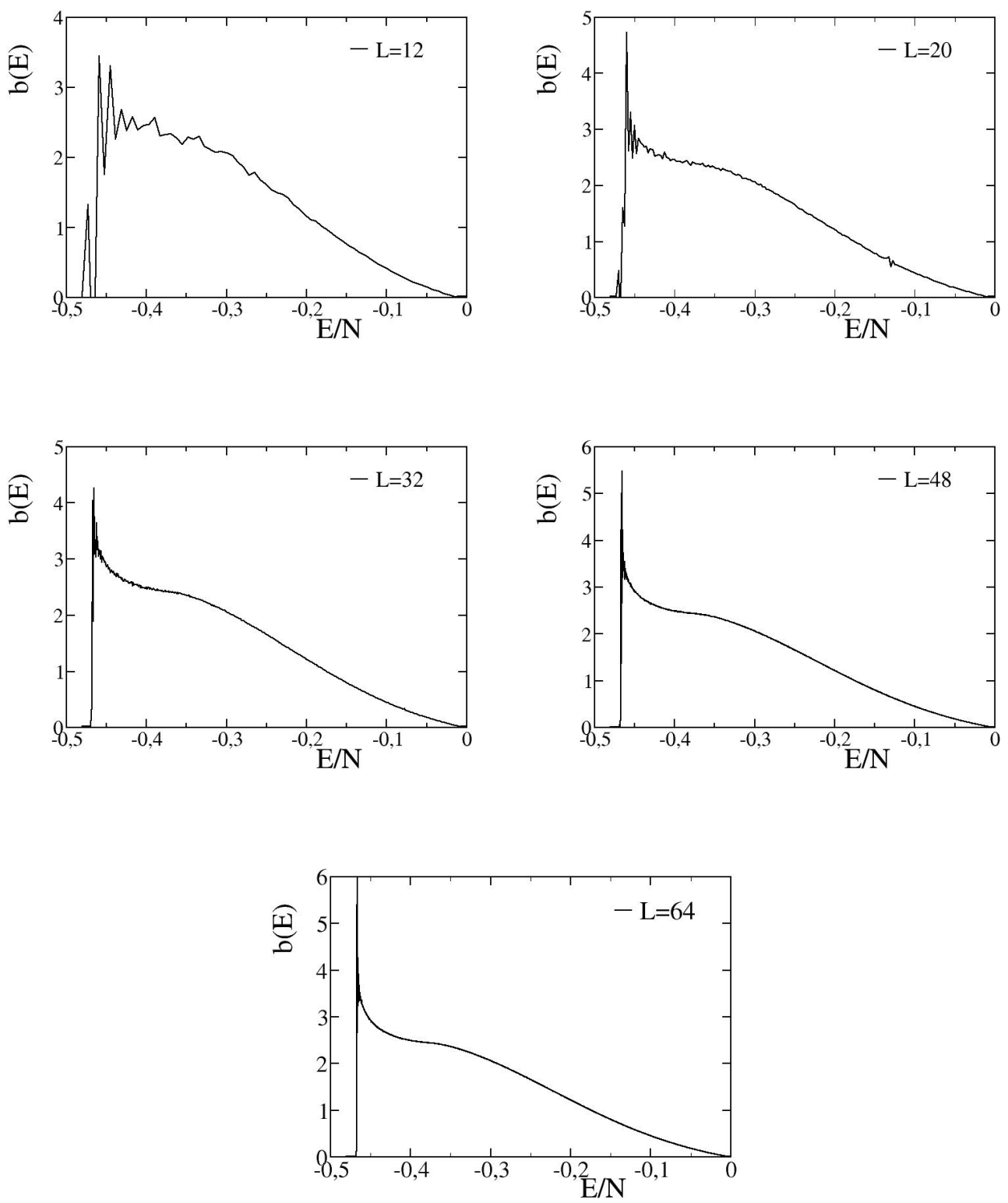

Figura A.8: Parâmetros multicanônicos $b(E)$ finais utilizados para a produção de dados para $\delta=0.95$. 

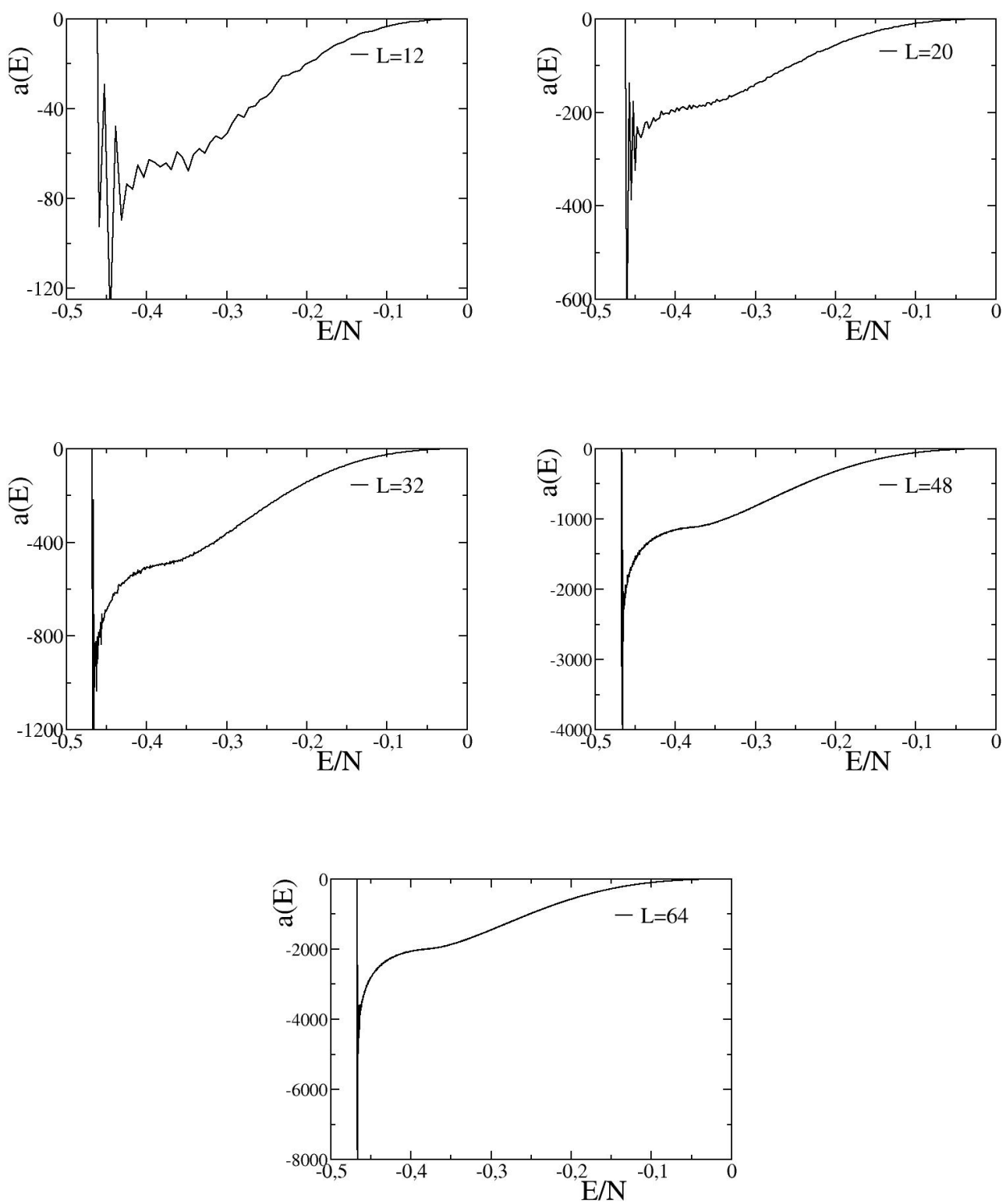

Figura A.9: Parâmetros multicanônicos $a(E)$ finais utilizados para a produção de dados para $\delta=0.97$. 

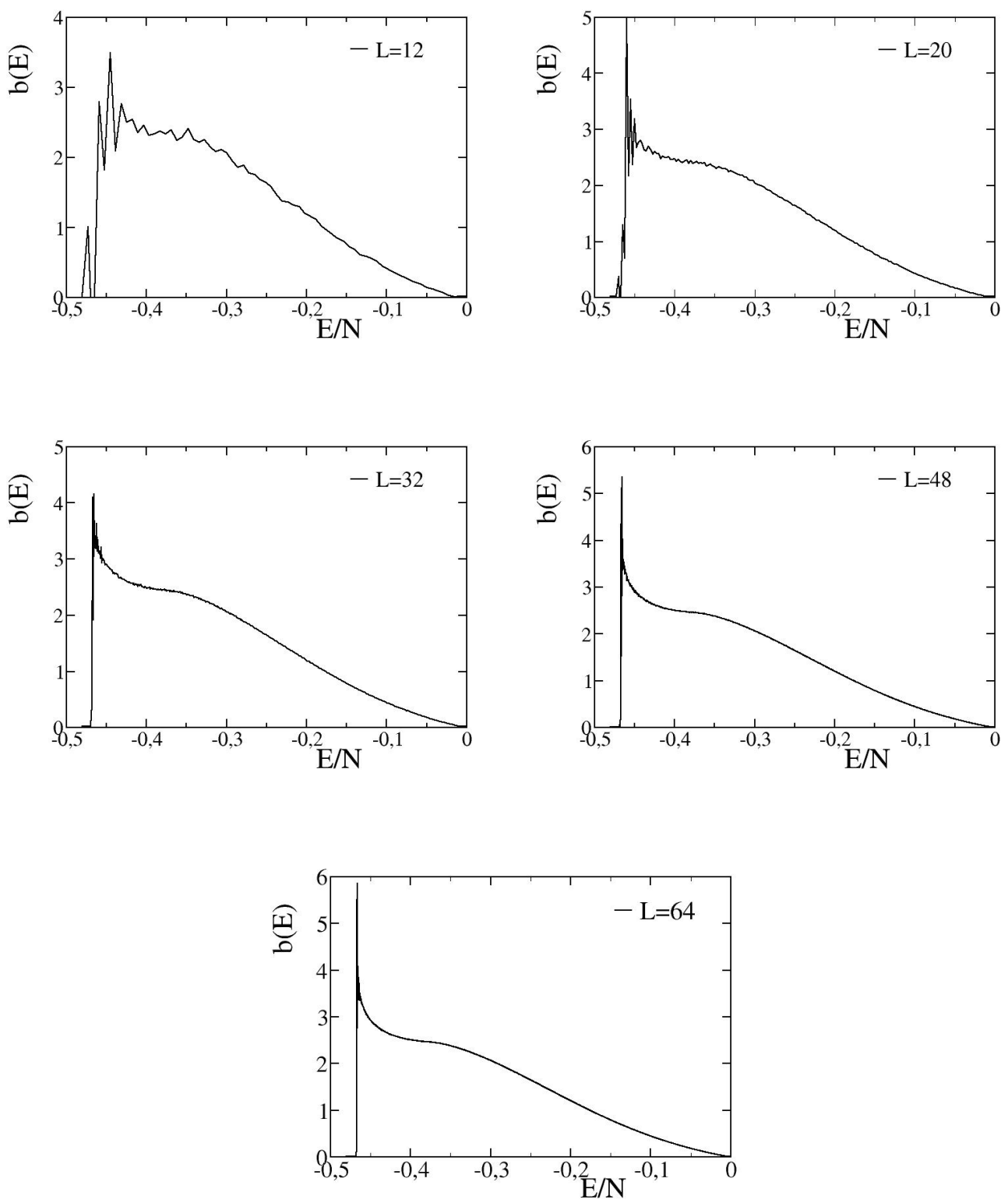

Figura A.10: Parâmetros multicanônicos $b(E)$ finais utilizados para a produção de dados para $\delta=0.97$. 

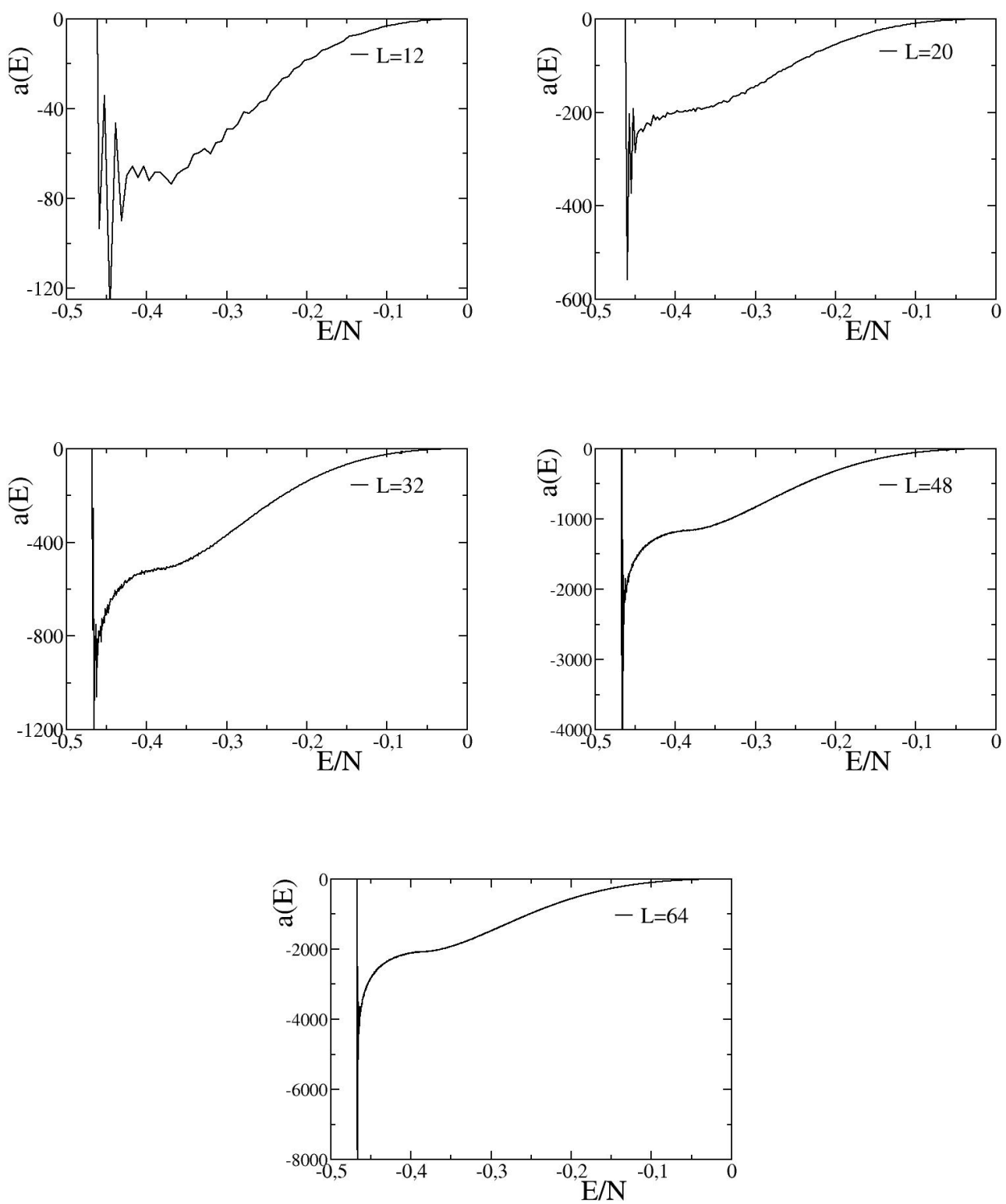

Figura A.11: Parâmetros multicanônicos $a(E)$ finais utilizados para a produção de dados para $\delta=1.00$. 

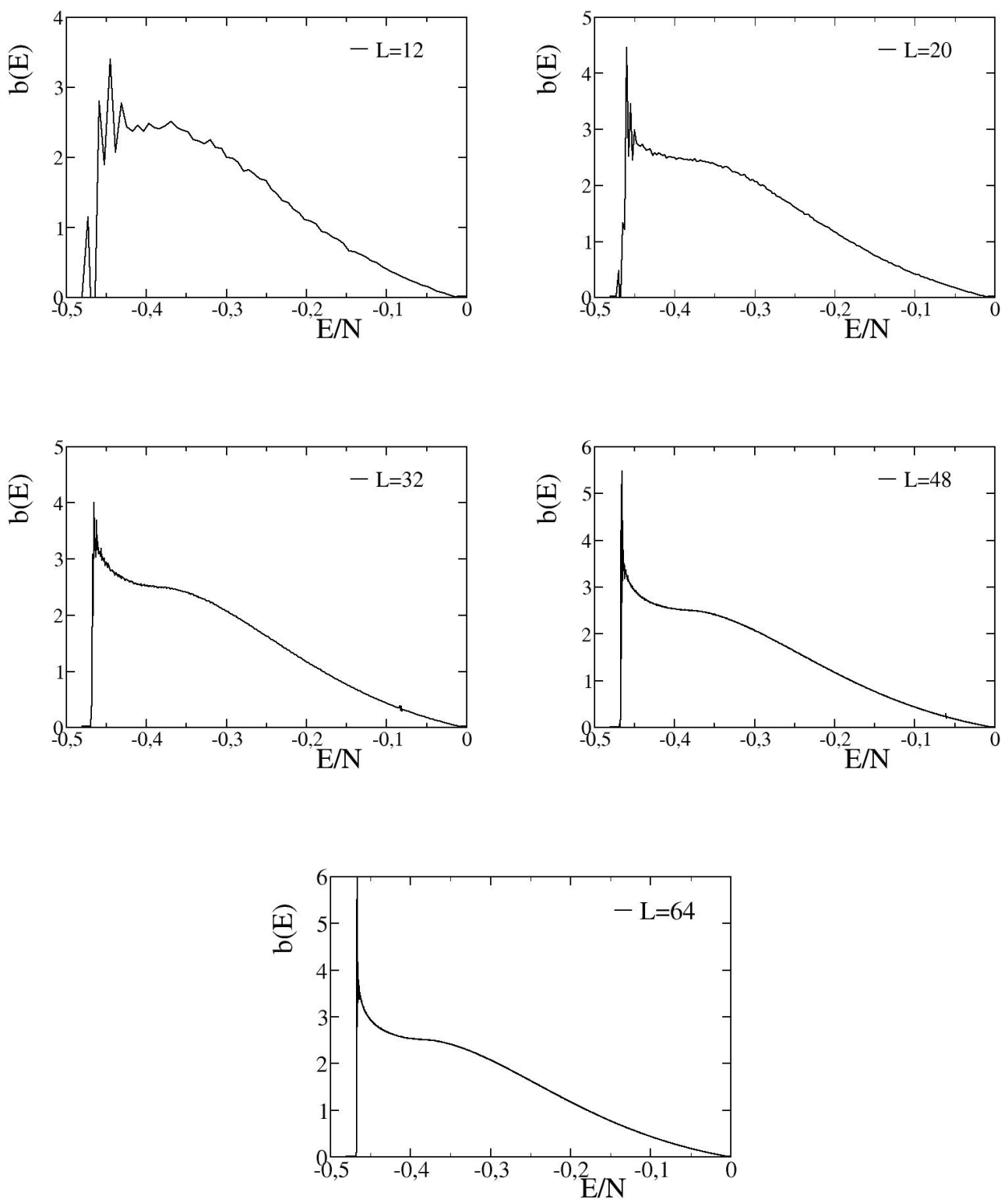

Figura A.12: Parâmetros multicanônicos $b(E)$ finais utilizados para a produção de dados para $\delta=1.00$. 

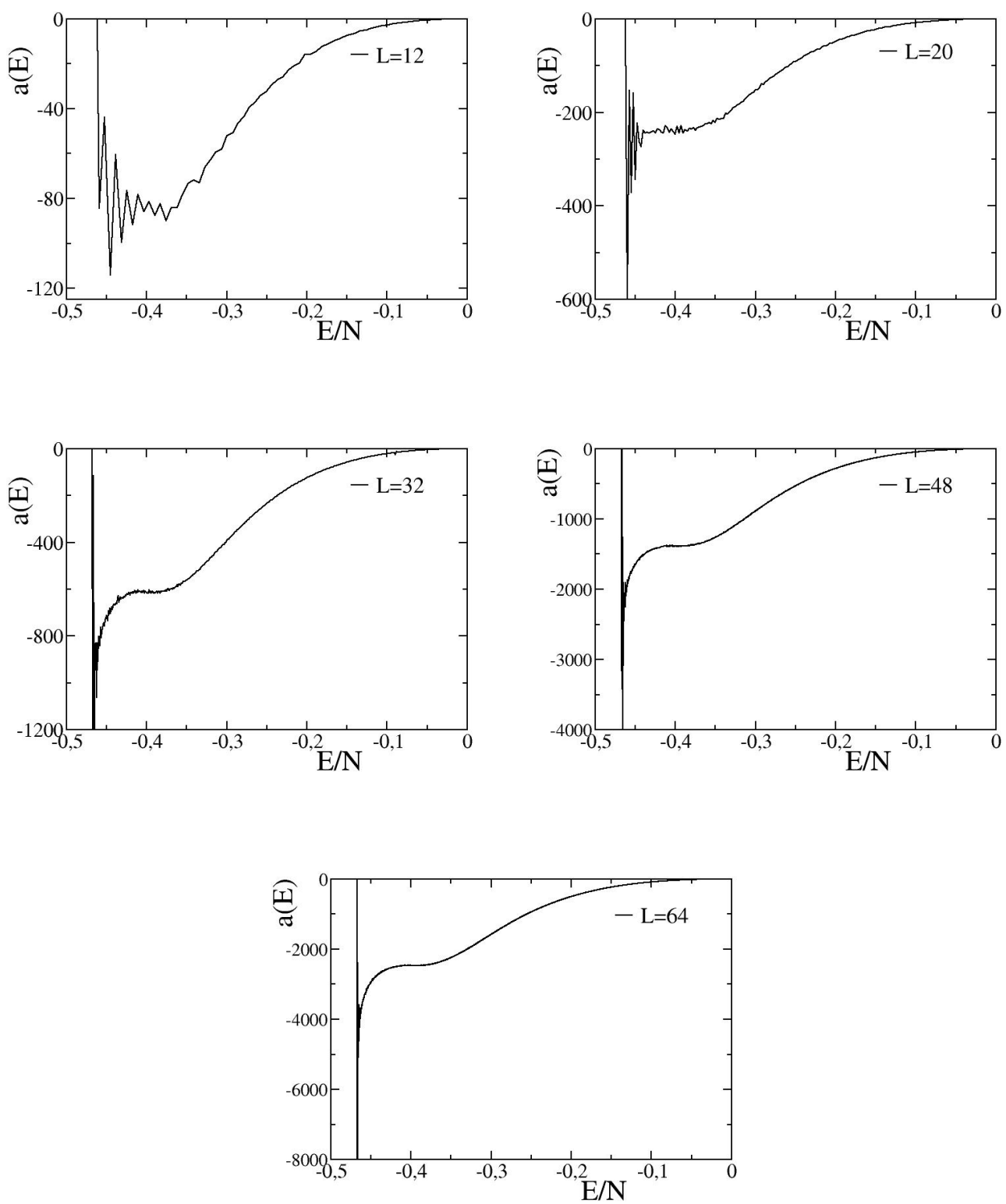

Figura A.13: Parâmetros multicanônicos $a(E)$ finais utilizados para a produção de dados para $\delta=1.10$. 

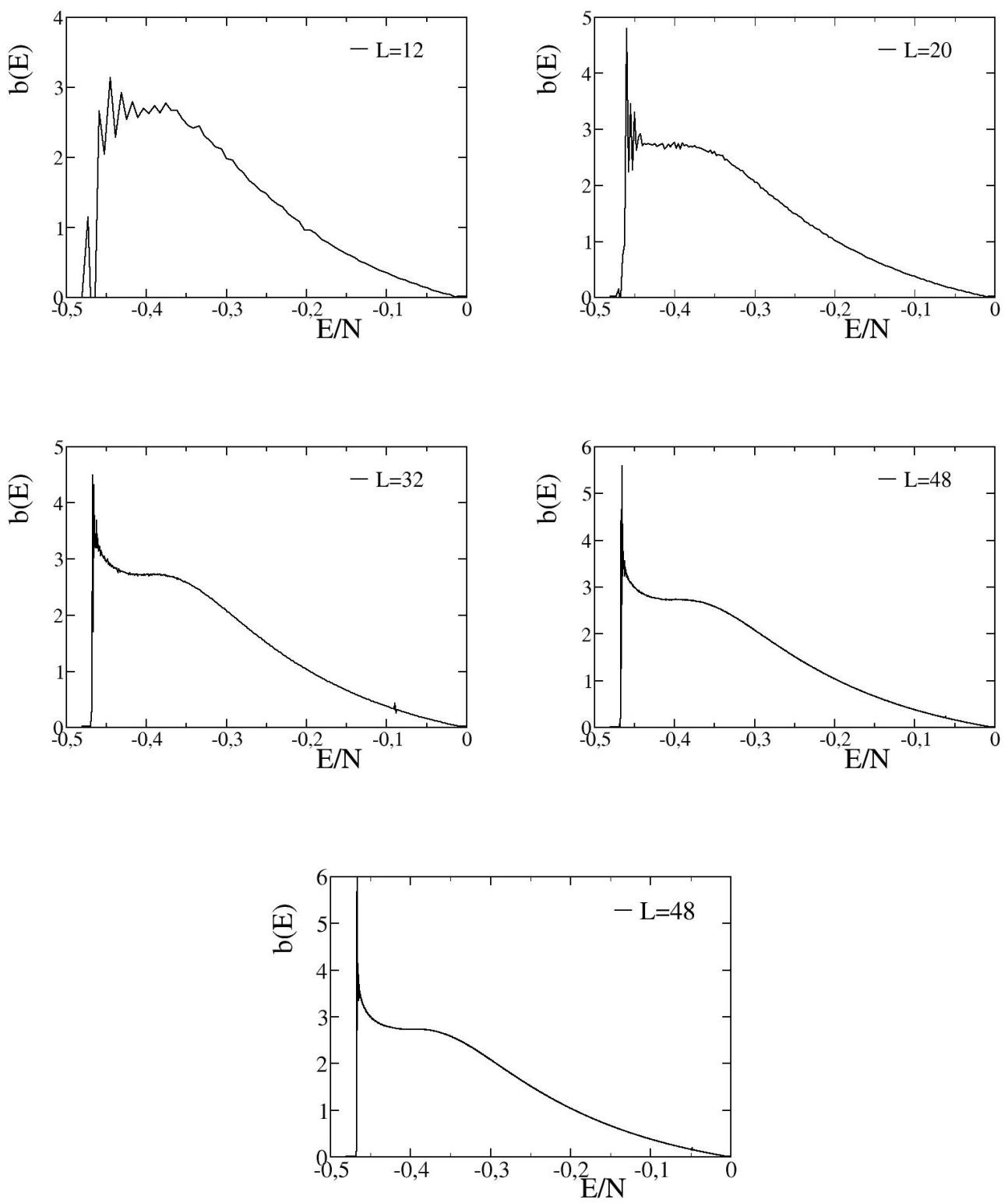

Figura A.14: Parâmetros multicanônicos $b(E)$ finais utilizados para a produção de dados para $\delta=1.10$. 

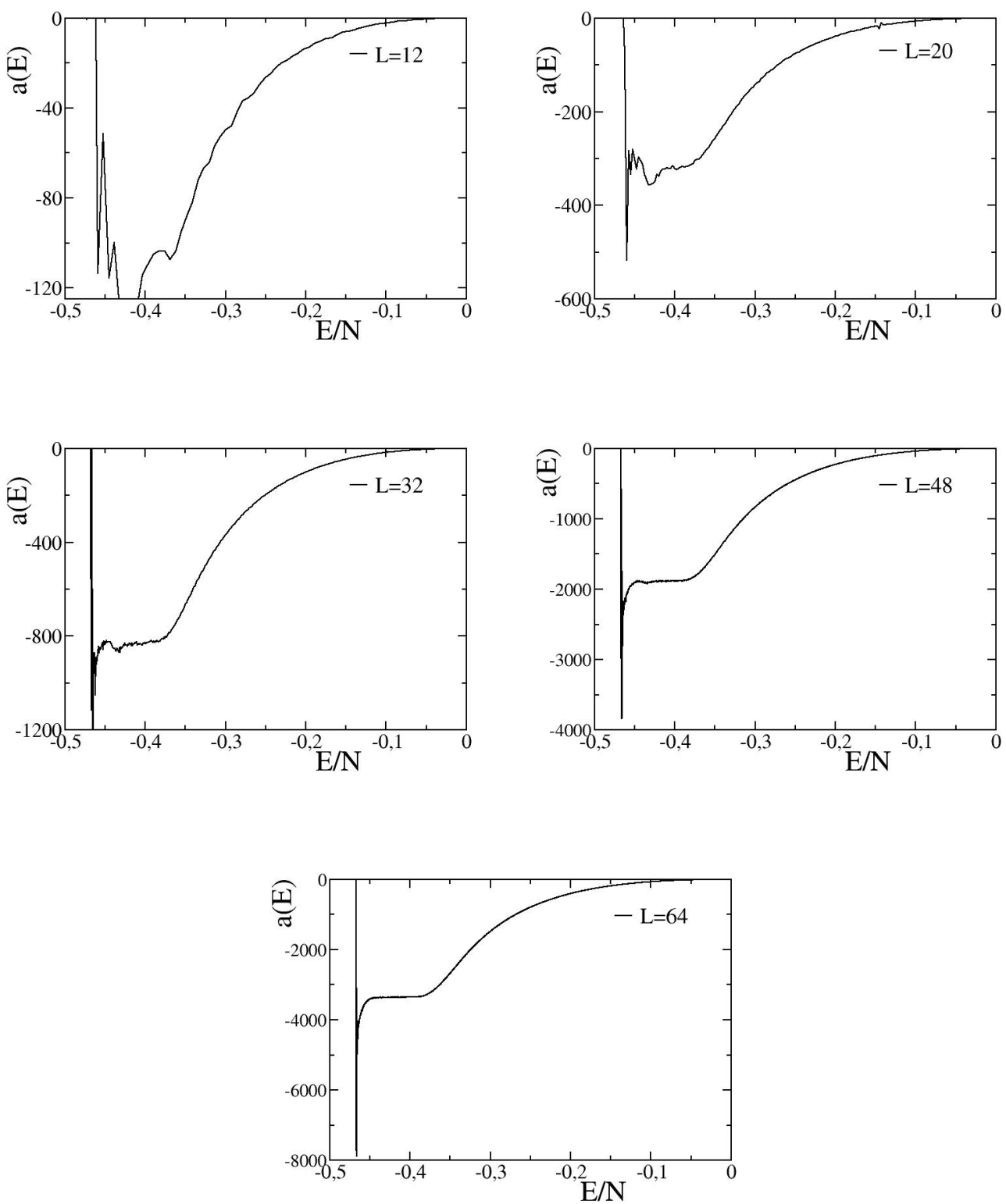

Figura A.15: Parâmetros multicanônicos $a(E)$ finais utilizados para a produção de dados para $\delta=1.20$. 

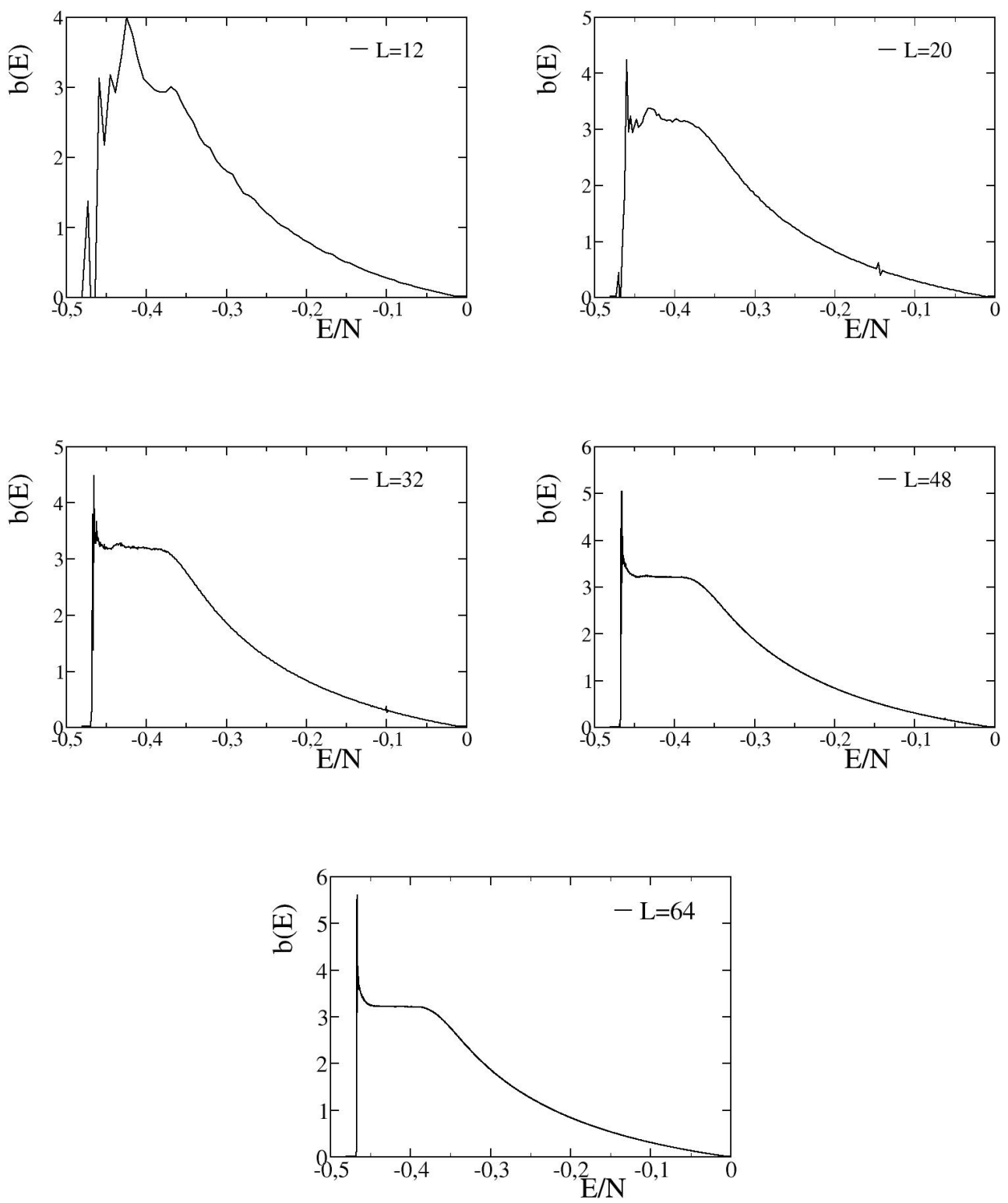

Figura A.16: Parâmetros multicanônicos $b(E)$ finais utilizados para a produção de dados para $\delta=1.20$. 

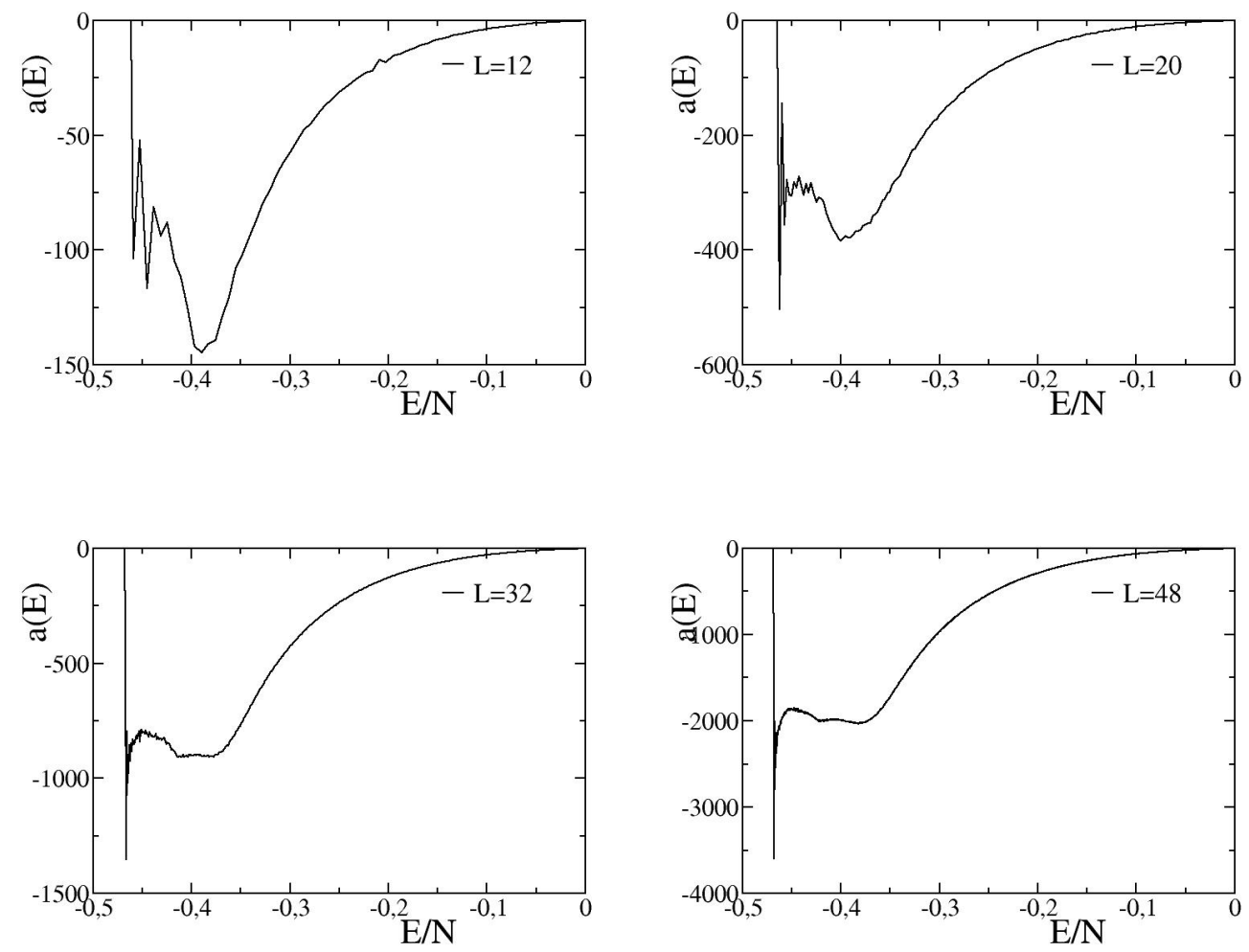

Figura A.17: Parâmetros multicanônicos $a(E)$ finais utilizados para a produção de dados para $\delta=1.30$. 

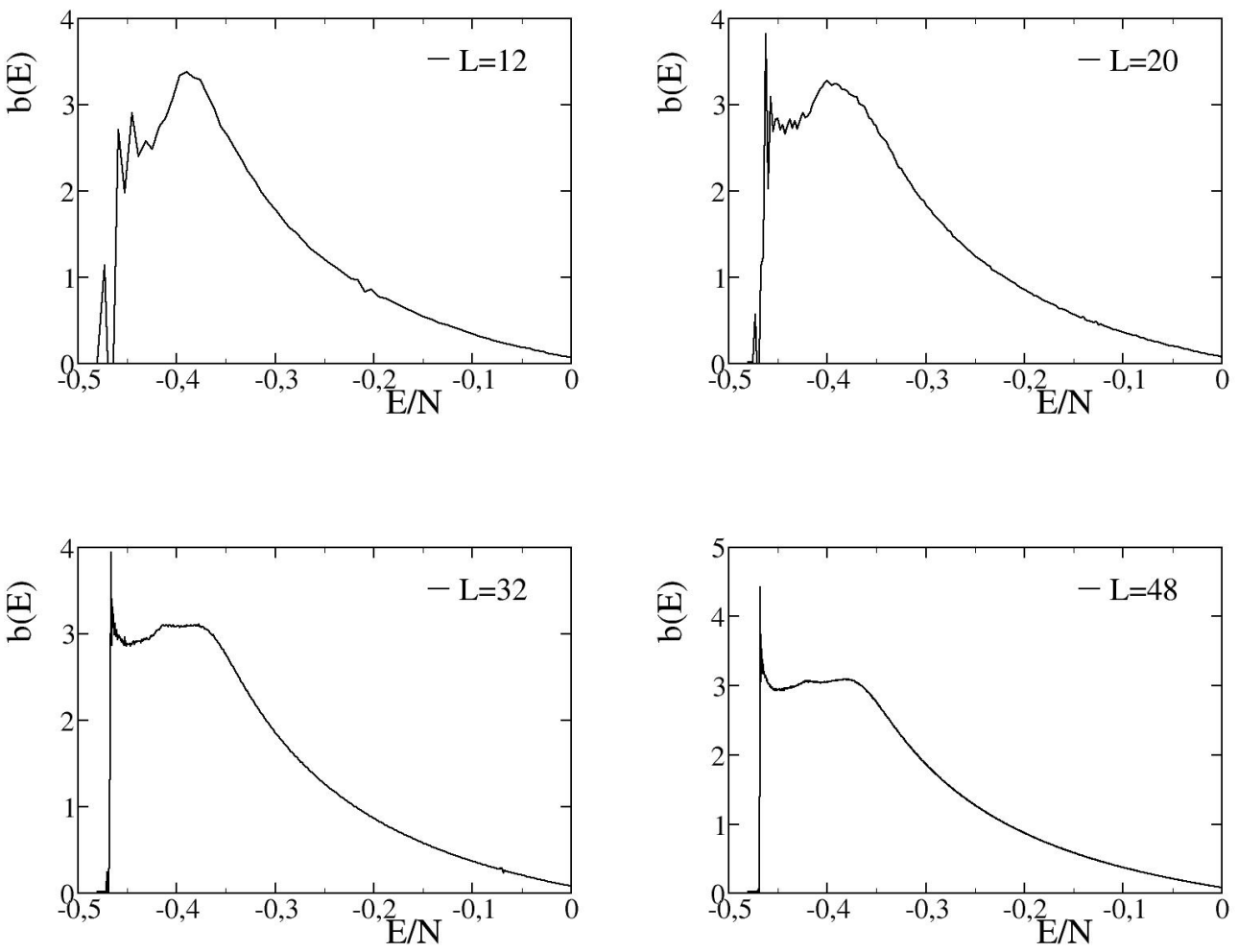

Figura A.18: Parâmetros multicanônicos $b(E)$ finais utilizados para a produção de dados para $\delta=1.30$. 


\section{APÊNDICE}

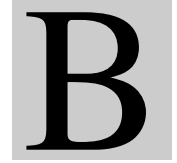

\section{ZEROS COMPLEXOS DA FUNÇÃO DE PARTIÇÃO}

Neste apêndice incluimos as tabelas referentes ao primeiro zero complexo da função de partição para todos os valores de $\delta$ e $L$ utilizados em nossas simulações. Nestas tabelas, os números entre parênteses correspondem ao erro estatístico obtido com as 16 séries temporais. 


\begin{tabular}{|l|l|l|l|l|}
\hline \multicolumn{5}{|c|}{$\delta=0.89$} \\
\hline$L$ & $\operatorname{Re}\left(u_{1}^{0}\right)$ & $\operatorname{Im}\left(u_{1}^{0}\right)$ & $\operatorname{Re}\left(\beta_{1}^{0}\right)$ & $\operatorname{Im}\left(\beta_{1}^{0}\right)$ \\
\hline 12 & $0.091(4)$ & $0.040(3)$ & $2.31(3)$ & $0.41(3)$ \\
20 & $0.091(2)$ & $0.022(1)$ & $2.37(2)$ & $0.24(2)$ \\
32 & $0.091(1)$ & $0.0133(5)$ & $2.39(1)$ & $0.145(6)$ \\
48 & $0.0903(7)$ & $0.0084(6)$ & $2.400(8)$ & $0.093(6)$ \\
64 & $0.089(1)$ & $0.0061(4)$ & $2.41(1)$ & $0.069(4)$ \\
\hline
\end{tabular}

Tabela B.1: Zeros para $\delta=0.89$.

\begin{tabular}{|l|l|l|l|l|}
\hline \multicolumn{5}{|c|}{$\delta=0.91$} \\
\hline$L$ & $\operatorname{Re}\left(u_{1}^{0}\right)$ & $\operatorname{Im}\left(u_{1}^{0}\right)$ & $\operatorname{Re}\left(\beta_{1}^{0}\right)$ & $\operatorname{Im}\left(\beta_{1}^{0}\right)$ \\
\hline 12 & $0.092(4)$ & $0.038(3)$ & $2.30(4)$ & $0.39(3)$ \\
20 & $0.091(2)$ & $0.0212(9)$ & $2.37(3)$ & $0.23(1)$ \\
32 & $0.090(1)$ & $0.0130(9)$ & $2.40(1)$ & $0.14(1)$ \\
48 & $0.089(1)$ & $0.0081(5)$ & $2.42(1)$ & $0.091(6)$ \\
64 & $0.0886(6)$ & $0.0060(3)$ & $2.421(7)$ & $0.067(4)$ \\
\hline
\end{tabular}

Tabela B.2: Zeros para $\delta=0.91$.

\begin{tabular}{|r|l|l|l|l|}
\hline \multicolumn{5}{|c|}{$\delta=0.93$} \\
\hline$L$ & $\operatorname{Re}\left(u_{1}^{0}\right)$ & $\operatorname{Im}\left(u_{1}^{0}\right)$ & $\operatorname{Re}\left(\beta_{1}^{0}\right)$ & $\operatorname{Im}\left(\beta_{1}^{0}\right)$ \\
\hline 12 & $0.091(3)$ & $0.038(2)$ & $2.32(3)$ & $0.39(2)$ \\
20 & $0.090(1)$ & $0.020(1)$ & $2.38(2)$ & $0.22(1)$ \\
32 & $0.089(1)$ & $0.0124(6)$ & $2.41(1)$ & $0.139(7)$ \\
48 & $0.0878(5)$ & $0.0077(5)$ & $2.430(6)$ & $0.087(5)$ \\
64 & $0.0873(5)$ & $0.0057(4)$ & $2.436(6)$ & $0.065(4)$ \\
\hline
\end{tabular}

Tabela B.3: Zeros para $\delta=0.93$. 


\begin{tabular}{|l|l|l|l|l|}
\hline \multicolumn{5}{|c|}{$\delta=0.95$} \\
\hline$L$ & $\operatorname{Re}\left(u_{1}^{0}\right)$ & $\operatorname{Im}\left(u_{1}^{0}\right)$ & $\operatorname{Re}\left(\beta_{1}^{0}\right)$ & $\operatorname{Im}\left(\beta_{1}^{0}\right)$ \\
\hline 12 & $0.089(3)$ & $0.036(2)$ & $2.34(3)$ & $0.38(3)$ \\
20 & $0.089(1)$ & $0.019(1)$ & $2.40(0)$ & $0.22(1)$ \\
32 & $0.0871(8)$ & $0.0116(8)$ & $2.43(1)$ & $0.132(8)$ \\
48 & $0.0863(5)$ & $0.0073(3)$ & $2.446(6)$ & $0.084(4)$ \\
64 & $0.0858(3)$ & $0.0051(2)$ & $2.454(3)$ & $0.060(2)$ \\
\hline
\end{tabular}

Tabela B.4: Zeros para $\delta=0.95$.

\begin{tabular}{|l|l|l|l|l|}
\hline \multicolumn{5}{|c|}{$\delta=0.97$} \\
\hline$L$ & $\operatorname{Re}\left(u_{1}^{0}\right)$ & $\operatorname{Im}\left(u_{1}^{0}\right)$ & $\operatorname{Re}\left(\beta_{1}^{0}\right)$ & $\operatorname{Im}\left(\beta_{1}^{0}\right)$ \\
\hline 12 & $0.087(2)$ & $0.034(2)$ & $2.37(2)$ & $0.37(2)$ \\
20 & $0.086(1)$ & $0.0184(7)$ & $2.43(1)$ & $0.211(8)$ \\
32 & $0.0850(6)$ & $0.0105(5)$ & $2.458(6)$ & $0.123(6)$ \\
48 & $0.0845(4)$ & $0.0068(4)$ & $2.468(5)$ & $0.080(5)$ \\
64 & $0.0841(3)$ & $0.0048(3)$ & $2.474(3)$ & $0.057(3)$ \\
\hline
\end{tabular}

Tabela B.5: Zeros para $\delta=0.97$.

\begin{tabular}{|r|l|l|l|l|}
\hline \multicolumn{5}{|c|}{$\delta=1.00$} \\
\hline$L$ & $\operatorname{Re}\left(u_{1}^{0}\right)$ & $\operatorname{Im}\left(u_{1}^{0}\right)$ & $\operatorname{Re}\left(\beta_{1}^{0}\right)$ & $\operatorname{Im}\left(\beta_{1}^{0}\right)$ \\
\hline 12 & $0.084(2)$ & $0.031(2)$ & $2.41(2)$ & $0.35(2)$ \\
20 & $0.0830(9)$ & $0.0164(4)$ & $2.47(1)$ & $0.195(4)$ \\
32 & $0.0816(6)$ & $0.0092(4)$ & $2.499(8)$ & $0.112(5)$ \\
48 & $0.0812(4)$ & $0.0058(2)$ & $2.508(5)$ & $0.071(3)$ \\
64 & $0.0810(2)$ & $0.0041(2)$ & $2.513(2)$ & $0.050(3)$ \\
\hline
\end{tabular}

Tabela B.6: Zeros para $\delta=1.00$. 


\begin{tabular}{|l|l|l|l|l|}
\hline \multicolumn{5}{|c|}{$\delta=1.10$} \\
\hline$L$ & $\operatorname{Re}\left(u_{1}^{0}\right)$ & $\operatorname{Im}\left(u_{1}^{0}\right)$ & $\operatorname{Re}\left(\beta_{1}^{0}\right)$ & $\operatorname{Im}\left(\beta_{1}^{0}\right)$ \\
\hline 12 & $0.067(1)$ & $0.0222(9)$ & $2.66(2)$ & $0.322(9)$ \\
20 & $0.0658(6)$ & $0.0105(3)$ & $2.71(1)$ & $0.158(4)$ \\
32 & $0.0652(5)$ & $0.0054(2)$ & $2.726(7)$ & $0.083(3)$ \\
48 & $0.0650(2)$ & $0.0031(1)$ & $2.732(3)$ & $0.048(2)$ \\
64 & $0.06488(9)$ & $0.00207(8)$ & $2.735(1)$ & $0.032(1)$ \\
\hline
\end{tabular}

Tabela B.7: Zeros para $\delta=1.10$.

\begin{tabular}{|l|l|l|l|l|}
\hline \multicolumn{5}{|c|}{$\delta=1.20$} \\
\hline$L$ & $\operatorname{Re}\left(u_{1}^{0}\right)$ & $\operatorname{Im}\left(u_{1}^{0}\right)$ & $\operatorname{Re}\left(\beta_{1}^{0}\right)$ & $\operatorname{Im}\left(\beta_{1}^{0}\right)$ \\
\hline 12 & $0.036(1)$ & $0.0150(5)$ & $3.23(4)$ & $0.391(7)$ \\
20 & $0.0397(8)$ & $0.0069(3)$ & $3.21(2)$ & $0.17(1)$ \\
32 & $0.0400(2)$ & $0.0033(2)$ & $3.214(5)$ & $0.081(4)$ \\
48 & $0.04005(9)$ & $0.00158(9)$ & $3.217(2)$ & $0.040(2)$ \\
64 & $0.03994(6)$ & $0.00097(5)$ & $3.220(1)$ & $0.024(1)$ \\
\hline
\end{tabular}

Tabela B.8: Zeros para $\delta=1.20$.

\begin{tabular}{|l|l|l|l|l|}
\hline \multicolumn{5}{|c|}{$\delta=1.30$} \\
\hline$L$ & $\operatorname{Re}\left(u_{1}^{0}\right)$ & $\operatorname{Im}\left(u_{1}^{0}\right)$ & $\operatorname{Re}\left(\beta_{1}^{0}\right)$ & $\operatorname{Im}\left(\beta_{1}^{0}\right)$ \\
\hline 12 & $0.051(1)$ & $0.0133(3)$ & $2.93(2)$ & $0.253(2)$ \\
20 & $0.0509(5)$ & $0.00420(3)$ & $2.974(9)$ & $0.0824(4)$ \\
32 & $0.0488(1)$ & $0.00175(1)$ & $3.020(3)$ & $0.0358(3)$ \\
48 & $0.04810(7)$ & $0.000778(5)$ & $3.034(1)$ & $0.0162(1)$ \\
\hline
\end{tabular}

Tabela B.9: Zeros para $\delta=1.30$. 


\section{APÊNDICE}

\section{ANÁLISE MICROCANÔNICA}

Como complemento a esse trabalho, utilizamos a abordagem microcanônica com o intuito de verificar as ordens da transição de fase e a existência de outras fases diferentes da fase tipo faixas e tetragonal. Essa análise foi feita para redes de tamanho $L=64$, já que essa análise pode ser feita para redes finitas sem necessitar de uma análise de escala de tamanho finito. Para sistemas finitos, a análise microcanônica apresenta calor específico negativo para transições de fase de primeira ordem. Esse efeito se deve a uma concavidade na entropia microcanônica em relação a $e_{s}=E / N$. A entropia microcanônica é obtida diretamente da densidade de estados. Aqui, a densidade de estados sai diretamente dos pesos multicanônicos, já que, neste ensemble, a entropia $S$ é dada por:

$$
S=-b(E) E+a(E)
$$

onde $b(E)$, já mencionado anteriomente, está relacionado à temperatura microcanônica por $b(E)=1 / T$. Então, da eq. C.1, temos que $b(E)=(\partial S / \partial E)$, ou $T(E)=(\partial S / \partial E)^{-1}$. Assim, o calor específico a volume constante, fica escrito como:

$$
C_{v}=\partial E / \partial T(E)=-(\partial S / \partial E)^{2} /\left(\partial^{2} S / \partial^{2} E^{2}\right) .
$$

Escrevendo o calor específico em função de $b(E)$, temos:

$$
C_{v}=-b(E)^{2} /\left(\frac{\partial b(E)}{\partial E}\right) .
$$

Olhando para a equação C.3, observamos que um ponto de inflexão no gráfico de $b(E)$ versus $E / N$ leva a derivadas positivas de $b(E)$ e, consequentemente, à valores negativos para $C_{v}$. 
Encontramos nessa análise indícios de mais de uma fase para $\delta=1.20$ e 1.30, colocando em dúvida se o ponto tricrítico se localiza na linha de transição da fase do tipo faixas para a fase tetragonal ou na linha de transição entre a fase nemática e a tetragonal. As figuras C.1 e C.2, contém os gráficos de $C_{v}$ versus $E / N$ para os valores de $\delta$ simulados de 0.89 a 1.10 . Em ambas as figuras observamos somente uma transição de segunda ordem, já que temos picos para o calor específico e valores positivos.

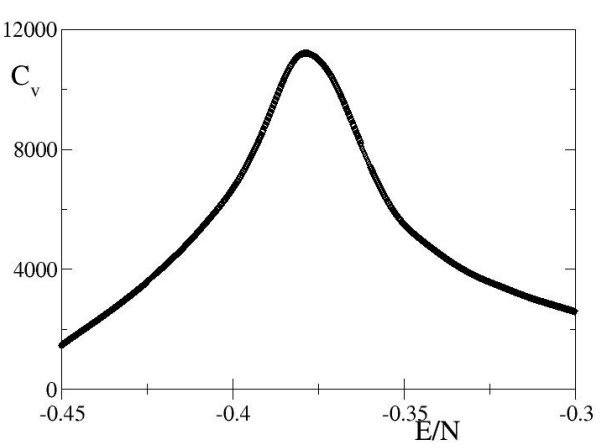

(a)

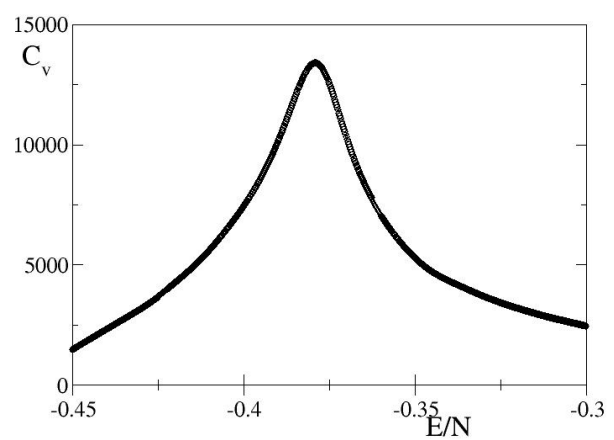

(c)

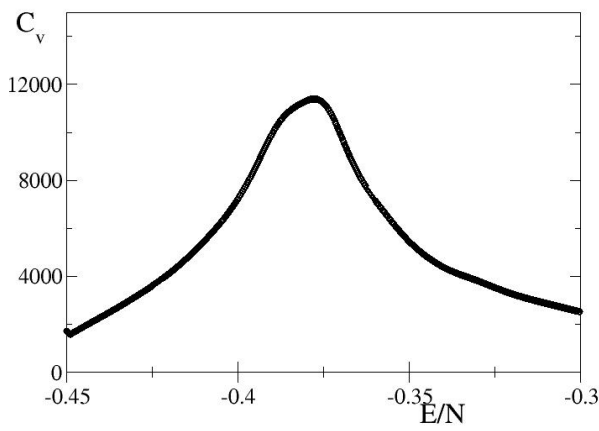

(b)

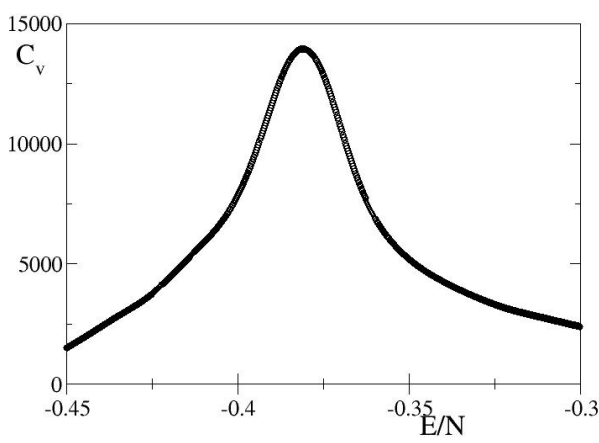

(d)

Figura C.1: Calores específicos microcanônicos para (a) $\delta=0.89$ (b) $\delta=0.91$, (c) $\delta=0.93,(\mathrm{~d}) \delta=0.95$. 


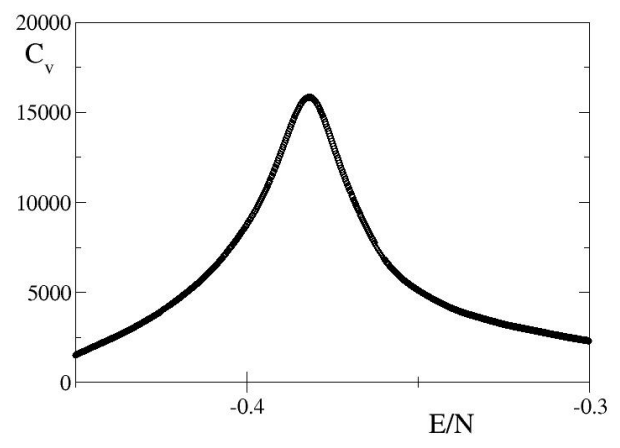

(a)

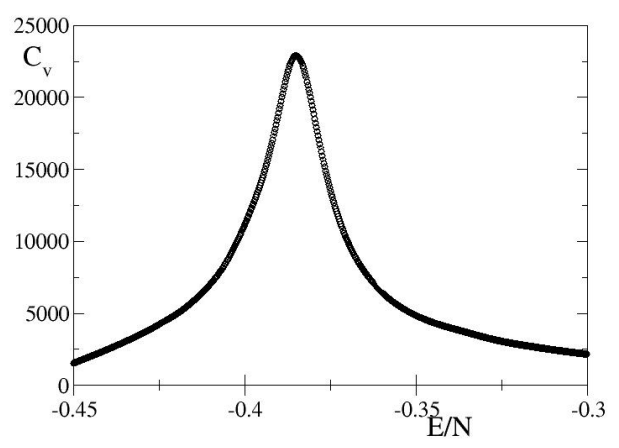

(b)

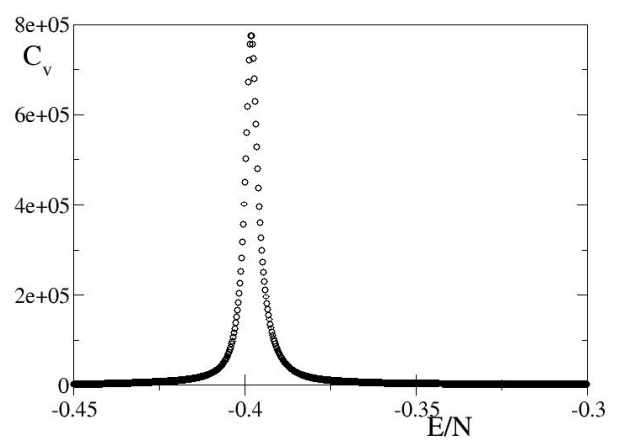

(c)

Figura C.2: Calores específicos microcanônicos para (a) $\delta=0.97$ (b) $\delta=1.00$, (c) $\delta=1.10$. 


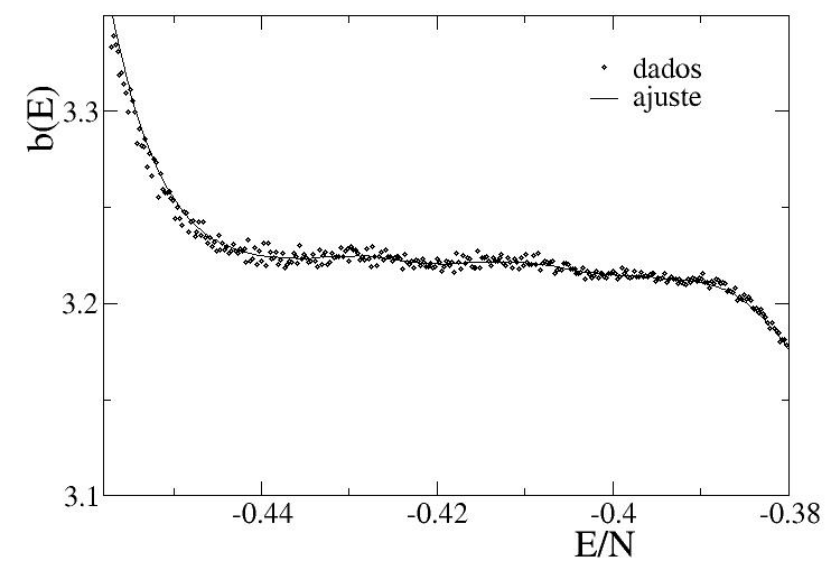

(a)

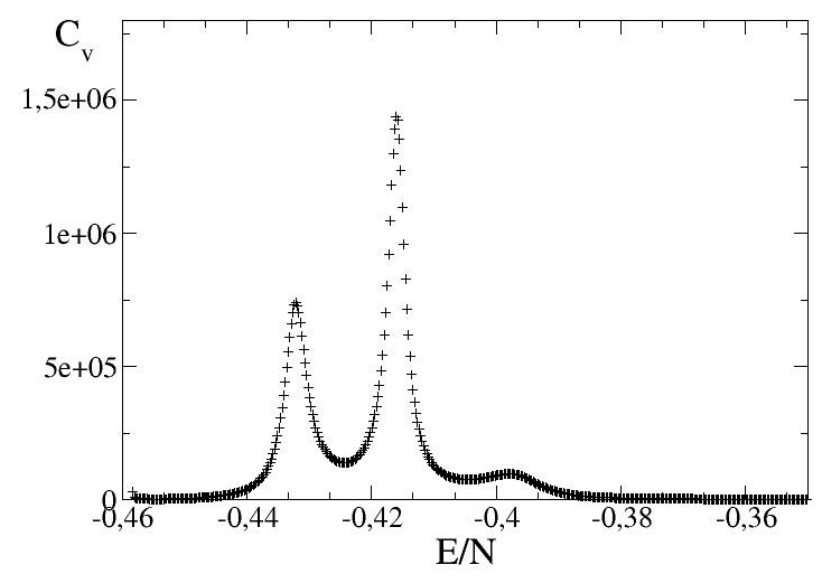

(b)

Figura C.3: $\delta=1.20($ a) $b(E)$ versus $E / N$, (b) calor específico microcanônico. 


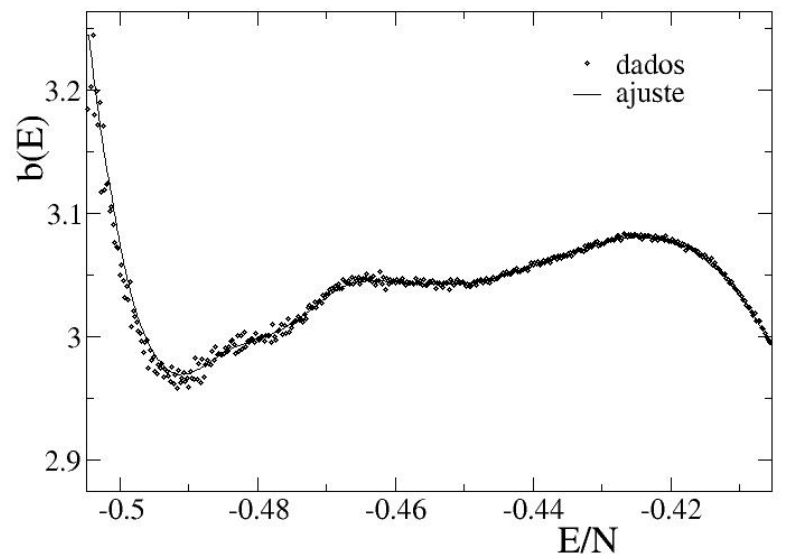

(a)

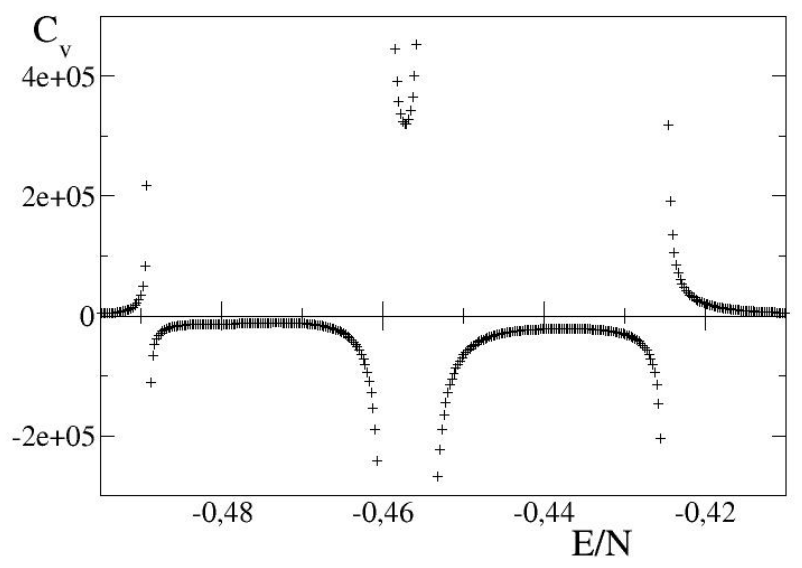

(b)

Figura C.4: $\delta=1.30($ a) $b(E)$ versus $E / N$, (b) calor específico microcanônico. 